Marquette University

e-Publications@Marquette

Mathematics, Statistics and Computer Science

Mathematics, Statistics and Computer Science,

Faculty Research and Publications

Department of

$1-1-2008$

\title{
Molecular Targets of 2,3,7,8-tetrachlorodibenzo-p- dioxin (TCDD) within the Zebrafish Ovary: Insights into TCDD-induced Endocrine Disruption and Reproductive Toxicity
}

Tisha C. King Heiden

University of Wisconsin - Milwaukee

Craig Struble

Marquette University, craig.struble@marquette.edu

Matthew L. Rise

University of Wisconsin - Milwaukee

Martin J. Hessner

Medical College of Wisconsin

Reinhold J. Hutz

University of Wisconsin - Milwaukee

See next page for additional authors

Accepted version. Reproductive Toxicology, Vol. 25, No. 1 (January 2008): 47-57. DOI. (C) 2008

Elsevier. Used with permission. 
Authors

Tisha C. King Heiden, Craig Struble, Matthew L. Rise, Martin J. Hessner, Reinhold J. Hutz, and Michael J. Carvan III 


\title{
Molecular Targets of 2,3,7,8- Tetrachlorodibenzo-P-Dioxin (TCDD) Within The Zebrafish Ovary: Insights into TCDD-Induced Endocrine Disruption and Reproductive Toxicity
}

\author{
Tisha C. King Heiden \\ Department of Biological Sciences, \\ Marine \& Freshwater Biomedical Sciences Center, \\ Great Lakes WATER Institute, University of Wisconsin-Milwaukee \\ Milwaukee, WI \\ Craig A. Struble \\ Department of Mathematics, Marquette University \\ Milwaukee, WI \\ Matthew L. Rise \\ Great Lakes WATER Institute, University of Wisconsin-Milwaukee \\ Milwaukee, WI \\ Martin J. Hessner \\ Department of Pediatrics, Medical College of Wisconsin \\ Milwaukee, WI
}

Reproductive Toxicology, Vol. 25, No. 1 (January 2008): pg. 47-57. DOI. This article is @ Elsevier and permission has been granted for this version to appear in e-Publications@Marquette. Elsevier does not grant permission for this article to be further copied/distributed or hosted elsewhere without the express permission from Elsevier. 


\author{
Reinhold J. Hutz \\ Department of Biological Sciences, \\ Marine \& Freshwater Biomedical Sciences Center, \\ University of Wisconsin-Milwaukee \\ Milwaukee, WI \\ Michael J. Carvan, III \\ Marine \& Freshwater Biomedical Sciences Center, \\ Great Lakes WATER Institute, University of Wisconsin-Milwaukee \\ Milwaukee, WI
}

\begin{abstract}
TCDD is a reproductive toxicant and endocrine disruptor, yet the mechanisms by which it causes these reproductive alterations are not fully understood. In order to provide additional insight into the molecular mechanisms that underlie TCDD's reproductive toxicity, we assessed TCDDinduced transcriptional changes in the ovary as they relate to previously described impacts on serum estradiol concentrations and altered follicular development in zebrafish. In-silico computational approaches were used to correlate candidate regulatory motifs with observed changes in gene expression. Our data suggest that TCDD inhibits follicle maturation via attenuated gonadotropin responsiveness and/or depressed estradiol biosynthesis, and that interference of estrogen-regulated signal transduction may also contribute to TCDD's impacts on follicular development. TCDD may also alter ovarian function by disrupting various signaling pathways such as glucose and lipid metabolism, and regulation of transcription. Furthermore, events downstream from initial TCDD molecular-targets likely contribute to ovarian toxicity following chronic exposure to TCDD. Data presented here provide further insight into the mechanisms by which TCDD disrupts follicular development and reproduction in fish, and can be used to formulate new hypotheses regarding previously documented ovarian toxicity.
\end{abstract}

Keywords: TCDD, ovary, zebrafish, follicular development, microarray, endocrine disruptors

\title{
Introduction
}

2,3,7,8-Tetrachlorodibenzo-p-dioxin (TCDD) is a persistent environmental contaminant that is a known reproductive toxicant and

Reproductive Toxicology, Vol. 25, No. 1 (January 2008): pg. 47-57. DOI. This article is @ Elsevier and permission has been granted for this version to appear in e-Publications@Marquette. Elsevier does not grant permission for this article to be further copied/distributed or hosted elsewhere without the express permission from Elsevier. 
endocrine disruptor in nearly all vertebrates. The effects of TCDD on reproduction and fertility have been studied extensively $[\underline{1}-\underline{3}]$, and evidence suggests that TCDD compromises both ovarian function and follicular development. Female reproductive development is highly complex, and is synchronized by intricate and highly regulated signal transduction pathways that are integrated with the endocrine system. This complexity has made it particularly difficult to identify the molecular action of TCDD-induced ovarian toxicity. Although it is clear that TCDD impacts maturation and ovulation of ovarian follicles, as well as estradiol secretion [4-]], the mechanisms that underlie these reproductive toxicities are complicated and poorly understood.

It is generally accepted that TCDD toxicity is mediated by the aryl hydrocarbon receptor (AHR)-signaling cascade [ $\underline{8}]$. The ligandbound AHR complex is translocated to the nucleus where it dimerizes with the aromatic hydrocarbon receptor nuclear translocator (ARNT) protein. This heteromeric complex binds to the aryl hydrocarbonresponse element (AHRE) (TnGCGTG DNA motifs) located in the regulatory regions of several genes (e.g., , cyp1a1) and can initiate or suppress gene transcription [9-11]. While it is thought that such transcriptional regulation mediates the toxicity of TCDD, specific roles of these gene changes in dioxin-induced toxicity are not understood. Additionally, some actions of TCDD may be AHR-independent and/or result from downstream transcriptional changes.

Evidence suggests that disruptions in female reproduction by TCDD are likely the result of a direct effect at the ovary [12-17]. Since the AHR is expressed in the ovary, TCDD could disrupt critical cellular signals that regulate follicular development and/or estradiol biosynthesis via AHR-mediated alterations in gene transcription, thereby contributing to the observed decrease in ovarian development and reduced reproductive capacity. Alternatively, TCDD could interfere with the hypothalamic-pituitary-gonadal (HPG) axis or estradiol metabolism. For example, TCDD could negatively regulate estrogen signaling by inducing oxidative metabolism of estrogens via the AHRpathway, by suppressing the expression and/or efficacy of the estrogen receptors, or by inhibiting estradiol-regulated gene

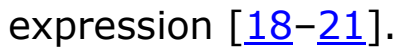

Reproductive Toxicology, Vol. 25, No. 1 (January 2008): pg. 47-57. DOI. This article is (c) Elsevier and permission has been granted for this version to appear in e-Publications@Marquette. Elsevier does not grant permission for this article to be further copied/distributed or hosted elsewhere without the express permission from Elsevier. 
Since the basic features of the HPG axis and AHR-signaling pathways in fish are fundamentally similar to other vertebrates [2224], fish are excellent model systems with which to investigate the effects of endocrine-disrupting chemicals on vertebrate reproductive function. The zebrafish has proven to be an effective system for investigation into the teratogenic effects of TCDD [느-27]. Zebrafish are highly prolific with rapid follicular development, and many of the receptors, enzymes, and peptide growth factors involved in follicular development have been characterized (see Ge 2005 for review); therefore, it is particularly suited for investigating TCDD's effects on the regulation of follicular development.

We have previously demonstrated that sublethal dietary exposure to TCDD alters follicular development, egg production, and serum $17 \beta$ estradiol concentrations in zebrafish [4]. Here we investigate the transcriptional events in the ovary that precede these previously described histomorphologic and physiologic alterations. We used quantitative RT-PCR to assess the effects on the expression of several candidate genes important in the regulation of follicle development, oocyte maturation, and vitellogenesis, and used cDNA microarray technology to evaluate altered gene expression profiles to identify other cellular pathways potentially impacted by TCDDexposure. Additionally, we used a functional genomics approach to examine candidate regulatory motifs in relation to different expression profiles in an effort to better clarify potential mechanisms of toxicity following chronic exposure to TCDD.

\section{Materials and Methods}

\section{Experimental animals}

Adult female ( $A B$ strain, Zebrafish International Resource Center) and male zebrafish (golden longfin, Ekwill Farms) were housed separately and acclimated for several weeks prior to the initiation of experiments. Fish were maintained at $26-28^{\circ} \mathrm{C}$ on a 14 -hour light and 10-hour dark cycle in a flow-through buffered, de-chlorinated water system and were spawned once weekly during the experiment.

Reproductive Toxicology, Vol. 25, No. 1 (January 2008): pg. 47-57. DOI. This article is (c) Elsevier and permission has been granted for this version to appear in e-Publications@Marquette. Elsevier does not grant permission for this article to be further copied/distributed or hosted elsewhere without the express permission from Elsevier. 
NOT THE PUBLISHED VERSION; this is the author's final, peer-reviewed manuscript. The published version may be accessed by following the link in the citation at the bottom of the page.

\section{TCDD exposure and RNA extraction}

Trout chow yielding a final concentration of approximately 0,10 , 40, or $100 \mathrm{ng} \mathrm{TCDD} / \mathrm{g}$ food ( $\mathrm{ppb}$ ) was prepared as previously described [28]. Females were fed brine shrimp nauplii daily, and contaminated trout chow diet 5 of 7 days per week. Fish were fed en masse to satiation, and based upon the average food consumed per fish, received an estimated dose of $0,0.08,0.32$, or $0.80 \mathrm{ng}$ TCDD/female/day. Based upon our previous work, fish accumulated approximately $0,0.6,3$, and $14 \mathrm{ng} \mathrm{TCDD} / \mathrm{g}$ fish after 15 days, and ovaries accumulated approximately $0.4,2$, and $5 \mathrm{ng} \mathrm{TCDD} / \mathrm{g}$ ovary (body burdens were not measured in this study). Following dietary exposure, five females from each treatment group were euthanized and ovaries were extracted and frozen in liquid nitrogen. Total RNA was isolated from individual ovaries using Trizol reagent (Invitrogen) and purified using an RNeasy MinElute cleanup kit (Qiagen) according to manufacturer's instructions. Individual samples were analyzed by agarose gel electrophoresis to confirm the integrity of the $18 \mathrm{~S}$ and $28 \mathrm{~S}$ ribosomal RNAs, and quantified by UV spectrophotometry at $260 \mathrm{~nm}$ using a Nanodrop ND-1000 Spectrophotometer (Nanodrop Technologies).

\section{Quantitative RT-PCR (QPCR)}

QPCR was used to quantify selected gene transcripts important in both receptor- and non-receptor-mediated regulation of follicular development, as well as estradiol biosynthesis, included: luteinizing hormone receptor (Ihr), follicle-stimulating hormone receptor (fshr), estrogen receptors (esr1, esr2a, esr $2 b$ ), epidermal growth factor (egf), egf receptor (egfr), inhibins (inhbba, inhbb), steroidogenic acute regulatory protein (star), side chain cleavage enzyme (cyp11a1), and aromatase (cyp19a1a). cDNA was synthesized from $5 \mu \mathrm{g}$ of total RNA from individual samples ( $n=3$ from each treatment group) using SuperscriptII reverse transcriptase (Invitrogen) and random hexamer primers following manufacturer's instructions. QPCR was performed using the Stratagene M×3000 system and the Full Velocity SYBR Green QPCR Master Mix with the manufacturer's instructions (Stratagene) using gene-specific primers listed in Table 1. Agarose gel electrophoresis and melting curve analysis confirmed that specific

Reproductive Toxicology, Vol. 25, No. 1 (January 2008): pg. 47-57. DOI. This article is @ Elsevier and permission has been granted for this version to appear in e-Publications@Marquette. Elsevier does not grant permission for this article to be further copied/distributed or hosted elsewhere without the express permission from Elsevier. 
products of expected size were amplified. All QPCR reactions were run in triplicate with negative (no template) controls. Relative gene expression data (fold-change) was calculated using the comparative quantification model [29] assuming 100\% efficiency, using small nuclear ribonuclear protein D1 polypeptide (snrpd1) as the reference gene; snrpd1 is important for mRNA processing, and expression was unaltered by TCDD exposure. QPCR on individual samples ( $n=3$ from each treatment group) was also used to verify microarray expression profiles. The gene-specific primers for these transcripts (cluster 4: cyp1a1; cluster 2: sepp1a, Igals3l, and krt4; cluster 1: krml and vtg1; cluster 3: tfa) are also listed also in Table 1. The seven transcripts showed altered expression by at least 2-fold following exposure to at least one dose of TCDD, and were selected for microarray verification based upon their functional characterization, presence of candidate regulatory motifs, and to represent each of the clusters in gene expression (see details below and in Table 2).

Table 1. Primer sequences used for QPCR. Gene names are listed in the text and Supplementary Tables.

\begin{tabular}{|c|c|c|}
\hline \multicolumn{2}{|c|}{ Gene symbol Sense Primer } & \multirow{2}{*}{$\begin{array}{l}\text { Antisense Primer } \\
\text { GTCTTCGCATGTGTTGATAAGAG }\end{array}$} \\
\hline cyp1a1 & ACTGGTGCGACTGGTTAATATGAG & \\
\hline cyp11a1 & CAGGTITTCACTGGAATCGG & CTGGTTAAAGATGCCATCCC \\
\hline cyp19a1a & ACTTCCAGAAAAATGTTCCGAGTC & TCTGAGGATAAGCTGCACGC \\
\hline egf & GCATCCAGAGAATGAACCTCGACGG & TGATTTGTCCTGTTTGTGTGTCCGC \\
\hline egfr & СTTACAAAGCCTGGAACGAAGAGCC & CCTGACACACTTTCTITCCGGCG \\
\hline esr1 & TCCACATCCACACAGTAGGC & СТTTCTTGATCAGGGTGGGG \\
\hline esr2a & AAGCTGCTGTGTCTGCTGGACTCGG & TCATGCAGTGCAGGTGGTCCATGCC \\
\hline$e s r 2 b$ & GGTGAAGTGTGTCATTTCTGTGCCG & GTGTGATTTTTGGGGCTGGGC \\
\hline fshr & AGCCGATTTGGATGCTTTAAAAGGC & TCAGACAGATGTCAGTGCACTTGGG \\
\hline inhbaa & GCACATTCAGAAGCCGACTGCC & TAAAGTCCGTCTGCCTGTGCGC \\
\hline$i n h b b$ & CGAATTCGGTGGACAGACAAACGG & TCCGTTCATTATTAGGCTCGCGG \\
\hline$k r m / 2.2$ & TATAAACTCAAGTGCGAAAGGC & ACCAGCAAACAATCTTATGC \\
\hline krt4 & CAAAACCAGTGTCACCACCG & САТСТССТСАTTAACAGGGG \\
\hline lgals3l & GCTGTACAAGTGCATGTAAAGGG & CGTCTIITATGCATGAAGCG \\
\hline Ihr & CAAAAAGGACGAGTCGCTGAAACGC & GCAGAAGAAAAACAAGAAGCAGGGC \\
\hline seppa1 & AAATCTGACTITAACTGGTCCAGTG & ATGTTACATGACCTTTGCCC \\
\hline snrpd1 & CGTCACGATTGAGTGAAGAATGGC & TGAGATCTTCATCTCCTCGGCCC \\
\hline star & ACCCACCTGTATTGTCATGCG & AATGGCTGCGTCTATACCCC \\
\hline tfa & ATTAAGCACACTGTGGTCGG & AGCATGAACTGGCACTTGGG \\
\hline$v \operatorname{tg} 1$ & GAGATTGAACTGACTGCAGCC & ATTCCACATGAACATAGGCC \\
\hline
\end{tabular}

Reproductive Toxicology, Vol. 25, No. 1 (January 2008): pg. 47-57. DOI. This article is @ Elsevier and permission has been granted for this version to appear in e-Publications@Marquette. Elsevier does not grant permission for this article to be further copied/distributed or hosted elsewhere without the express permission from Elsevier. 
NOT THE PUBLISHED VERSION; this is the author's final, peer-reviewed manuscript. The published version may be accessed by following the link in the citation at the bottom of the page.

Table 2. List of representative transcripts identified by microarray to be dysregulated greater than 2-fold in one or more treatment group. Presence of putative AHRE or ERE is noted. See Supplementary Tables 1- $\underline{3}$ for complete gene list plus location of putative response elements.

\section{Gene symbol 10 ppb 40 ppb 100 ppb AHRE ERE}

Ovarian development

$\begin{array}{lllclll}\text { fabp3 } & -2.92 & -2.68 & -4.14 & & \\ \text { tpte } & -1.91 & -1.45 & -2.60 & * & \\ \text { tph1l } & -2.64 & -1.53 & 1.19 & * & \\ \text { vtg1 } & -\mathbf{7 . 3 8} & \mathbf{- 1 . 6 2} & \mathbf{- 1 . 8 3} & * & *\end{array}$

Detoxification/oxidative stress

$\begin{array}{lccccc}\text { cyp1a } & \mathbf{6 . 9 3} & \mathbf{1 4 . 8 7} & \mathbf{2 4 . 6 8} & * & * \\ \text { sepp1a } & -1.71 & -1.21 & -2.48 & & \\ \text { serpina1 } & 29.44 & 1.51 & 3.07 & * & *\end{array}$

Lipid metabolism

$\begin{array}{lccccc}\text { apoeb } & -1.83 & -1.09 & -4.12 & * & \\ \text { apom } & 6.16 & 1.04 & 1.32 & & \\ \text { lipf } & -2.06 & -1.85 & -2.30 & * & *\end{array}$

Carbohydrate metabolism

$\begin{array}{lllll}\text { Idha } & -1.78 & -1.45 & -2.59 & *\end{array}$

Immune response/cellular repair

$\begin{array}{lccccc}\text { anxa1a } & -2.20 & -3.36 & -7.06 & * & * \\ c p & 24.49 & 1.06 & 2.16 & * & \\ \text { fgb } & 12.80 & -1.82 & -1.35 & * & \\ \text { fgb } & 5.66 & 1.43 & 1.61 & * & * \\ \text { tfpib } & -1.54 & -1.95 & -5.66 & & \\ \text { timeless } & -2.92 & 1.88 & 1.07 & * & \\ \text { Structure } & & & & & \\ \text { krt4 } & \mathbf{- 1 . 9 6} & \mathbf{- 2 . 9 3} & -\mathbf{5 . 6 5} & * & * \\ \text { Igals3I } & \mathbf{- 1 . 8 9} & \mathbf{- 2 . 6 8} & \mathbf{- 2 . 5 6} & * & \end{array}$

Signal transduction/transcription/regulation of cell cycle

$\begin{array}{lccccc}\text { rab5b } & 2.45 & 1.34 & 2.13 & * & * \\ \text { rad1 } & -1.10 & 1.13 & -2.16 & & \\ \text { rbp2a } & -2.98 & -2.39 & -4.54 & * & * \\ \text { Igfbp1 } & 5.58 & -1.38 & -1.47 & * & \\ \text { Junb } & -2.21 & -1.88 & -4.39 & * & * \\ \text { krmI2.2 } & -\mathbf{2 . 5 9} & -\mathbf{1 . 1 9} & -\mathbf{2 . 4 1} & * & * \\ \text { Iztfl1 } & -1.12 & -1.12 & -2.33 & * & \\ \text { sp4 } & 1.51 & 2.32 & 1.96 & * & \\ \text { ing3 } & -2.29 & 1.17 & -1.32 & & \\ \text { igfbp1 } & 5.58 & -1.38 & -1.47 & * & \\ \text { tfa } & \mathbf{1 1 . 6 4} & \mathbf{- 2 . 0 9} & \mathbf{- 1 . 3 2} & * & \end{array}$

Reproductive Toxicology, Vol. 25, No. 1 (January 2008): pg. 47-57. DOI. This article is (C) Elsevier and permission has been granted for this version to appear in e-Publications@Marquette. Elsevier does not grant permission for this article to be further copied/distributed or hosted elsewhere without the express permission from Elsevier. 
NOT THE PUBLISHED VERSION; this is the author's final, peer-reviewed manuscript. The published version may be accessed by following the link in the citation at the bottom of the page.

\section{Gene symbol 10 ppb 40 ppb 100 ppb AHRE ERE}

ptena

$-2.32$

1.51

1.38

Gene symbols are from Affymetrix probe identifiers and are organized into general functional groups. Transcripts in bold were verified by QPCR.

\section{Microarray hybridization and analysis}

Differential ovarian gene expression was determined using the Affymetrix Zebrafish Genome Array, which represents approximately 14,900 transcripts. Experiments were designed to comply with MIAME guidelines [30]. In an effort to reduce variability, target synthesis using pooled RNA (of equal quantity and comparable quality from 5 females per treatment group) [31-33], biotin-labeling, hybridizations, and staining were performed using standard Affymetrix reagents and methods (http://www.affymetrix.com/index.affx). Pooled samples were run in duplicate such that a total of eight arrays were used.

Images were extracted from TIFF files using Microarray Suite Version 5.0 (Affymetrix). Raw data can be viewed at the National Center for Biotechnology Information Gene Expression Omnibus website (http://www.ncbi.nlm.nih.gov/projects/geo/); series record GSE4859. Data were transformed and normalized using GeneSpring software (Agilent Technologies) and further analyzed using Bioconductor 1.6 [34]. Expressed data were obtained by selecting 8732 probes present in all eight chips. Differentially expressed probes were identified as those showing at least a 2-fold change in expression in both replicates of one or more of the treatment groups compared with control. The dysregulated transcripts were assigned to general functional groups based upon their Gene Ontology terms, or an annotated putative $H$. sapiens or $M$. musculus ortholog from SwissProt (http://us.expasy.org/sprot/). The dysregulated transcripts were clustered using agglomerative hierarchical clustering [35] without performing mean centering of expression values, and using Ward's method for merging clusters.

\section{In-silico Transcription Factor Analysis}

Possible cis-acting AHRE (GCGTG) and ERE (AGGTCAnnnTGACCT) sequences were identified within the regulatory region of differentially expressed genes as well as candidate genes

Reproductive Toxicology, Vol. 25, No. 1 (January 2008): pg. 47-57. DOI. This article is @ Elsevier and permission has been granted for this version to appear in e-Publications@Marquette. Elsevier does not grant permission for this article to be further copied/distributed or hosted elsewhere without the express permission from Elsevier. 
determined by QPCR. The reference genes were 22877 RefSeq genes and Ensemble predictions mapped to the draft zebrafish genome, and the test sequences were RefSeqs overlapping with consensus sequences of differentially expressed Affymetrix probes. Of the dysregulated transcripts, 140 could be mapped to Ensemble genes and were used for subsequent analyses. The $5^{\prime}$ regulatory sequences of the reference and dysregulated genes were modeled by the $5^{\prime}$-UTR of each RefSeq (5000 bp upstream sequences from the transcriptional start site). Regulatory sequences were acquired from the June 2004 draft assembly of the zebrafish genome using the University of California - Santa Cruz table browser [36] and were scanned for possible transcription factor binding sites using TFBS [37], JASPAR [38] for AHRE sequences at a 100\% scoring threshold, and TRANSFAC Public 7.0 [39] for ERE sequences at an $80 \%$ scoring threshold. To compare control and differentially expressed sequences, the hypergeometric distribution in R 2.1.0 was used to describe the probability of drawing a sample the same size as the differentially expressed sequences from the control sample with the same or fewer transcription factor binding sites identified. A chi-squared test was used to determine if there was a location bias of transcription factor binding sites with respect to identified clusters. The starting position distributions of AHREs and EREs within a cluster were compared with the corresponding distributions in the entire Ensemble known gene collection. Distributions for a cluster significantly different $(p<0.05)$ from control suggested putative regulatory patterns.

\section{Data analysis}

Statistical analysis of QPCR data was performed using SigmaStat 2.0 (SPSS, Inc) and presented as means \pm standard error of the mean (SEM). Data were evaluated for homoscedasticity (Levene Median test) and one-way analysis of variance (ANOVA) was used to detect treatment-related effects on the expression of each candidate transcript. Where significant differences were indicated between treatment groups, and the data were homogeneous, pair-wise multiple comparisons were conducted using the Tukey test. When tests for homogeneous variance failed, the Kruskal-Wallis one way ANOVA on ranks was used, and significant differences were evaluated using the Dunn's test. Pearson's correlation was used to determine whether

Reproductive Toxicology, Vol. 25, No. 1 (January 2008): pg. 47-57. DOI. This article is @ Elsevier and permission has been granted for this version to appear in e-Publications@Marquette. Elsevier does not grant permission for this article to be further copied/distributed or hosted elsewhere without the express permission from Elsevier. 
changes in gene expression identified by microarray were correlated with changes in expression of the seven transcripts determined by QPCR. For all analyses, significant differences were identified at $p<$ 0.05 .

\section{Results}

\section{Effects on the regulation of follicular development and estradiol biosynthesis}

Dietary TCDD exposure suppressed the expression of all examined candidate transcripts that are important in both receptorand non-receptor-mediated regulation of follicular development, as well as estradiol biosynthesis (Figure 1A). The candidate genes were down-regulated by at least 2-fold; overall changes in expression were not dose-dependent. Locations of putative AHREs and EREs within the regulatory region of the transcripts are illustrated in Figure $1 \mathrm{~B}$.

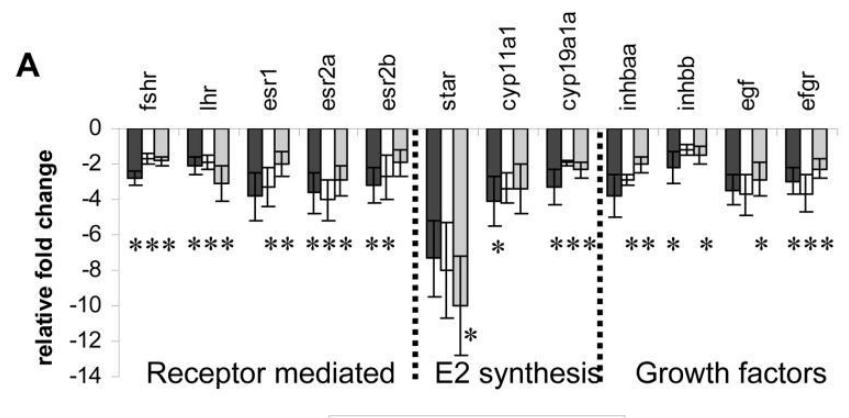

๑ 10ppb $\square 40 \mathrm{ppb} \square$ 100ppb

B

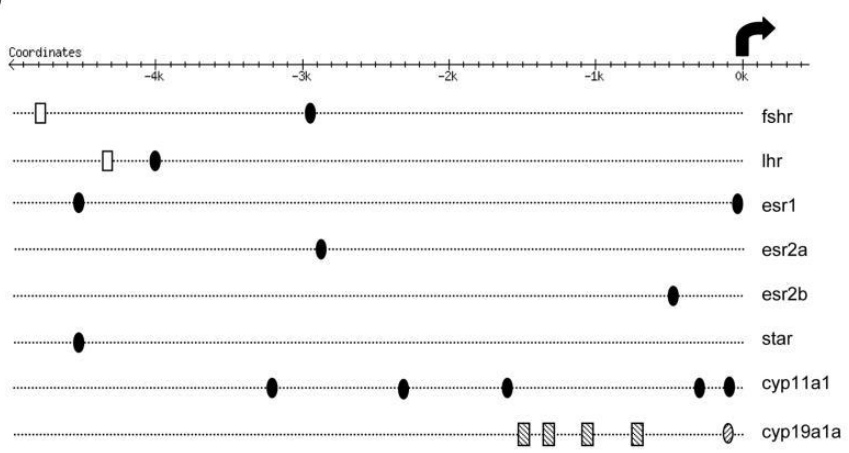

Figure 1. A. Fold changes in expression of several genes important for follicular development determined by QPCR; normalized to snrpd1. *Denotes significant changes in expression compared with control $(p<0.05)$; dose-dependent changes

Reproductive Toxicology, Vol. 25, No. 1 (January 2008): pg. 47-57. DOI. This article is @ Elsevier and permission has been granted for this version to appear in e-Publications@Marquette. Elsevier does not grant permission for this article to be further copied/distributed or hosted elsewhere without the express permission from Elsevier. 
NOT THE PUBLISHED VERSION; this is the author's final, peer-reviewed manuscript. The published version may be accessed by following the link in the citation at the bottom of the page.

were not observed. B. Location of putative AHREs (filled ovals, this study; lined ovals [86]; putative EREs (empty rectangles), and putative 1/2-EREs (lined rectangles [86] within upstream regulatory regions. Arrow represents the transcriptional start site.

\section{Effects of TCDD on Global Ovarian Gene Expression}

Two hundred thirty-five probe sets (representing 229 unique transcripts) were identified as being either induced or suppressed greater than 2-fold following exposure to one or more TCDD concentration. Overall, more transcripts were suppressed than were induced with $37 \%(85 / 229), 7 \%(16 / 229)$, and $40 \%(92 / 229)$ of the transcripts down-regulated and $11 \%$ (25/229), 4\% (9/229), and $11 \%$ $(25 / 229)$ of the transcripts up-regulated in the 10,40 , and $100 \mathrm{ppb}$ treatment groups, respectively. QPCR using individual samples verified microarray results for seven transcripts (Figure 2). The direction and fold-change values for samples from individual animals were correlated with changes in expression determined by microarray analysis of pooled samples for all seven genes $(p<0.01)$, demonstrating that our approach successfully identified changes in gene expression across treatment groups that could be validated with individual samples.

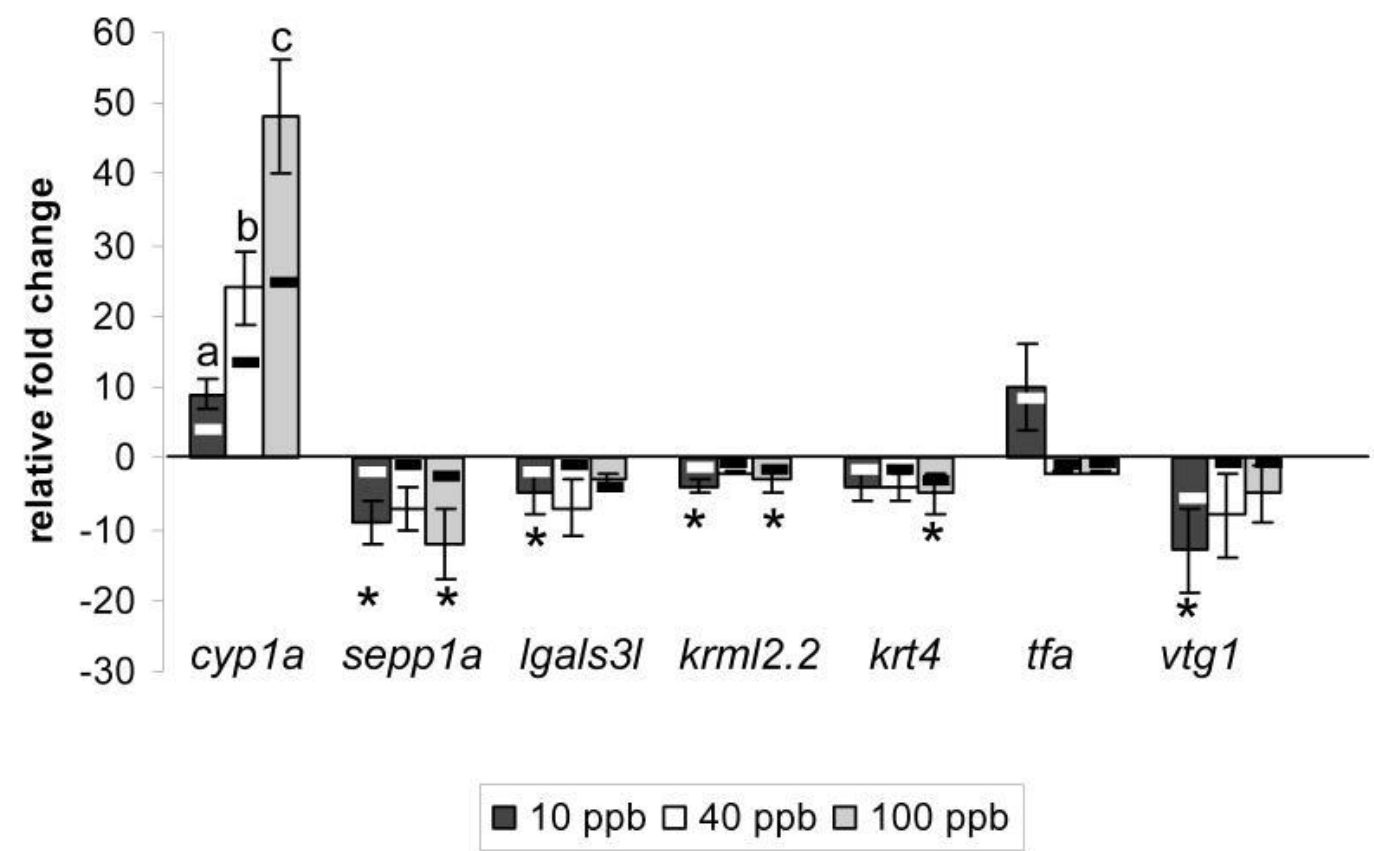

Figure 2. Validation of microarray results. Bars represent mean relative fold changes in gene expression determined by QPCR; normalized to snrpd1. Letters or * denote significant differences for that gene compared with control. Black and white lines

Reproductive Toxicology, Vol. 25, No. 1 (January 2008): pg. 47-57. DOI. This article is @ Elsevier and permission has been granted for this version to appear in e-Publications@Marquette. Elsevier does not grant permission for this article to be further copied/distributed or hosted elsewhere without the express permission from Elsevier. 
represent fold-changes in expression determined by array analysis. (Pearson's correlation, $\mathrm{p}<0.01$ ).

Differentially expressed transcripts with known functions (103/229) were assigned to general functional groups based upon the top three Gene Ontology (GO) functions and known biologic functions, and are important for signal transduction/transcription/regulation of the cell cycle, as well as for glucose and lipid metabolism, immune response/cellular repair, and structure (Table 2 and Figure 3 ). Hierarchical clustering grouped transcripts into one of five clusters (Figure 4A and Supplementary Table 1). On average, the 17 transcripts within the fifth cluster show minimal changes in gene expression across treatment groups and are therefore not shown in Figure 4 or discussed further (due to lack of statistical power). The majority of functionally annotated transcripts within clusters one and four regulate gene transcription or are important for signal transduction, while most functionally annotated transcripts within cluster two are important for maintaining structure, and those within cluster three play a role in immune function (see Figure 4A).

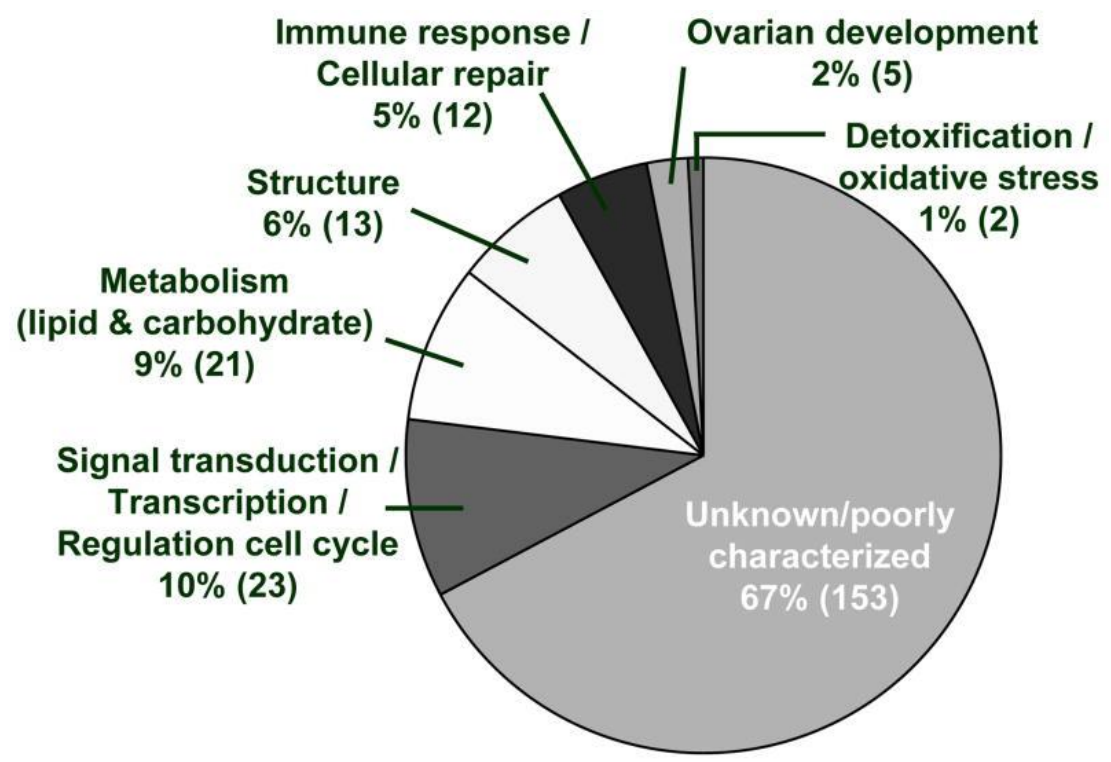

Figure 3. Proportion of dysregulated ovarian transcripts within each general functional group. The number in parentheses represents the number of transcripts out of 229 within each functional group.

Reproductive Toxicology, Vol. 25, No. 1 (January 2008): pg. 47-57. DOI. This article is (c) Elsevier and permission has been granted for this version to appear in e-Publications@Marquette. Elsevier does not grant permission for this article to be further copied/distributed or hosted elsewhere without the express permission from Elsevier. 
A

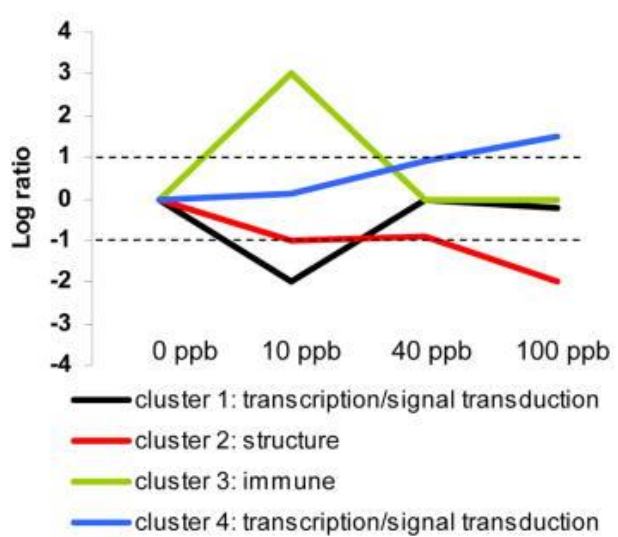

B

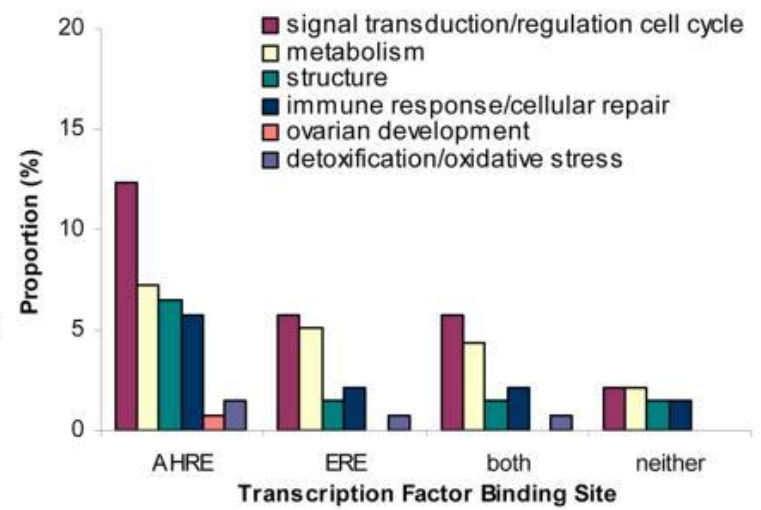

Figure 4. A. Gene expression (expressed as the log ratio compared with control) patterns of four hierarchical gene clusters including the primary functional group represented within each cluster. B. Proportion of dysregulated ovarian transcripts within each general functional group that contain putative AHREs, ERES, both or neither.

One hundred forty of the dysregulated transcripts were mapped to Ensemble genes, and $89 \%$ of these (125/140 corresponding to 113 unique transcripts) were found to have putative AHREs in the regulatory region of the gene (Figure 4B, Supplementary Tables 1 and 2), compared with $92 \%$ of the reference genes (21046/22877). Using hypergeometric distribution to calculate the probability of finding only 125 or fewer genes out of 140 that were drawn from 22877, our data suggest differentially expressed transcripts are not enriched for putative AHREs in $(p=0.14)$. Estimated probability density curves shown in Figure $5 \mathrm{~A}$ illustrate the potential location bias of putative AHREs for each cluster along with the location of putative AHREs for all genes in the zebrafish genome for comparison. Upstream regions of genes within cluster 2 have putative AHRE distributions that are significantly different $\left(p=2.9 \times 10^{-12}\right)$ from those of the control gene set, with AHREs distributed $3000-4000 \mathrm{bp}$ and $1500 \mathrm{bp}$ upstream of the transcriptional start site (TSS). In cluster 3, AHREs tend to be further upstream ( $3500 \mathrm{bp})$ from the TSS than control $(p=2.0 \times$ $10^{-7}$ ), and in cluster 4, AHREs tend to be concentrated within $2000 \mathrm{bp}$ upstream of the TSS $(p=0.01)$. Of the 113 unique transcripts that contain putative AHREs, $16 \%$ were upregulated and $51 \%$ were downregulated across treatment groups; many function as transcription factors and regulate the cell cycle (Figure 5).

Reproductive Toxicology, Vol. 25, No. 1 (January 2008): pg. 47-57. DOI. This article is (C) Elsevier and permission has been granted for this version to appear in e-Publications@Marquette. Elsevier does not grant permission for this article to be further copied/distributed or hosted elsewhere without the express permission from Elsevier. 
NOT THE PUBLISHED VERSION; this is the author's final, peer-reviewed manuscript. The published version may be accessed by following the link in the citation at the bottom of the page.

A

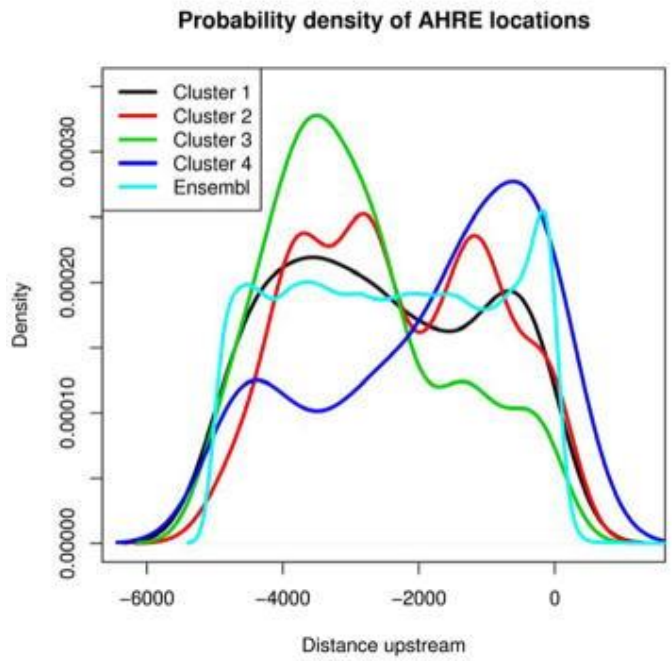

B

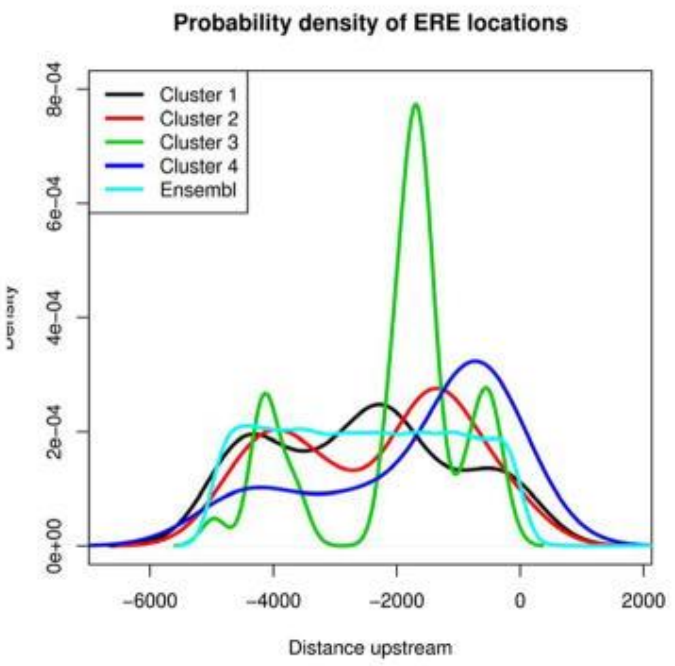

Figure 5. Probability density curves illustrate the potential location bias of putative AHREs (A) and EREs (B) with respect to the translational start site for the transcripts within each hierarchical cluster compared with reference. Note: Probability density curves represent the probability distribution in terms of integrals, illustrated as a line depicting the relative frequency that each response element is represented at that location.

Approximately $49 \%$ of the mapped dysregulated probes (68/140 corresponding to 58 unique transcripts) were found to have putative EREs in the regulatory region of the gene (Figure 4B, Supplementary Tables 1 and 3 ); $17 \%$ of these transcripts were upregulated and $55 \%$ were downregulated across treatment groups. Forty-two percent of the reference genes (9608/22877) contained putative EREs in the regulatory region; differentially expressed genes contained putative EREs at a much higher rate than would be expected $(p=0.05)$. Estimated probability density curves shown in Figure $5 \mathrm{~B}$ illustrate the potential location bias of putative EREs for each cluster. In cluster 1 , EREs tend to be centered around 2000 bp from the TSS ( $p=3.0 \times$ $\left.10^{-7}\right)$. In cluster 3 , EREs are largely concentrated at about 1800-2000 bp upstream of the TSS $\left(p=8.2 \times 10^{-7}\right)$ and in cluster 4, EREs tend to be concentrated within 2000 bp upstream $(p=0.02)$. Many of these transcripts function in carbohydrate and lipid metabolism, or regulation of transcription or the cell cycle (Figure 4B).

Approximately $41 \%$ of the dysregulated probes contain both putative AHRE and ERE in the regulatory region (Figure 4B, Supplementary Table 1); approximately $20 \%$ of these transcripts were upregulated, while the majority of the transcripts (51\%) were

Reproductive Toxicology, Vol. 25, No. 1 (January 2008): pg. 47-57. DOI. This article is @ Elsevier and permission has been granted for this version to appear in e-Publications@Marquette. Elsevier does not grant permission for this article to be further copied/distributed or hosted elsewhere without the express permission from Elsevier. 
downregulated across treatment groups. Approximately $40 \%$ of the reference genes (9039/22877) contain putative AHREs and EREs in the regulatory region; the dysregulated transcripts were not significantly enriched for both AHREs and EREs $(p=0.35)$. As with those with putative EREs, many of these transcripts are important for carbohydrate and lipid metabolism or function as transcription factors and regulate the cell cycle (Figure 4B).

\section{Discussion}

TCDD has been shown to perturb the regulation of vertebrate follicular development and ovulation, as well as steroidogenesis in the ovary $[\underline{3}-\underline{6} ; \underline{40}]$. Regulation of these processes is complex, integrating receptor-mediated hormonal signals from the pituitary with locally produced factors to form an intimate regulatory network within and between follicles. This poses a challenge for identifying the molecular mechanisms that regulate TCDD's reproductive toxicity. Furthermore, the sometimes subtle secondary and tertiary effects on gene expression following chronic dioxin exposure can be difficult to ascertain and to correlate with observed toxicities. This study represents one of the first attempts to characterize the effects on ovarian gene expression following exposure of fish to chronic, sublethal concentrations of TCDD. Using a candidate-gene approach in conjunction with a genomics approach, we have identified several novel dioxin-responsive genes that with further study may better clarify TCDD's ovarian toxicity.

\section{Effects on the regulation of follicular development and estradiol biosynthesis}

All of the candidate genes selected for QPCR analysis (those important for receptor-and non-receptor mediated regulation of follicular development and estradiol biosynthesis) show peak expression in follicles that are in the mid-to-late stages of vitellogenesis, and should therefore be highly expressed in whole ovary of cycling females under normal conditions [41-44]. Gonadotropins are important regulators of follicular development, and in zebrafish, have been shown to induce the expression of several other genes important for oocyte development $[\underline{41 ;} \underline{45}]$. Suppression of

Reproductive Toxicology, Vol. 25, No. 1 (January 2008): pg. 47-57. DOI. This article is @ Elsevier and permission has been granted for this version to appear in e-Publications@Marquette. Elsevier does not grant permission for this article to be further copied/distributed or hosted elsewhere without the express permission from Elsevier. 
both gonadotropin receptors suggests that impaired follicular development may be the result of this suppression. Similar effects on ovarian gonadotropin receptor expression have been shown in mammals $[\underline{6} ; \underline{46}-\underline{48}]$ and others suggest that TCDD's anovulatory effects are likely mediated directly at the ovary in an AHR-dependent

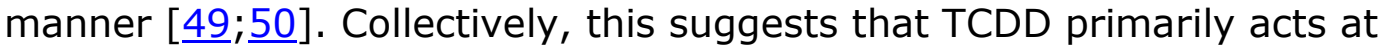
the ovary to suppress expression of gonadotropin receptors.

Exposure to TCDD also downregulates the expression of several genes important in estradiol biosynthesis (star, cyp11a1, and cyp19a1a), and likely contributes to the previously observed reduced serum estradiol concentrations [4]. While it has been proposed that reduced estradiol concentrations following exposure to TCDD may result from suppression of aromatase activity, the mechanism by which TCDD disrupts ovarian steroidogenesis is not clear. Baba et al [51] show that exposure to DMBA, an AHR agonist, is correlated with increased estradiol concentrations and increased aromatase expression, while others suggest that the target of TCDD-induced suppression of estradiol is upstream of aromatase in the steroidogenic pathway [52]. However, other in vitro and in vivo studies demonstrate that exposure to TCDD decreases the expression and activity of aromatase, and is correlated with decreased estradiol concentrations [53-55]. While our data support findings in mammalian systems that suggest TCDD-induced reductions in serum estradiol may result from diminished aromatase expression/activity, impairing estradiol biosynthesis, we cannot rule out star and cyp11a1 as potential targets. Others have demonstrated that expression of star and cyp11a1 is suppressed in the interregnal gland following exposure to $\beta N F$, lending support for these transcripts as other potential targets for endocrine disruption by AHR-ligands [트].

While not as well characterized in fish, estradiol also plays a role in regulating follicle development and ovulation via ERs in the ovary. Three forms of the ER (esr1, esr2a, esr $2 b$ ) are expressed in the zebrafish ovary, and all are capable of initiating transcription of genes $[\underline{57} ; \underline{58}]$. Here we show that all three forms of the ER are downregulated following chronic exposure to TCDD, as has been demonstrated for mammals $[\underline{59} ; \underline{60}]$. By suppressing the expression of ERs in the ovary, follicles may be unable to respond to estrogen signaling and/or induce the transcription of estrogen-responsive genes

Reproductive Toxicology, Vol. 25, No. 1 (January 2008): pg. 47-57. DOI. This article is (c) Elsevier and permission has been granted for this version to appear in e-Publications@Marquette. Elsevier does not grant permission for this article to be further copied/distributed or hosted elsewhere without the express permission from Elsevier. 
important for ovarian development [61-64]. Our array data further support this hypothesis in that more than half of transcripts with putative EREs were downregulated in the ovaries of TCDD-treated fish.

Finally, while follicular development is primarily controlled by gonadotropin hormones, various local factors released from granulosa and theca cells as well as from oocytes, also mediate gonadotropin signaling. Peptide growth factors such as inhibins and follistatins potentiate the action of gonadotropins and maturation-inducing steroid in the induction of final oocyte maturation [44]. Activin $\beta A$ (Inhbaa) promotes ovary and follicle growth, whereas activin $\beta B$ (Inhbb) exerts a tonic role throughout follicle development, and becomes critical at the late stage of oocytes maturation and/or ovulation [65]. Epidermal growth factor enhances the rate of oocyte maturation via gonadotropin signaling, inhibits apoptosis, stimulates follicle cell proliferation, and plays a role in controlling follicle survival and steroidogenesis $[66 ; 67]$. TCDD exposure suppressed the expression of both forms of inhibin, as well as egf and its receptor, although not significantly at all doses, suggesting that impacts further upstream (e.g., expression of gonadotropin receptors) may have a greater impact on follicular development.

\section{Effects of TCDD on Global Ovarian Gene Expression}

While our microarray experimental design does not allow us to make direct conclusions about the mechanisms that underlie TCDD's ovarian toxicity, we are still able to offer valuable insights into additional cellular pathways and signal cascades that are altered by chronic exposure to TCDD. TCDD disrupts several integrated cellular pathways (including structure, glucose and lipid metabolism, immune response, and regulation of transcription) reaffirming the complexity of TCDD toxicity and identifying several new avenues for further study. For example, chronic exposure to $10 \mathrm{ppb}$ TCDD shows a trend for greater alterations in the expression of genes important for transcription/signal transduction (cluster 1 ) and immune response genes (cluster 3) compared to chronic exposure to 40 and $100 \mathrm{ppb}$ TCDD. Exposure to $10 \mathrm{ppb}$ TCDD for 15 days resulted in reduced estradiol concentrations in zebrafish, but follicular development, overall egg production and spawn success were not altered; follicular

Reproductive Toxicology, Vol. 25, No. 1 (January 2008): pg. 47-57. DOI. This article is @ Elsevier and permission has been granted for this version to appear in e-Publications@Marquette. Elsevier does not grant permission for this article to be further copied/distributed or hosted elsewhere without the express permission from Elsevier. 
development, egg production and spawn success was reduced following exposure to 40 and 100 ppb TCDD for 15 days [4]. Therefore, the roles that such cellular pathways play in the modulation by TCDD of follicular development and reproductive success by TCDD warrants further study.

Following chronic exposure to TCDD, we show that transcripts such as keratins ( $k r t 4,8$, and 18), collagens (col1a2, col5a2l), lectins (Igals3/ and Igals1/2), and actins (acta2) necessary for maintaining structural integrity were suppressed. We verified the expression profiles for two of these transcripts ( $k r t 4$ and Igals3l); expression profiles for similar transcripts (cluster 2) suggest a trend for a dosedependent suppression of structure-related transcripts. TCDD has also been shown to impact the expression of genes important for maintaining and metabolizing the extracellular matrix in the regenerating fin of zebrafish [ 68] and in the liver of medaka [ 69]. Since TCDD exposure is associated with wasting-syndrome, decreased ovarian somatic index, and inhibited regenerative growth of the fin and liver, the impacts of TCDD on the expression of structural proteins warrants further consideration as an integral component of the sublethal toxic response to TCDD.

Many of the ovarian transcripts disrupted by TCDD are important for regulation of the cell cycle and signal transduction. Genes such as cyp1a1 (cluster 4) show a trend for a dose-dependent increase in their suppression, while others such as the large Maf protein $k r m / 2.2$ (cluster 1 ) are downregulated following chronic exposure to $10 \mathrm{ppb}$ TCDD but not 40 or $100 \mathrm{ppb}$ TCDD. Most of the genes within cluster $4(15 / 17)$ have putative AHREs, and cyp1a1 is known to be induced by exposure to TCDD in an AHRE-mediated manner; therefore, investigation into whether the expression of such transcripts is induced by TCDD in an AHRE-mediated manner warrants further study. Large Maf proteins such as $\mathrm{krm} / 2.2$ are important for regulation of cell differentiation. While the function of large Maf proteins in fish ovarian development is not known, $\mathrm{krm} / 2.2$ plays a role in the differentiation of cell lineages in developing embryos [궁, and large Maf proteins have been shown to be key regulators of gonad morphogenesis in Drosophila [71]. Expression of another gene, tfa (cluster 3 ) is also increased following chronic exposure to $10 \mathrm{ppb}$ TCDD. In mammals, transferrins have an inhibitory effect on FSH-

Reproductive Toxicology, Vol. 25, No. 1 (January 2008): pg. 47-57. DOI. This article is @ Elsevier and permission has been granted for this version to appear in e-Publications@Marquette. Elsevier does not grant permission for this article to be further copied/distributed or hosted elsewhere without the express permission from Elsevier. 
induced differentiation of granulosa cells $[\underline{72 ; 73}]$. Alterations in such transcripts may account for observed alterations in follicular development, and warrant further investigation. Similar impacts on the expression of transcripts important for signal transduction and regulation of cellular differentiation have been noted by others to occur in embryonic heart and regenerating tail of zebrafish $[\underline{68} ; \underline{74} ; \underline{75}]$ as well as liver, brain, and testis of medaka [76]. While the specific transcriptional profiles within these different tissue types following exposure to TCDD reflect tissue-specific targets of TCDD, overall impacts on the regulation of important signaling molecules and transcription factors likely constitute a common denominator in TCDD's toxicity.

Our work also identifies several novel pathways by which chronic exposure to TCDD may alter ovarian development. For example, both glucose and lipid metabolism may be altered in the ovary as a result of TCDD exposure. Since glucose and lipids play active roles in oogenesis and egg quality in fish [77], perhaps TCDDinduced disruption of these pathways contributes to the observed impacts on ovarian development [4] as well as offspring survival [28]; this hypothesis warrants further consideration. Several genes important for immune response also appear altered by TCDD exposure. Alterations in the expression of transcripts involved in immune function could induce an inflammatory response, negatively impacting steroidogenesis and follicular development [궁, or perhaps interfere with normal ovarian development by restricting the removal of apoptotic cells or cellular debris [79]. Such transcriptional changes could also reflect responses from immune cells as the result of tissue repair, as suggested by Volz et al. [69]. Additional studies are necessary to elucidate the impacts of altered immune response on ovarian development in fishes.

\section{Insights into the mechanisms of TCDD-induced ovarian toxicity}

In-silico analysis of the regulatory region of dysregulated transcripts have enabled us to further support hypotheses regarding the mechanisms by which chronic exposure to TCDD induces ovarian toxicity and to identify new avenues for continued research into

Reproductive Toxicology, Vol. 25, No. 1 (January 2008): pg. 47-57. DOI. This article is (c) Elsevier and permission has been granted for this version to appear in e-Publications@Marquette. Elsevier does not grant permission for this article to be further copied/distributed or hosted elsewhere without the express permission from Elsevier. 
TCDD's sublethal toxic response in the ovary. The AHR-pathway remains active in zebrafish ovary following chronic exposure to TCDD, evidenced by increased cyp1a1 expression compared with control. While AHR-mediated changes in gene expression likely contribute to TCDD-induced ovarian toxicity, dysregulated transcripts identified by microarray do not appear to be enriched for AHREs compared with reference genes. While we cannot be sure which of these AHREs are functional, others have reported similar findings in that not all TCDDinduced changes in gene expression could be correlated with the presence of AHREs either by promoter analysis or determination of AHR-dependence $[\underline{69} ; \underline{80} ; \underline{81}]$. Collectively, these studies suggest that TCDD-induced histopathologic alterations likely involve changes in the expression of genes downstream from initial AHRE-activated transcription in addition to direct AHRE-mediated changes in gene expression (e.g., by altering the expression of transcription factors and signaling proteins via the AHRE, leading to an alteration in the expression of their target genes in a tissue-specific manner).

Our data also suggest that impacts on the expression of estradiol-regulated genes may also be important for TCDD-induced ovarian toxicity. Several propose that interactions between AHR and ER pathways are inhibitory, and that AHR-ligands could suppress expression of genes via the AHRE $[\underline{19} ; \underline{82} ; \underline{83}]$. While dysregulated transcripts identified by microarray are enriched for putative EREs, presence of both AHRE and ERE was not different than for reference genes; therefore no conclusions regarding potential interaction/interference between the two signaling pathways can be drawn from these data. Others suggest that when estrogen is present, AHR ligands can attenuate estrogen signaling at an ERE site, and that while the AHR is involved, the mechanism of action is independent from AHRE-mediated signaling $[20 ; 84 ; \underline{85}]$. Our findings here lend support to this hypothesis. Putative EREs were identified in the promoter regions of $I h r, f s h r$, and cyp19a1a, of which only cyp19a1a has been demonstrated to be estrogen responsive in zebrafish [84]. Further, dysregulated transcripts identified by microarray appear to be enriched for EREs compared with reference genes. Collectively these studies suggest that the antiestrogenic actions of TCDD at the ovary entail multiple and perhaps gene/promoter-specific molecular mechanisms involving ER/AHR cross-talk, and warrant further study.

Reproductive Toxicology, Vol. 25, No. 1 (January 2008): pg. 47-57. DOI. This article is @ Elsevier and permission has been granted for this version to appear in e-Publications@Marquette. Elsevier does not grant permission for this article to be further copied/distributed or hosted elsewhere without the express permission from Elsevier. 
NOT THE PUBLISHED VERSION; this is the author's final, peer-reviewed manuscript. The published version may be accessed by following the link in the citation at the bottom of the page.

\section{Conclusions}

Environmental compounds that disrupt hormone signaling can exert a profound effect on reproduction. Our data suggest that TCDD inhibits expression of key genes that regulate follicular development and estradiol biosynthesis, and provide further evidence for a mechanistic link for impairment of reproduction in fish exposed to TCDD. Suppression of all of the candidate genes in this study following chronic exposure to TCDD is consistent with previously described reductions in serum $17 \beta$ estradiol concentrations and attenuated follicular development [4], and suggests that TCDD inhibits the transition of pre-vitellogenic follicles to vitellogenic follicles via attenuated gonadotropin responsiveness and/or reduced estradiol biosynthesis. Overall lack of a dose response suggests that the ovary is highly sensitive to TCDD, and that accumulations of less than $1 \mathrm{ng}$ $\mathrm{TCDD} / \mathrm{g}$ female are sufficient to reduce reproductive capacity of female zebrafish. Furthermore, TCDD appears to impact several integrated cellular pathways, illustrating the complex and profound effects it has on the reproductive system. Our data also suggest that ovarian toxicities following chronic exposure to TCDD result from downstream effects of AHR-mediated signal transduction pathways, or from feedback reactions to cellular changes induced by TCDD. Further examination of the relationships among altered structural integrity, glucose and lipid metabolism, immune response, and regulation of transcription in response to ovarian toxicity will better clarify the mechanisms by which TCDD exerts its reproductive toxicity, particularly its low-dose, long-term effects.

Acknowledgments: We gratefully acknowledge Lisa Meyer for her technical assistance. Research was supported in part by: USEPA (TKH, GRO MA916290), UWM Institute of Environmental Health (TKH/RJH), Greater Milwaukee Foundation Shaw Scientist Award (MJC), National Institute of Biomedical Imaging and Bioengineering (MJH, R01EB001421), and the UWM Marine and Freshwater Biomedical Center (P30ES004184).

Publisher's Disclaimer: This is a PDF file of an unedited manuscript that has been accepted for publication. As a service to our customers we are providing this early version of the manuscript. The manuscript will undergo copyediting, typesetting, and review of the resulting proof before it is published in its final citable form. Please note that during the production process errors may be

Reproductive Toxicology, Vol. 25, No. 1 (January 2008): pg. 47-57. DOI. This article is @ Elsevier and permission has been granted for this version to appear in e-Publications@Marquette. Elsevier does not grant permission for this article to be further copied/distributed or hosted elsewhere without the express permission from Elsevier. 
NOT THE PUBLISHED VERSION; this is the author's final, peer-reviewed manuscript. The published version may be accessed by following the link in the citation at the bottom of the page.

discovered which could affect the content, and all legal disclaimers that apply to the journal pertain.

\section{Reference List}

1. Peterson RE, Theobald HM, Kimmel GL. Developmental and reproductive toxicity of dioxins and related compounds: cross-species comparisons. Crit Rev Toxicol. 1993;23:283-335.

2. Walker MK, Peterson RE. Toxicity of polychlorinated dibenzo-p-dioxins, dibenzofurans, and biphenyls during fish early development. In: Colborn T, Clement C, editors. Chemically Induced Alterations in Sexual and Functional Development: The Wildlife/Human Connection. Princeton, New Jersey: Princeton Scientific Publishing, Co., Inc.; 1992. pp. 195-202.

3. Pocar P, Fischer B, Klonisch T, Hombach-Klonisch S. Molecular interactions of the aryl hydrocarbon receptor and its biological and toxicological relevance for reproduction. Reproduction. 2005;129:379-89.

4. King Heiden T, Carvan MJ, III, Hutz RJ. Inhibition of Follicular Development, Vitellogenesis, and Serum 17 \{beta\}-Estradiol Concentrations in Zebrafish Following Chronic, Sublethal Dietary Exposure to 2,3,7,8-Tetrachlorodibenzo-p-Dioxin. Toxicol Sci. 2006;90:490-9.

5. Pocar P, Brevini TA, Fischer B, Gandolfi F. The impact of endocrine disruptors on oocyte competence. Reproduction. 2003;125:313-25.

6. Roby KF. Alterations in follicle development, steroidogenesis, and gonadotropin receptor binding in a model of ovulatory blockade. Endocrinology. 2001;142:2328-35.

7. Munkittrick KR, Portt CB, Van der Kraak GJ, Smith IR, Rokosh DA. Impact of bleached kraft mill effluent on population characteristics, liver MFO activity, and serum steroid levels of a Lake Superior white sucker (Catostomus commersoni) population. Can J Fish Aquat Sci. 1991;48:1371-80.

8. Schmidt JV, Bradfield CA. Ah receptor signaling pathways. Annu Rev Cell Dev Biol. 1996;12:55-89.

9. Safe S, Krishnan V. Cellular and molecular biology of aryl hydrocarbon (Ah) receptor-mediated gene expression. Arch Toxicol Suppl. 1995;17:99115.

10. Denison DL, Gottlieb MB, Whitlock JP. The DNA recognition site for the dioxin-Ah receptor complex. Nucleotide sequence and functional analysis. J Biol Chem. 1988;263:17221-4.

11. Krishnan V, Porter W, Santostefano M, Wang X, Safe S. Molecular mechanism of inhibition of estrogen-induced cathepsin $D$ gene

Reproductive Toxicology, Vol. 25, No. 1 (January 2008): pg. 47-57. DOI. This article is @ Elsevier and permission has been granted for this version to appear in e-Publications@Marquette. Elsevier does not grant permission for this article to be further copied/distributed or hosted elsewhere without the express permission from Elsevier. 
NOT THE PUBLISHED VERSION; this is the author's final, peer-reviewed manuscript. The published version may be accessed by following the link in the citation at the bottom of the page.

expression by 2,3,7,8-tetrachlorodibenzo-p-dioxin (TCDD) in MCF-7 cells. Mol Cell Biol. 1995;15:6710-9.

12. Van der Kraak GJ, Munkittrick KR, McMaster ME, Portt CB, Chang JP. Exposure to bleached kraft pulp mill effluent disrupts the pituitarygonadal axis of white sucker at multiple sites. Toxicol Appl Pharmacol. 1992;115:224-33.

13. Gagnon MM, Dodson JJ, Hodson PV. Ability of BKME (bleached kraft mill effluent) exposed white suckers (Catostomus commersoni) to synthesize steroid hormones. Comp Biochem Physiol C. 1994; 107:265-73.

14. Hutz RJ. Reproductive endocrine disruption by environmental xenobiotics that modulate the estrogen-signaling pathway, particularly tetrachlorodibenzo-p-dioxin (TCDD) Journal of Reproduction and Development. 1999;45:1-12.

15. Son DS, Ushinohama K, Gao X, et al. 2,3,7,8-Tetrachlorodibenzo-p-dioxin (TCDD) blocks ovulation by a direct action on the ovary without alteration of ovarian steroidogenesis: lack of a direct effect on ovarian granulosa and thecal-interstitial cell steroidogenesis in vitro. Reprod Toxicol. 1999;13:521-30.

16. Trewin AL, Woller MJ, Wimpee BA, Conley LK, Baldridge MG, Hutz RJ. Short-Term Hormone Release from Adult Female Rat Hypothalamic and Pituitary Explants is not Altered by 2,3,7,8-Tetrachlorodibenzo-pdioxin. J Reprod Dev. 2007

17. Salisbury TB, Marcinkiewicz JL. In utero and lactational exposure to 2,3,7,8-tetrachlorodibenzo-p-dioxin and 2,3,4,7,8pentachlorodibenzofuran reduces growth and disrupts reproductive parameters in female rats. Biol Reprod. 2002;66:1621-6.

18. Safe S, Wormke M, Samudio I. Mechanisms of inhibitory aryl hydrocarbon receptor-estrogen receptor crosstalk in human breast cancer cells. J Mammary Gland Biol Neoplasia. 2000;5:295-306.

19. Safe S, Wormke M. Inhibitory aryl hydrocarbon receptor-estrogen receptor alpha cross-talk and mechanisms of action. Chem Res Toxicol. 2003;16:807-16.

20. Ohtake F, Takeyama K, Matsumoto T, et al. Modulation of oestrogen receptor signalling by association with the activated dioxin receptor. Nature. 2003;423:545-50.

21. Safe S, Krishnan V. Chlorinated hydrocarbons: estrogens and antiestrogens. Toxicol Lett. 1995;82-83:731-6.

22. Lict $P$. Suitability of the mammalian model in comparative reproductive endocrinology. In: Ralph C, editor. Comparative Endocrinology: Developments and directions. New York: Alan R Liss, Inc; 1986. pp. 95-114.

Reproductive Toxicology, Vol. 25, No. 1 (January 2008): pg. 47-57. DOI. This article is @ Elsevier and permission has been granted for this version to appear in e-Publications@Marquette. Elsevier does not grant permission for this article to be further copied/distributed or hosted elsewhere without the express permission from Elsevier. 
23. Tanguay RL, Andreasen E, Walker MK, Peterson RE. Dioxin toxicity and aryl hydrocarbon receptor signaling in fish. 2003.

24. Hahn ME, Karchner SI, Shapiro MA, Perera SA. Molecular evolution of two vertebrate aryl hydrocarbon (dioxin) receptors (AHR1 and AHR2) and the PAS family. Proc Natl Acad Sci U S A. 1997;94:13743-8.

25. Carvan MJ, III, King Heiden T, Tomasiawicz H. The Utility of Zebrafish as a Model for Toxicological Research. In: Mommsen TP, Moon TW, editors. Biochemistry and Molecular Biology of Fishes. Vol. 6. Elsevier BV; 2005. pp. 3-41.

26. Teraoka $H$, Dong $W$, Hiraga $T$. Zebrafish as a novel experimental model for developmental toxicology. Congenit Anom (Kyoto) 2003;43:12332.

27. Spitsbergen JM, Kent ML. The state of the art of the zebrafish model for toxicology and toxicologic pathology research--advantages and current limitations. Toxicol Pathol. 2003;31 (Suppl):62-87.

28. King Heiden T, Hutz RJ, Carvan MJ., III Accumulation, Tissue Distribution, and Maternal Transfer of Dietary 2,3,7,8,-Tetrachlorodibenzo-p-Dioxin: Impacts on Reproductive Success of Zebrafish. Toxicol Sci. 2005;87:497-507.

29. Pfaffl MW. A new mathematical model for relative quantification in realtime RT-PCR. Nucleic Acids Res. 2001;29:e45.

30. Brazma A, Hingamp P, Quackenbush J, et al. Minimum information about a microarray experiment (MIAME)-toward standards for microarray data. Nat Genet. 2001;29:365-71.

31. Kendziorski CM, Zhang $\mathrm{Y}$, Lan $\mathrm{H}$, Attie AD. The efficiency of pooling mRNA in microarray experiments. Biostatistics. 2003;4:465-77.

32. Shih JH, Michalowska AM, Dobbin K, Ye Y, Qiu TH, Green JE. Effects of pooling mRNA in microarray class comparisons. Bioinformatics. 2004;20:3318-25.

33. Zhang W, Carriquiry A, Nettleton D, Dekkers JC. Pooling mRNA in Microarray Experiments and its Effect on Power. Bioinformatics. 2007

34. Gentleman RC, Carey VJ, Bates DM, et al. Bioconductor: open software development for computational biology and bioinformatics. Genome Biol. 2004; 5:R80.

35. Eisen MB, Spellman PT, Brown PO, Botstein D. Cluster analysis and display of genome-wide expression patterns. Proc Natl Acad Sci U S A. 1998; $95: 14863-8$.

36. Karolchik D, Hinrichs AS, Furey TS, et al. The UCSC Table Browser data retrieval tool. Nucleic Acids Res. 2004;32:D493-D496.

37. Lenhard B, Wasserman WW. TFBS: Computational framework for transcription factor binding site analysis. Bioinformatics. 2002;18:1135-6.

Reproductive Toxicology, Vol. 25, No. 1 (January 2008): pg. 47-57. DOI. This article is @ Elsevier and permission has been granted for this version to appear in e-Publications@Marquette. Elsevier does not grant permission for this article to be further copied/distributed or hosted elsewhere without the express permission from Elsevier 
NOT THE PUBLISHED VERSION; this is the author's final, peer-reviewed manuscript. The published version may be

accessed by following the link in the citation at the bottom of the page.

38. Sandelin A, Alkema W, Engstrom P, Wasserman WW, Lenhard B. JASPAR: an open-access database for eukaryotic transcription factor binding profiles. Nucleic Acids Res. 2004;32:D91-D94.

39. Matys V, Fricke $E$, Geffers R, et al. TRANSFAC: transcriptional regulation, from patterns to profiles. Nucleic Acids Res. 2003;31:374-8.

40. Munkittrick KR, McMaster ME, McCarthy LH, Servos MR, Van der Kraak GJ. An overview of recent studies on the potential of pulp-mill effluents to alter reproductive parameters in fish. J Toxicol Environ Health B Crit Rev. $1998 ; 1: 347-71$.

41. Ge W. Intrafollicular paracrine communication in the zebrafish ovary: the state of the art of an emerging model for the study of vertebrate folliculogenesis. Mol Cell Endocrinol. 2005;237:1-10.

42. Kwok HF, So WK, Wang Y, Ge W. Zebrafish gonadotropins and their receptors: I. Cloning and characterization of zebrafish folliclestimulating hormone and luteinizing hormone receptors--evidence for their distinct functions in follicle development. Biol Reprod. 2005;72:1370-81.

43. So WK, Kwok HF, Ge W. Zebrafish gonadotropins and their receptors: II. Cloning and characterization of zebrafish follicle-stimulating hormone and luteinizing hormone subunits--their spatial-temporal expression patterns and receptor specificity. Biol Reprod. 2005;72:1382-96.

44. Wu T, Patel H, Mukai S, et al. Activin, inhibin, and follistatin in zebrafish ovary: expression and role in oocyte maturation. Biol Reprod. 2000;62:1585-92.

45. Pang Y, Ge W. Gonadotropin and activin enhance maturational competence of oocytes in the zebrafish (Danio rerio) Biol Reprod. 2002;66:259-65.

46. Hirakawa T, Minegishi T, Abe K, et al. Effect of 2,3,7,8tetrachlorodibenzo-p-dioxin on the expression of follicle-stimulating hormone receptors during cell differentiation in cultured granulosa cells. Endocrinology. 2000;141:1470-6.

47. Hirakawa $T$, Minegishi $T$, Abe K, Kishi H, Ibuki $Y$, Miyamoto K. Effect of 2,3,7,8-tetrachlorodibenzo-p-dioxin on the expression of luteinizing hormone receptors during cell differentiation in cultured granulosa cells. Arch Biochem Biophys. 2000;375:371-6.

48. Petroff BK, Roby KF, Gao X, et al. A review of mechanisms controlling ovulation with implications for the anovulatory effects of polychlorinated dibenzo-p-dioxins in rodents. Toxicology. 2001;158:91-107.

49. Fukuzawa NH, Ohsako S, Wu Q, et al. Testicular cytochrome P450scc and LHR as possible targets of 2,3,7,8-tetrachlorodibenzo-p-dioxin (TCDD) in the mouse. Mol Cell Endocrinol. 2004;221:87-96.

Reproductive Toxicology, Vol. 25, No. 1 (January 2008): pg. 47-57. DOI. This article is (c) Elsevier and permission has been granted for this version to appear in e-Publications@Marquette. Elsevier does not grant permission for this article to be further copied/distributed or hosted elsewhere without the express permission from Elsevier. 
NOT THE PUBLISHED VERSION; this is the author's final, peer-reviewed manuscript. The published version may be

accessed by following the link in the citation at the bottom of the page.

50. Petroff BK, Gao X, Rozman KK, Terranova PF. Interaction of estradiol and 2,3,7,8-tetrachlorodibenzo-p-dioxin (TCDD) in an ovulation model: evidence for systemic potentiation and local ovarian effects. Reprod Toxicol. 2000;14:247-55.

51. Baba T, Mimura J, Nakamura N, et al. Intrinsic function of the aryl hydrocarbon (dioxin) receptor as a key factor in female reproduction. Mol Cell Biol. 2005;25:10040-51.

52. Moran FM, VandeVoort CA, Overstreet JW, Lasley BL, Conley AJ. Molecular target of endocrine disruption in human luteinizing granulosa cells by 2,3,7,8-tetrachlorodibenzo-p-dioxin: inhibition of estradiol secretion due to decreased 17alpha-hydroxylase/17,20-lyase cytochrome P450 expression. Endocrinology. 2003;144:467-73.

53. Drenth HJ, Bouwman CA, Seinen W, van den BM. Effects of some persistent halogenated environmental contaminants on aromatase (CYP19) activity in the human choriocarcinoma cell line JEG-3. Toxicol Appl Pharmacol. 1998;148:50-5.

54. Myllymaki SA, Haavisto TE, Brokken LJ, Viluksela M, Toppari J, Paranko J. In utero and lactational exposure to TCDD; steroidogenic outcomes differ in male and female rat pups. Toxicol Sci. 2005;88:534-44.

55. Dasmahapatra AK, Wimpee BA, Trewin AL, Wimpee CF, Ghorai JK, Hutz RJ. Demonstration of 2,3,7,8-tetrachlorodibenzo-p-dioxin attenuation of P450 steroidogenic enzyme mRNAs in rat granulosa cell in vitro by competitive reverse transcriptase-polymerase chain reaction assay. Mol Cell Endocrinol. 2000;164:5-18.

56. Aluru N, Vijayan MM. Aryl hydrocarbon receptor activation impairs cortisol response to stress in rainbow trout by disrupting the rate-limiting steps in steroidogenesis. Endocrinology. 2006;147:1895-903.

57. Menuet A, Pellegrini E, Anglade I, et al. Molecular characterization of three estrogen receptor forms in zebrafish: binding characteristics, transactivation properties, and tissue distributions. Biol Reprod. 2002;66:1881-92.

58. Menuet A, Le Page Y, Torres O, Kern L, Kah O, Pakdel F. Analysis of the estrogen regulation of the zebrafish estrogen receptor (ER) reveals distinct effects of ERalpha, ERbeta1 and ERbeta2. J Mol Endocrinol. 2004;32:975-86.

59. Tian Y, Ke S, Thomas T, Meeker RJ, Gallo MA. Transcriptional suppression of estrogen receptor gene expression by 2,3,7,8-tetrachlorodibenzo-pdioxin (TCDD) J Steroid Biochem Mol Biol. 1998;67:17-24.

60. Tian Y, Ke S, Thomas T, Meeker RJ, Gallo MA. Regulation of estrogen receptor mRNA by 2,3,7,8-tetrachlorodibenzo-p-dioxin as measured by competitive RT-PCR. J Biochem Mol Toxicol. 1998;12:71-7.

61. Wormke M, Stoner M, Saville B, Safe S. Crosstalk between estrogen receptor alpha and the aryl hydrocarbon receptor in breast cancer cells

Reproductive Toxicology, Vol. 25, No. 1 (January 2008): pg. 47-57. DOI. This article is @ Elsevier and permission has been granted for this version to appear in e-Publications@Marquette. Elsevier does not grant permission for this article to be further copied/distributed or hosted elsewhere without the express permission from Elsevier. 
NOT THE PUBLISHED VERSION; this is the author's final, peer-reviewed manuscript. The published version may be accessed by following the link in the citation at the bottom of the page.

involves unidirectional activation of proteasomes. FEBS Lett. 2000;478:109-12.

62. DeVito MJ, Thomas T, Martin E, Umbreit TH, Gallo MA. Antiestrogenic action of 2,3,7,8-tetrachlorodibenzo-p-dioxin: tissue-specific regulation of estrogen receptor in CD1 mice. Toxicol Appl Pharmacol. 1992;113:284-92.

63. Wang X, Porter W, Krishnan V, Narasimhan TR, Safe S. Mechanism of 2,3,7,8-tetrachlorodibenzo-p-dioxin (TCDD)-mediated decrease of the nuclear estrogen receptor in MCF-7 human breast cancer cells. Mol Cell Endocrinol. 1993;96:159-66.

64. Tijet N, Boutros PC, Moffat ID, Okey AB, Tuomisto J, Pohjanvirta R. Aryl hydrocarbon receptor regulates distinct dioxin-dependent and dioxinindependent gene batteries. Mol Pharmacol. 2006;69:140-53.

65. Wang Y, Ge W. Developmental profiles of activin betaA, betaB, and follistatin expression in the zebrafish ovary: evidence for their differential roles during sexual maturation and ovulatory cycle. Biol Reprod. 2004;71:2056-64.

66. Janz DM, Van Der Kraak G. Suppression of apoptosis by gonadotropin, 17beta-estradiol, and epidermal growth factor in rainbow trout preovulatory ovarian follicles. Gen Comp Endocrinol. 1997;105:18693.

67. Pati D, Balshaw K, Grinwich DL, Hollenberg MD, Habibi HR. Epidermal growth factor receptor binding and biological activity in the ovary of goldfish, Carassius auratus. Am J Physiol. 1996;270:R1065-R1072.

68. Andreasen EA, Mathew LK, Tanguay RL. Regenerative Growth is Impacted by TCDD: Gene Expression Analysis Reveals Extracellular Matrix Modulation. Toxicol Sci. 2006

69. Volz DC, Hinton DE, Law JM, Kullman SW. Dynamic Gene Expression Changes Precede Dioxin-Induced Liver Pathogenesis in Medaka Fish. Toxicol Sci. 2005

70. Kajihara M, Kawauchi S, Kobayashi M, Ogino H, Takahashi S, Yasuda K. Isolation, characterization, and expression analysis of zebrafish large Mafs. J Biochem (Tokyo) 2001;129:139-46.

71. Li MA, Alls JD, Avancini RM, Koo K, Godt D. The large Maf factor Traffic Jam controls gonad morphogenesis in Drosophila. Nat Cell Biol. 2003;5:994-1000.

72. Yu JH, Guo J, Guo J, Zeng FX, Tang GH. The inhibitory effect and its mechanism of transferrin on $\mathrm{FSH}$-induced differentiation of granulosa cells. Sheng Li Xue Bao. 1994;46:209-16.

73. $\mathrm{Yu} \mathrm{JH}$, Findlay JK. An inhibitory effect of transferrin on differentiation of rat granulosa cells in vitro. Endocrinology. 1991;128:1841-8.

Reproductive Toxicology, Vol. 25, No. 1 (January 2008): pg. 47-57. DOI. This article is @ Elsevier and permission has been granted for this version to appear in e-Publications@Marquette. Elsevier does not grant permission for this article to be further copied/distributed or hosted elsewhere without the express permission from Elsevier. 
NOT THE PUBLISHED VERSION; this is the author's final, peer-reviewed manuscript. The published version may be

accessed by following the link in the citation at the bottom of the page.

74. Handley-Goldstone HM, Grow MW, Stegeman JJ. Cardiovascular gene expression profiles of dioxin exposure in zebrafish embryos. Toxicol Sci. 2005;85:683-93.

75. Carney SA, Chen J, Burns CG, Xiong KM, Peterson RE, Heideman W. AHR Activation Produces Heart-Specific Transcriptional and Toxic Responses in Developing Zebrafish. Mol Pharmacol. 2006

76. Volz DC, Bencic DC, Hinton DE, Law JM, Kullman SW. 2,3,7,8-

Tetrachlorodibenzo-p-dioxin (TCDD) induces organ- specific differential gene expression in male Japanese medaka (Oryzias latipes) Toxicol Sci. 2005;85:572-84.

77. Mishra A, Joy KP. Ovarian monosaccharides (glucose and fructose): hormonal effects and their role in final oocyte maturation and egg quality in catfish Heteropneustes fossilis, Bloch. Indian J Exp Biol. 2004;42:1084-90.

78. Bornstein SR, Rutkowski H, Vrezas I. Cytokines and steroidogenesis. Mol Cell Endocrinol. 2004;215:135-41.

79. von Schalburg KR, Rise ML, Brown GD, Davidson WS, Koop BF. A comprehensive survey of the genes involved in maturation and development of the rainbow trout ovary. Biol Reprod. 2005;72:68799.

80. Puga A, Maier A, Medvedovic $M$. The transcriptional signature of dioxin in human hepatoma HepG2 cells. Biochem Pharmacol. 2000;60:1129-42.

81. Pande K, Moran SM, Bradfield CA. Aspects of dioxin toxicity are mediated by interleukin 1-like cytokines. Mol Pharmacol. 2005;67:1393-8.

82. Chen I, Hsieh T, Thomas T, Safe S. Identification of estrogen-induced genes downregulated by AhR agonists in MCF-7 breast cancer cells using suppression subtractive hybridization. Gene. 2001;262:207-14.

83. Kharat I, Saatcioglu F. Antiestrogenic effects of $2,3,7,8-$ tetrachlorodibenzo-p-dioxin are mediated by direct transcriptional interference with the liganded estrogen receptor. Cross-talk between aryl hydrocarbon- and estrogen-mediated signaling. J Biol Chem. $1996 ; 271: 10533-7$.

84. Cheshenko K, Brion F, Le Page $Y$, et al. Expression of Zebra Fish Aromatase cyp19a and cyp19b Genes in Response to the Ligands of Estrogen Receptor and Aryl Hydrocarbon Receptor. Toxicol Sci. 2007;96:255-67.

85. Bemanian V, Male R, Goksoyr A. The aryl hydrocarbon receptor-mediated disruption of vitellogenin synthesis in the fish liver: Cross-talk between AHR-and ERalpha-signalling pathways. Comp Hepatol. 2004;3:2.

86. Tong SK, Chung BC. Analysis of zebrafish cyp19 promoters. J Steroid Biochem Mol Biol. 2003;86:381-6.

Reproductive Toxicology, Vol. 25, No. 1 (January 2008): pg. 47-57. DOI. This article is @ Elsevier and permission has been granted for this version to appear in e-Publications@Marquette. Elsevier does not grant permission for this article to be further copied/distributed or hosted elsewhere without the express permission from Elsevier 
Supplementary Table 1. Summary of cluster analysis and transcription factor analysis (unique transcripts)

\begin{tabular}{|c|c|c|c|c|c|c|c|c|}
\hline Cluster & Affy ID & Symbol & Description & 10ppb & 40ppb & 100ppb & AHRE & ERE \\
\hline 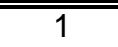 & "Dr.8215.1.A1_at & sox21a & "SRY-box containing gene 21a & -2.33 & -1.32 & -1.00 & $\overline{\mathrm{Y}}$ & $\bar{Y} \mathrm{Y}$ \\
\hline 1 & Dr.7110.1.S1_at & zgc:77614 & zgc:77059 & -3.04 & -2.42 & -7.29 & Y & Y \\
\hline 1 & Dr.6147.1.A1_at & zgc:77150 & zgc:76913 & -2.95 & -1.70 & -4.17 & $\mathrm{Y}$ & $\mathrm{Y}$ \\
\hline 1 & Dr.2978.1.S1_at & vg3 & vitellogenin 3 , phosvitinless & -6.65 & -1.67 & -1.79 & Y & Y \\
\hline 1 & Dr.25593.2.Ā1_at & wu:fa56g11 & wu:fa56g11 & -3.90 & -1.03 & -1.20 & Y & Y \\
\hline 1 & Dr.25285.1.S1_at & zgc:73223 & zgc:73223 & -2.47 & -1.15 & -1.11 & Y & Y \\
\hline 1 & Dr.25009.4.A1_at & wu:fb59c05 & $\begin{array}{l}\text { wu:fb59c05 } \\
\text { Kreisler (mouse) maf-related leucine zipper }\end{array}$ & -7.93 & -1.73 & -2.05 & Y & Y \\
\hline 1 & Dr.23470.1.S1_s_at & $\mathrm{krml} 2.2$ & homolog 2.2 & -3.67 & -1.02 & -2.68 & $\mathrm{Y}$ & Y \\
\hline 1 & Dr.18893.1.S1_at & im:7138745 & im:7138745 & -2.73 & -1.06 & 1.01 & $\mathrm{Y}$ & Y \\
\hline 1 & Dr.16681.1.A1_at & & NA & -2.17 & 1.07 & 1.47 & $\mathrm{Y}$ & Y \\
\hline 1 & Dr.11971.1.A1_at & zgc:92317 & zgc:91877 & -2.34 & -1.06 & -1.46 & $\mathrm{Y}$ & Y \\
\hline 1 & Dr.921.1.A1_at & timeless & timeless homolog (Drosophila) & -2.92 & 1.88 & 1.07 & $\mathrm{Y}$ & \\
\hline 1 & Dr.8090.1.Ā1_at & ndrg1l & $\begin{array}{l}\mathrm{N} \text {-myc downstream regulated gene } 1 \text {, like } \\
\text { procollagen-lysine } 1,2 \text {-oxoglutarate } 5 \text { - }\end{array}$ & -3.58 & 1.33 & -1.93 & $\mathrm{Y}$ & \\
\hline 1 & Dr.7688.1.A1_at & plod1 & dioxygenase 1 & -2.24 & 1.00 & -1.43 & Y & \\
\hline 1 & Dr.25774.1.S1_at & & NA & -3.06 & -1.59 & -1.94 & Y & \\
\hline 1 & Dr.25498.1.S1_at & bzw1 & basic leucine zipper and W2 domains 1 & -2.15 & 1.43 & 1.18 & Y & \\
\hline 1 & Dr.25009.6.A1_a_at & $\operatorname{vg} 1$ & vitellogenin 1 & -7.38 & -1.62 & -1.83 & $\mathrm{Y}$ & \\
\hline 1 & Dr.24920.1.S1_at & & NA & -2.56 & -1.13 & -1.36 & Y & \\
\hline 1 & Dr.24253.1.S1_at & stk3 & serine/threonine kinase 3 (STE20 homolog, & -3.84 & 1.86 & -1.83 & $\mathrm{Y}$ & \\
\hline 1 & Dr.20140.1.A1_at & $\mathrm{E} 2 \mathrm{~F} 4$ & NA & -2.60 & -1.64 & -1.50 & $\mathrm{Y}$ & \\
\hline 1 & Dr.1991.1.A1_s_at & zgc: 66417 & zgc:66417 & -7.09 & -1.19 & -5.92 & Y & \\
\hline 1 & Dr.195.1.S2_at & ck2a1 & casein kinase 2 alpha 1 & -3.29 & -1.04 & -1.86 & Y & \\
\hline 1 & Dr.18466.1.A1_at & & NA & -2.23 & 1.03 & -1.56 & Y & \\
\hline 1 & Dr.17776.1.A1_at & tph1l & tryptophan hydroxylase 1 , like & -2.64 & -1.53 & 1.19 & Y & \\
\hline 1 & Dr.17113.1.A1_a_at & ptena & phosphatase and tensin homolog $\mathrm{A}$ & -2.32 & 1.51 & 1.38 & Y & \\
\hline 1 & Dr.13994.2.A1_a_at & zgc:101086 & zgc:101086 & -2.37 & -1.20 & -1.67 & $\mathrm{Y}$ & \\
\hline 1 & Dr.13775.1.S1_at & & NA & -3.60 & -1.20 & -1.50 & Y & \\
\hline 1 & Dr.12714.1.A1_at & & NA & -5.65 & -1.56 & -1.01 & $\mathrm{Y}$ & \\
\hline 1 & Dr.10170.1.A1_at & & NA & -2.38 & -1.23 & -1.64 & Y & \\
\hline 1 & Dr.4412.13.A1_x_at & wu:fa56g11 & wu:fa56g11 & -2.92 & 1.16 & -1.13 & & Y \\
\hline 1 & Dr.16504.1.A1_at & & NA & -2.60 & -1.33 & -1.90 & & $\mathrm{Y}$ \\
\hline 1 & Dr.16380.1.A1_at & zgc:92068 & zgc:86738 & -2.45 & -1.26 & -1.18 & & $\mathrm{Y}$ \\
\hline 1 & Dr.4412.12.A1_at & wu:fa56g11 & wu:fa56g11 & -3.55 & -1.05 & -1.32 & & \\
\hline 1 & Dr.25593.1.S1_at & wu:fa56g11 & wu:fa56g11 & -2.80 & 1.30 & 1.06 & & \\
\hline 1 & Dr.13096.2.S1_at & zgc:86738 & zgc:77882 & -3.95 & 1.18 & -1.89 & & \\
\hline 2 & Dr.9682.1.A1_at & & NA & -4.11 & -4.33 & -3.91 & $Y$ & $Y$ \\
\hline
\end{tabular}


Supplementary Table 1 (cont.). Summary of cluster analysis and transcription factor analysis (unique transcripts)

\begin{tabular}{|c|c|c|c|c|c|c|c|c|}
\hline Cluster & Affy ID & Symbol & Description & 10ppb & 40ppb & 100ppb & AHRE & ERE \\
\hline 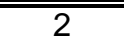 & Dr.956.1.S1_at & wu:fa92g05 & wu:fa92g05 & -1.17 & -1.36 & -2.22 & $\bar{Y}$ & $\overline{\bar{Y}}$ \\
\hline 2 & Dr.913.1.S1_at & zgc:63909 & zgc:63909 & -2.60 & -1.62 & -2.69 & $\mathrm{Y}$ & Y \\
\hline 2 & Dr.8723.1.s1__at & zgc:92903 & zgc:92068 & -1.21 & -1.61 & -3.77 & $\mathrm{Y}$ & Y \\
\hline 2 & Dr.7626.1.A1_at & zgc:73257 & zgc:73257 & -2.25 & -1.15 & -2.18 & $Y$ & Y \\
\hline 2 & Dr.7236.1.A1_at & wu:fc31g04 & wu:fc31g04 & -2.90 & -1.25 & -2.87 & $Y$ & Y \\
\hline 2 & Dr.5853.1.A1_at & zgc:77059 & zgc:76953 & -2.38 & -1.03 & -1.02 & $Y$ & Y \\
\hline 2 & Dr.4387.1.S1_at & krt4 & keratin 4 & -1.96 & -2.93 & -5.65 & $\mathrm{Y}$ & Y \\
\hline 2 & Dr.4249.1.S1_at & lipf & lipase, gastric & -2.06 & -1.85 & -2.30 & Y & Y \\
\hline 2 & Dr.4212.1.S1_at & Idha & lactate dehydrogenase A4 & -1.78 & -1.45 & -2.59 & $\mathrm{Y}$ & Y \\
\hline 2 & Dr.25155.1.sī_s_at & ba1 & ba1 globin & -1.11 & 1.22 & -2.50 & Y & Y \\
\hline 2 & Dr.24233.1.S1_at & $f n 1 b$ & fibronectin $1 b$ & -1.03 & -1.57 & -2.69 & $\mathrm{Y}$ & Y \\
\hline 2 & Dr.24122.1.A1_at & zgc:77882 & zgc:77614 & -2.49 & -2.05 & -2.06 & Y & Y \\
\hline 2 & Dr.20722.1.S1_at & zgc:56454 & zgc:56454 & -2.14 & -1.43 & -2.95 & Y & Y \\
\hline 2 & Dr.16206.1.A1_s_at & wu:fo83d04 & NA & 1.26 & 1.65 & 1.23 & $\mathrm{Y}$ & Y \\
\hline 2 & Dr.1619.1.A1_at & wu:fb83a09 & wu:fb83a09 & -3.44 & -1.98 & -2.03 & $\mathrm{Y}$ & Y \\
\hline 2 & Dr.15054.1.s1_at & $\mathrm{rbp} 2 \mathrm{a}$ & retinol binding protein $2 \mathrm{a}$, cellular & -2.98 & -2.39 & -4.54 & $\mathrm{Y}$ & Y \\
\hline 2 & Dr.13689.1.S1_at & zgc:66449 & zgc:66449 & -2.65 & -1.76 & -1.90 & $\mathrm{Y}$ & Y \\
\hline 2 & Dr.1260.1.S1_at & anxa2a & annexin $\mathrm{A} 2 \mathrm{a}$ & -2.42 & -2.92 & -4.84 & $\mathrm{Y}$ & Y \\
\hline 2 & Dr.12575.1.S1_at & dcp1a & decapping enzyme & -1.12 & -1.10 & -2.16 & $\mathrm{Y}$ & Y \\
\hline 2 & Dr.10326.1.S1_at & junb & jun B proto-oncogene & -2.21 & -1.88 & -4.39 & $\mathrm{Y}$ & $\mathrm{Y}$ \\
\hline 2 & Dr.890.1.S1_at & zgc: 77517 & zgc:77150 & -2.69 & -1.18 & -1.53 & $\mathrm{Y}$ & \\
\hline 2 & Dr.8045.1.sī_at & prtfdc1 & phosphoribosyl transferase domain containing & -1.44 & -1.12 & -3.05 & $\mathrm{Y}$ & \\
\hline 2 & Dr.59.1.S1_at & anxala & annexin A1a & -2.20 & -3.36 & -7.06 & $\mathrm{Y}$ & \\
\hline 2 & Dr.3628.1.S̄1_at & rap1b & RAS related protein $1 b$ & -1.79 & -1.41 & -2.40 & $\mathrm{Y}$ & \\
\hline 2 & Dr.3512.1.A1_at & & NA & -1.86 & -1.34 & -2.26 & $\mathrm{Y}$ & \\
\hline 2 & Dr.3459.1.S1_at & $\operatorname{cd} 163$ & Cd63 antigen & -2.04 & -1.47 & -3.70 & $\mathrm{Y}$ & \\
\hline 2 & Dr.3180.1.A1_at & zgc:76913 & zgc:76908 & -1.84 & -2.40 & -2.50 & $\mathrm{Y}$ & \\
\hline 2 & Dr.2363.1.S1_at & tagln2 & $\begin{array}{l}\text { transgelin } 2 \\
\text { lectin, galactoside-binding, soluble, } 3 \text { (galectin }\end{array}$ & -2.93 & -2.27 & -2.21 & Y & \\
\hline 2 & Dr.20815.3.A1_at & |gals3| & 3)-like & -1.89 & -2.68 & -2.56 & $\mathrm{Y}$ & \\
\hline 2 & Dr.20277.1.A1_at & acta2 & actin, alpha 2 , smooth muscle, aorta & -1.83 & -1.27 & -2.65 & $\mathrm{Y}$ & \\
\hline 2 & Dr.18135.1.A1_at & wu:fl63a03 & wu:fl63a03 & -1.07 & 1.07 & -4.01 & $\mathrm{Y}$ & \\
\hline 2 & Dr.17850.1.S1_at & zgc:56592 & zgc:56592 & -2.61 & -2.05 & -3.41 & $\mathrm{Y}$ & \\
\hline 2 & Dr.15930.1.A1_at & & NA & -1.51 & -1.03 & -2.18 & $\mathrm{Y}$ & \\
\hline 2 & Dr.15856.1.A1_at & & NA & -2.66 & -1.10 & -2.12 & $\mathrm{Y}$ & \\
\hline 2 & Dr.14748.1.S1_at & zgc:63985 & zgc:63985 & -1.54 & -1.52 & -2.65 & $\mathrm{Y}$ & \\
\hline 2 & Dr.14041.1.S1_at & zgc:103597 & zgc:103597 & -2.65 & -1.91 & -1.97 & $\mathrm{Y}$ & \\
\hline 2 & Dr.1377.1.A1_at & col1a2 & collagen, type I, alpha 2 & -2.47 & -1.44 & -2.56 & $\mathrm{Y}$ & \\
\hline
\end{tabular}


Supplementary Table 1 (cont.). Summary of cluster analysis and transcription factor analysis (unique transcripts)

\begin{tabular}{|c|c|c|c|c|c|c|c|c|}
\hline Cluster & Affy ID & Symbol & Description & 10ppb & 40ppb & 100ppb & AHRE & ERE \\
\hline 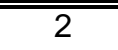 & "Dr.1372.1.S1_at & krt18 & "keratin 18 & -1.43 & -1.76 & -3.09 & $\overline{\mathrm{Y}}$ & \\
\hline 2 & Dr.1361.1.S1_at & wu:fa98f12 & wu:fa98f12 & -1.91 & -2.41 & -4.90 & $\mathrm{Y}$ & \\
\hline 2 & Dr.1282.1.S1_at & krt8 & keratin 8 & -1.45 & -1.89 & -3.02 & Y & \\
\hline 2 & Dr.1246.1.S1_at & apoeb & apolipoprotein Eb & -1.83 & -1.09 & -4.12 & $\mathrm{Y}$ & \\
\hline 2 & Dr.12106.1.sī_at & $|z t f| 1$ & leucine zipper transcription factor-like 1 & -1.12 & -1.12 & -2.33 & Y & \\
\hline 2 & Dr.1201.1.S1_at & zgc:56142 & zgc:56142 & -2.46 & -1.92 & -3.71 & Y & \\
\hline 2 & Dr.11609.1.S1_at & LOC402847 & & -1.62 & -1.54 & -2.86 & $\mathrm{Y}$ & \\
\hline 2 & Dr.11348.1.S1_at & zgc:86724 & zgc:77517 & -3.04 & -2.42 & -7.29 & $\mathrm{Y}$ & \\
\hline 2 & Dr.10879.1.A1_at & wu:fe14f11 & wu:fe14f11 & -1.82 & -1.12 & -2.38 & $\mathrm{Y}$ & \\
\hline 2 & Dr.10314.1.S1_a_at & mmp13 & matrix metalloproteinase 13 & 1.20 & 1.60 & -2.51 & $\mathrm{Y}$ & \\
\hline 2 & AFFX-Dr-GAPDH-3_at & & & & & & $\mathrm{Y}$ & \\
\hline 2 & Dr.4048.1.S1_at & & NA & -2.15 & -1.26 & -3.36 & & Y \\
\hline 2 & Dr.2973.1.A1_at & & NA & -1.49 & -2.39 & -1.77 & & Y \\
\hline 2 & Dr.24341.1.S1_at & zgc:76953 & zgc:76908 & -1.54 & -1.10 & -2.39 & & Y \\
\hline 2 & Dr.1411.1.S1_at & hbaa1 & $\begin{array}{l}\text { hemoglobin alpha adult-1 } \\
\text { Kreisler (mouse) maf-related leucine zipper }\end{array}$ & -1.10 & 1.27 & -2.64 & & Y \\
\hline 2 & Dr.8198.1.A1_at & $\mathrm{krml} 2$ & homolog 2 & -2.59 & -1.19 & -2.41 & & \\
\hline 2 & Dr.5809.1.A1_at & wu:fb11g12 & wu:fb11g12 & -2.30 & -1.57 & -7.28 & & \\
\hline 2 & Dr.5513.1.S1_at & slc3a2 & solute carrier family 3 , member 2 & -2.35 & -1.68 & -3.05 & & \\
\hline 2 & Dr.23469.1.S1_s_at & wu:fj17g12 & wu:fj17g12 & -2.30 & -2.50 & -2.56 & & \\
\hline 2 & Dr.18880.1.A1_at & & NA & -2.05 & -1.26 & -2.13 & & \\
\hline 2 & Dr.16206.1.A1_at & wu:fo83d04 & wu:fo83d04 & -1.22 & -1.06 & -6.09 & & \\
\hline 2 & Dr.13510.1.S1_at & actr10 & actin-related protein 10 homolog (S. cerevisiae) & -1.80 & -1.23 & -2.29 & & \\
\hline 2 & Dr.12498.1.S1_at & rad1 & RAD1 homolog (S. pombe) & -1.10 & 1.13 & -2.16 & & \\
\hline 2 & Dr.1194.1.S1_at & zgc:76908 & zgc:76908 & -2.34 & -1.85 & -3.53 & & \\
\hline 3 & Dr.8390.1.S1_at & zgc:55420 & zgc:55420 & 3.84 & -1.12 & 1.13 & Y & Y \\
\hline 3 & Dr.728.4.S1_at & $\mathrm{fgb}$ & fibrinogen, $B$ beta polypeptide & 5.66 & 1.43 & 1.61 & Y & Y \\
\hline 3 & Dr.6550.1.A1_at & LOC407646 & & 3.05 & 1.42 & -1.09 & $\mathrm{Y}$ & $\mathrm{Y}$ \\
\hline 3 & Dr.4797.1.S1_at & & NA & 8.50 & -1.19 & 1.20 & $\mathrm{Y}$ & $\mathrm{Y}$ \\
\hline 3 & Dr.3581.1.S1_at & ctrb1 & chymotrypsinogen B1 & 2.58 & 1.84 & -2.41 & $\mathrm{Y}$ & $\mathrm{Y}$ \\
\hline 3 & Dr.3025.3.S1_at & wu:fa04f09 & wu:fa04f09 & 17.52 & 1.37 & 2.04 & $\mathrm{Y}$ & $\mathrm{Y}$ \\
\hline 3 & Dr.25379.1.S1_at & sb:cb37 & sb:cb37 & 5.19 & 1.73 & 1.80 & $\mathrm{Y}$ & $\mathrm{Y}$ \\
\hline 3 & Dr.2426.1.S1_at & zgc:66321 & $\begin{array}{l}\text { zgc: } 66321 \\
\text { serine (or cysteine) proteinase inhibitor, clade } A\end{array}$ & 5.00 & -1.34 & -1.29 & $\mathrm{Y}$ & $\mathrm{Y}$ \\
\hline 3 & Dr.1605.1.S1_at & serpina1 & (alpha-1 antiproteinase, antitrypsin), member 1 & 29.44 & 1.51 & 3.07 & Y & Y \\
\hline 3 & Dr.8587.1.A2_at & igfbp1 & insulin-like growth factor binding protein 1 & 5.58 & -1.38 & -1.47 & Y & \\
\hline 3 & Dr.5462.1.S1_at & $\mathrm{fgb}$ & fibrinogen, $\mathrm{B}$ beta polypeptide & 12.80 & -1.82 & -1.35 & Y & \\
\hline 3 & Dr.4907.1.S1_at & fgg & fibrinogen, gamma polypeptide & 12.96 & -1.66 & -1.12 & Y & \\
\hline
\end{tabular}


Supplementary Table 1 (cont.). Summary of cluster analysis and transcription factor analysis (unique transcripts)

\begin{tabular}{|c|c|c|c|c|c|c|c|c|}
\hline Cluster & Affy ID & Symbol & Description & 10ppb & 40ppb & 100ppb & AHRE & ERE \\
\hline 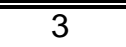 & "Dr.3613.1.S1_at & сcp & "ceruloplasmin & 24.49 & 1.06 & 2.16 & $\overline{\mathrm{Y}}$ & \\
\hline 3 & Dr.20270.1.S1_at & wu:fi20c07 & wu:fi20c07 & 12.95 & -1.13 & 1.33 & $\mathrm{Y}$ & \\
\hline 3 & Dr.1889.2.A1_āa_at & $\mathrm{tfa}$ & transferrin-a & 11.64 & -2.09 & -1.32 & $\mathrm{Y}$ & \\
\hline 3 & Dr.5562.1.S1_at & si:ch211-219i10.1 & si:ch211-219i10.1 & 16.55 & 1.24 & 1.12 & & \\
\hline 3 & Dr.1889.1.S1_at & $\mathrm{tfa}$ & transferrin-a & 9.49 & -2.25 & -1.42 & & \\
\hline 4 & Dr.7806.1.A1_at & & NA & -2.44 & 1.04 & 2.65 & Y & $\mathrm{Y}$ \\
\hline 4 & Dr.26469.1.sī_at & sp4 & sp4 transcription factor & 1.51 & 2.32 & 1.96 & $\mathrm{Y}$ & Y \\
\hline 4 & Dr.16524.1.S1_at & wu:fc12b04 & wu:fc12b04 & 1.85 & 2.39 & 2.05 & Y & $\mathrm{Y}$ \\
\hline 4 & Dr.13972.1.S1_at & zgc:64114 & zgc:64114 & 1.19 & 2.45 & 4.16 & Y & Y \\
\hline 4 & Dr.13182.1.A1_at & rab5b & RAB5B, member RAS oncogene family & 2.45 & 1.34 & 2.13 & Y & $\mathrm{Y}$ \\
\hline 4 & Dr.12833.1.A1_at & zgc:64213 & zgc:64213 & -1.62 & 1.18 & 3.28 & Y & $\mathrm{Y}$ \\
\hline 4 & Dr.1192.1.S1_at & wu:fb11h03 & wu:fb11h03 & -1.60 & -1.17 & 3.48 & $\mathrm{Y}$ & $\mathrm{Y}$ \\
\hline 4 & Dr.9478.1.S1_at & cyp1a & cytochrome P450, family 1 , subfamily A & 6.93 & 14.87 & 24.68 & Y & \\
\hline 4 & Dr.9450.1.A1_at & zgc:63724 & zgc:63724 & -1.46 & 1.74 & 3.59 & Y & \\
\hline 4 & Dr.19990.1.s̄̄_at & zgc:66101 & zgc:66101 & 1.57 & 1.55 & 2.43 & Y & \\
\hline 4 & Dr.19877.1.S1_at & zgc:66133 & zgc:66133 & 1.72 & 2.17 & 3.31 & Y & \\
\hline 4 & Dr.17662.1.S1_at & & NA & 1.88 & 3.38 & 1.62 & Y & \\
\hline 4 & Dr.15418.1.S1_at & wu:fc23f06 & wu:fc23f06 & 1.26 & 1.27 & 3.01 & $\mathrm{Y}$ & \\
\hline 4 & AFFX-Dr-acta1-5_x_at & & & & & & Y & \\
\hline 4 & Dr.24858.1.S1_at & zgc:110540 & zgc:110540 & 1.37 & 2.56 & 1.97 & & \\
\hline 4 & Dr.15555.1.S1_at & & NA & 1.48 & 2.58 & 2.72 & & \\
\hline 4 & Dr.1192.1.S1_āa_at & wu:fb11h03 & wu:fb11h03 & -2.96 & -1.56 & 2.95 & & \\
\hline 5 & Dr.26139.1.A $\overline{1} \_\bar{x} \_$at & NA & NA & 1.36 & 2.17 & -1.07 & Y & $\mathrm{Y}$ \\
\hline 5 & Dr.25378.1.A1_at & zgc:110540 & zgc:110540 & 1.64 & 2.32 & 1.48 & Y & $\mathrm{Y}$ \\
\hline 5 & Dr.24249.1.S1_at & zc3hdc5 & zinc finger $\mathrm{CCCH}$ type domain containing 5 & 1.60 & 1.54 & 1.02 & Y & Y \\
\hline 5 & Dr.5002.1.A1_at & NA & 然 & 1.74 & 2.33 & 1.78 & Y & \\
\hline 5 & Dr.25086.1.Ā1_at & NA & NA & 1.43 & 1.30 & 1.03 & Y & \\
\hline 5 & Dr.1368.7.S1_at & zgc:86724 & zgc:86724 & 1.26 & 1.38 & 1.30 & Y & \\
\hline
\end{tabular}




\begin{tabular}{|c|c|c|c|c|c|c|c|c|}
\hline $\begin{array}{l}\text { Supplementary Table } 2 . \\
\text { Gene ID }\end{array}$ & Transcript ID & Affy IDs & Symbol & Description & Start & End & Strand & Sequence \\
\hline$\overline{\text { ENSDARG00000000935 }}$ & ENSDART00000001053 & Dr.24249.1.S1_at & zc3hdc5 & zinc finger CCCH type domain containing 5 [Source:RefSeq_peptide;ACc:NP_956530] & -4874 & -4869 & 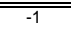 & agcgtg \\
\hline ENSDARG00000000935 & ENSDART00000001053 & Dr.24249.1.S1_at & zc3hdc5 & zinc finger $\mathrm{CCCH}$ type domain containing 5 [Source:RefSeq_peptide;Acc:NP_-956530] & -2211 & -2206 & -1 & tgcgtg \\
\hline ENSDARG00000000935 & ENSDART00000001053 & Dr.24249.1.S1_at & zc3hdc5 & zinc finger $\mathrm{CCCH}$ type domain containing 5 [Source:RefSeq_peptide;Acc:NP_-956530] & 210 & 215 & -1 & tgcgtg \\
\hline ENSDARG00000000935 & ENSDART00000001053 & Dr.24249.1.S1_at & zc3hdc5 & zinc finger $\mathrm{CCCH}$ type domain containing 5 [Source:RefSeq_peptide;Acc:NP_956530] & 748 & 753 & -1 & agcgtg \\
\hline ENSDARG00000001953 & ENSDART00000003247 & Dr.7626.1.A1_at,Dr.7626.2.S1_at & NP_998562.1 & zgc:73257 [Source:RefSeq_peptide;Acc:NP_998562] & -1683 & -1678 & -1 & $\operatorname{tgcgtg}$ \\
\hline $\begin{array}{l}\text { ENSDARG00000001953 } \\
\text { ENSDARG0000000153 }\end{array}$ & $\begin{array}{l}\text { ENSDART000000003247 } \\
\text { ENDART0000003247 }\end{array}$ & 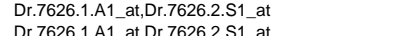 & $\begin{array}{l}\text { NP_-998562.1 } \\
\text { NP } 99852.1\end{array}$ & 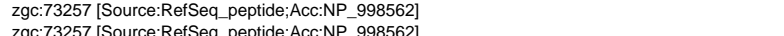 & $\begin{array}{l}-1087 \\
-862\end{array}$ & -1082 & $\begin{array}{c}-1 \\
-1\end{array}$ & $\begin{array}{l}\text { ggcgtg } \\
\text { agcta }\end{array}$ \\
\hline $\begin{array}{l}\text { ENSDARG00000001953 } \\
\text { ENSDARG00000001953 }\end{array}$ & $\begin{array}{l}\text { ENSDART0000000033247 } \\
\text { ENSDART0000003247 }\end{array}$ & 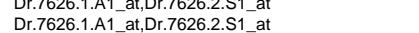 & NP-998562.1 & 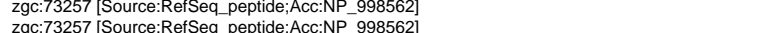 & $\begin{array}{l}-862 \\
-347\end{array}$ & $\begin{array}{l}-85 / 2 \\
-342\end{array}$ & $\begin{array}{c}-1 \\
1\end{array}$ & agcgtg \\
\hline ENSDARG00000002082 & ENSDART00000005010 & $\begin{array}{l}\text { D.r.r626.6.1.A1_at,Dr.79626.2.S1_at } \\
\text { Dr.195.1.S1_at,Dr.195.1.S2_at,Dr.195.1.S1_at }\end{array}$ & $\begin{array}{l}\mathrm{NP}-998562.1 \\
\mathrm{ck} 2 \mathrm{a} 1\end{array}$ & 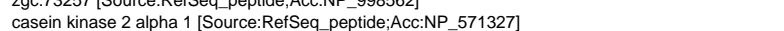 & $\begin{array}{l}-341 \\
-983\end{array}$ & -978 & 1 & agcatg \\
\hline ENSDARG00000002082 & ENSDART00000005010 & Dr.195.1.S1_at,Dr.195.1.S2_at,Dr.195.1.S1_at & ck2a1 & casein kinase 2 alpha 1 [Source:RefSeq peptide:Acc:NP 571327] & -941 & -936 & & cgcotg \\
\hline ENSDARG00000002082 & ENSDART00000005010 & Dr.195.1.S1 at,Dr.195.1.S2 at,Dr.195.1.S1 at & ck2a1 & casein kinase 2 alpha 1 [Source:RefSeq peptide;Acc:NP 571327] & -846 & -841 & -1 & tocotg \\
\hline ENSDARG00000002082 & ENSDART00000005010 & Dr.195.1.S1_at,Dr.195.1.S2_at,Dr.195.1.S1_at & ck2a1 & casein kinase 2 alpha 1 [Source:RefSeq_peptide;Acc:NP_571327] & -726 & -721 & 1 & gocgtg \\
\hline ENSDARG00000002082 & ENSDART00000005010 & Dr.195.1.S1_at,Dr.195.1.S2_at,Dr.195.1.S1_at & ck2a1 & casein kinase 2 alpha 1 [Source:RefSeq_peptide;Acc:NP_571327] & -553 & -548 & 1 & ggcgtg \\
\hline ENSDARG00000002667 & ENSDART00000003004 & Dr.23470.1.S1_s_at,Dr.8198.1.A1_at & $\mathrm{krml2.2}$ & Kreisler (mouse) maf-related leucine zipper homolog 2.2 [Source:RefSeq_peptide;Acc:NP_571917] & -248 & -243 & 1 & ggctgtg \\
\hline ENSDARG00000002667 & ENSDART00000003004 & Dr.23470.1.S1_s_at,Dr.8198.1.A1_at & $\mathrm{krm} 2.2$ & Kreisler (mouse) maf-related leucine zipper homolog 2.2 [Source:RefSeq_peptide;Acc:NP_571917] & -215 & -210 & 1 & agcgtg \\
\hline ENSDARG00000002667 & ENSDART00000003004 & Dr.23470.1.S1_s_at,Dr.8198.1.A1_at & $\mathrm{krml2.2}$ & Kreisler (mouse) maf-related leucine zipper homolog 2.2 [Source:RefSeq_peptide;Acc:NP_571917] & -248 & -243 & 1 & ggcgtg \\
\hline ENSDARG00000002667 & 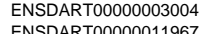 & Dr.23470.1.S1_s_at,Dr.8198.1.A1_at & krml2.2 & Kreisler (mouse) maf-related leucine zipper homolog 2.2 [Source:RefSeq_peptide;Acc:NP_571917] & -215 & -210 & 1 & agcgtg \\
\hline ENSDARG000000003216 & $\begin{array}{l}\text { ENSDART00000011967 } \\
\text { ENSART000001767 }\end{array}$ & $\begin{array}{ll}\text { Dr.1260.1.S1_at } \\
\text { Dr. at }\end{array}$ & & annexin A2a (anxa2a), mRNA [Source:RefSeq_dna;Acc:NM_181761] & -3672 & -3667 & -1 & agcgtg \\
\hline $\begin{array}{l}\text { ENSDARG000000003323 } \\
\text { ENSDARG0000000323 }\end{array}$ & $\begin{array}{l}\text { ENSDART000000017557 } \\
\text { ENDART0000017567 }\end{array}$ & $\begin{array}{l}\text { Dr.12575.1.S1_at } \\
\text { D.1255 }\end{array}$ & $\begin{array}{l}\text { dcppaa } \\
\text { dcppaa }\end{array}$ & 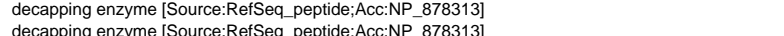 & $\begin{array}{l}-2628 \\
-2299\end{array}$ & $\begin{array}{l}-2623 \\
-2924\end{array}$ & $\begin{array}{l}-1 \\
-1\end{array}$ & $\begin{array}{l}\operatorname{tgcgtg} \\
\text { gactg }\end{array}$ \\
\hline $\begin{array}{l}\text { ENSDARG000000003333 } \\
\text { ENSDARG00000003323 }\end{array}$ & $\begin{array}{l}\text { ENSDART000000017567 } \\
\text { ENSDART0000017567 }\end{array}$ & $\begin{array}{l}\text { D.r.12575.1.S1_at } \\
\text { D.12575.1.S1 at }\end{array}$ & $\begin{array}{l}\text { dcppa } \\
\text { dcp1a }\end{array}$ & 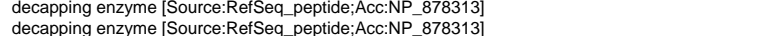 & $\begin{array}{l}-2299 \\
-170\end{array}$ & -2294 & $\begin{array}{c}-1 \\
1\end{array}$ & $\begin{array}{l}\text { ggctg } \\
\text { agctg }\end{array}$ \\
\hline ENSDARG00000004060 & ENSDART00000026017 & Dr.3180.1.A1_at at & zgc:76913 & Unknown (protein for MGC: 76913) [Source:RefSeq_peptide;Acc:NP 997844] & -1103 & -1098 & -1 & 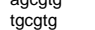 \\
\hline 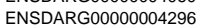 & 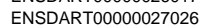 & Dr.2426.1.S1 at & zac: 66321 & 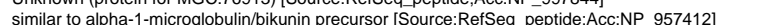 & -3749 & -3744 & 1 & 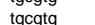 \\
\hline ENSDARG00000004296 & ENSDART00000027026 & Dr.2426.1.S1_at & zgc:66321 & similar to alpha-1-microglobulin/bikunin precursor [Source:RefSeq_peptide;Acc:NP_957412] & -3743 & -3738 & 1 & tgcgtg \\
\hline ENSDARG00000004296 & ENSDART00000027026 & Dr.2426.1.S1_at & zgc:66321 & similar to alpha-1-microglobulin/bikunin precursor [Source:RefSeq_peptide;Acc:NP_-957412] & -3737 & -3732 & 1 & tgcgtg \\
\hline ENSDARG00000004296 & ENSDART00000027026 & Dr.2426.1.S1_at at & zgc:66321 & similar to alpha-1-microglobulin/bikunin precursor [Source:RefSeq_peptide;Acc:NP_-957412] & -3725 & -3720 & 1 & tgcgtg \\
\hline ENSDARG00000004296 & ENSDART00000027026 & Dr.2426.1.S1_at & zgc:66321 & similar to alpha-1-microglobulin/bikunin precursor [Source:RefSeq_peptide;Acc:NP_957412] & 30 & 35 & 1 & tgcgtg \\
\hline ENSDARG00000005186 & ENSDART00000002285 & Dr.26469.1.S1_at & NP_956418.1 & sp4 transcription factor [Source:RefSeq_peptide;Acc:NP_956418] & -3691 & -3686 & 1 & tgcgtg \\
\hline ENSDARG00000005186 & ENSDART00000002285 & Dr.26469.1.S1_at & NP_956418.1 & sp4 transcription factor [Source:RefSeq_peptide;Acc:NP_956418] & -3596 & -3591 & 1 & agcgtg \\
\hline ENSDARG00000005186 & $\begin{array}{l}\text { ENSDART000000002285 } \\
\text { ENSART000000285 }\end{array}$ & $\begin{array}{l}\text { Dr.26469.1.S1_at } \\
\text { Dr.2649.1_1 at }\end{array}$ & NP_956418.1 & 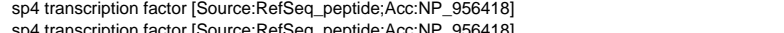 & -955 & -950 & -1 & $\operatorname{tgcgtg}$ \\
\hline $\begin{array}{l}\text { INSDARG000000005186 } \\
\text { ENSDARG00000005186 }\end{array}$ & $\begin{array}{l}\text { ENSDART000000002225 } \\
\text { ENSDART0000000285 }\end{array}$ & $\begin{array}{l}\text { Dr.26469.1.S1_at } \\
\text { D.r.26469.1S1 at }\end{array}$ & $\begin{array}{l}\text { NP_-956448.1 } \\
\text { NP } 956418.1\end{array}$ & 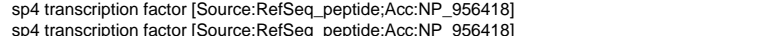 & $\begin{array}{l}-844 \\
-820\end{array}$ & $\begin{array}{l}-839 \\
-895\end{array}$ & $\begin{array}{c}1 \\
-1\end{array}$ & $\begin{array}{l}\text { ggcgttg } \\
\text { agctg }\end{array}$ \\
\hline $\begin{array}{l}\text { ENSDARG000000055186 } \\
\text { ENSDARG00000005186 }\end{array}$ & $\begin{array}{l}\text { ENSDART000000002285 } \\
\text { ENSDART0000002285 }\end{array}$ & $\begin{array}{l}\text { D.r.264666.1.S1_at } \\
\text { D.26469.1.S1 at }\end{array}$ & $\begin{array}{l}\text { NP-956448.1 } \\
\text { NP } 956418.1\end{array}$ & 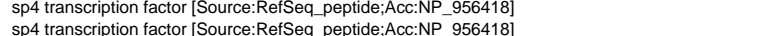 & $\begin{array}{l}-820 \\
-791\end{array}$ & $\begin{array}{l}-815 \\
-886\end{array}$ & $\begin{array}{c}-1 \\
1\end{array}$ & $\begin{array}{l}\text { agctg } \\
\text { ggctg }\end{array}$ \\
\hline ENSDARG00000005186 & ENSDART00000002285 & Dr.26469.1.S1 at & NP_956418.1 & sp4 transcription factor [Source:RefSeq_ peptide:Acc:NP 956418 ] & -532 & -527 & & gacgtg \\
\hline ENSDARG000000005186 & 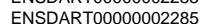 & D.26469.1.S1 at & $N^{-} 9564181$ & 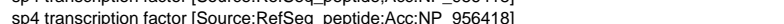 & -383 & -378 & -1 & 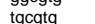 \\
\hline ENSDARG00000006074 & ENSDART00000002186 & Dr.15856.1.A1 at,Dr.13404.1.A1 at & NP_956234.1 & uridine monophosphate kinase [Source:RefSeq_peptide;:Acc:NP 956234] & -3203 & -3198 & 1 & ggcatg \\
\hline ENSDARG00000006526 & ENSDART00000017312 & Dr.24233.1.S1_at & $\mathrm{fn} 1 \overline{\mathrm{b}}$ & fibronectin 1b (fn1b), mRNA [Source:RefSeq_dna;Acc:NM_001013261] & -2209 & -2204 & 1 & ggcgtg \\
\hline ENSDARG00000006526 & ENSDART00000017312 & Dr.24233.1.S1_at & fn1b & fibronectin $1 \mathrm{~b}$ (fn1b), mRNA [Source:RefSeq_dna;ACc:NM__01013261] & -2163 & -2158 & -1 & ggctgtg \\
\hline ENSDARG00000006526 & ENSDART00000017312 & Dr.24233.1.S1_at & fn1b & fibronectin $1 \mathrm{~b}$ (fn1b), mRNA [Source:RefSeq_dna;ACc:NM__001013261] & -1504 & -1499 & -1 & tgcgtg \\
\hline ENSDARG00000006526 & ENSDART00000017312 & Dr.24233.1.S1_at & fn1b & fibronectin 1b (fn1b), mRNA [Source:RefSeq_dna;ACc:NM__001013261] & -4430 & -4425 & 1 & ggcgtg \\
\hline ENSDARG00000006526 & ENSDART00000017312 & Dr.24233.1.S1_at & fn1b & fibronectin 1b (fn1b), mRNA [Source:RefSeq_dna;ACc:NM__001013261] & -4387 & -4382 & -1 & agcgtg \\
\hline ENSDARG00000006526 & ENSDART00000023692 & Dr.24233.1.S1_at & fnib & fibronectin 1b (fn1b), mRNA [Source:RefSeq_dna;Acc:NM_001013261] & -2223 & -2218 & 1 & agcgtg \\
\hline $\begin{array}{l}\text { ENSDARG000000006526 } \\
\text { ENSDARG00000007219 }\end{array}$ & $\begin{array}{l}\text { ENSDART000000023692 } \\
\text { ENDART000000591 }\end{array}$ & $\begin{array}{l}\text { Dr.24233.1.S1_at } \\
\text { D.r.25774. S1 at }\end{array}$ & fn1b & fibronectin $1 \mathrm{~b}$ (fn1b), mRNA [Source:RefSeq_dna;ACc:NM__001013261] & $\begin{array}{l}-1263 \\
-2349\end{array}$ & $\begin{array}{l}-1258 \\
-2344\end{array}$ & $\underset{-1}{1}$ & $\begin{array}{l}\operatorname{tgcgtg} \\
\text { agctg }\end{array}$ \\
\hline ENSDARG00000007219 & ENSDART00000005791 & $\begin{array}{l}\text { lat.25774.1.S1_at } \\
\text { Dr.251 }\end{array}$ & & & -1147 & -1142 & -1 & 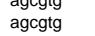 \\
\hline ENSDARG00000007219 & ENSDART00000005791 & Dr.25774.1.S1_at & & & -817 & -812 & -1 & agcgtg \\
\hline ENSDARG00000007481 & ENSDART00000058261 & Dr.5002.1.A1_at & & & -12 & -7 & 1 & cgcgtg \\
\hline ENSDARG00000008867 & ENSDART00000042250 & Dr.3628.1.S1_at & rap1b & RAS related protein $1 \mathrm{~b}$ [Source:RefSeq_peptide;Acc:NP_955827] & -3595 & -3590 & -1 & cgcgtg \\
\hline $\begin{array}{l}\text { ENSDARG000000008867 } \\
\text { ENSDARG000000867 }\end{array}$ & $\begin{array}{l}\text { ENSDART000000042250 } \\
\text { ENDART000004250 }\end{array}$ & $\begin{array}{l}\text { Dr.3628.1.S1_at } \\
\text { Dr.3628.S1 at }\end{array}$ & 党的1b & 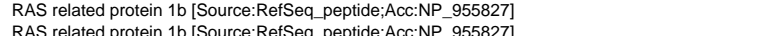 & $\begin{array}{l}-1533 \\
-234\end{array}$ & $\begin{array}{l}-1528 \\
-229\end{array}$ & $\begin{array}{c}-1 \\
-1\end{array}$ & $\begin{array}{l}\text { tgcgtg } \\
\text { accata }\end{array}$ \\
\hline ENSDARG00000008895 & $\begin{array}{l}\text { ENSDART000000042250 } \\
\text { ENSDART0000005048 }\end{array}$ & $\begin{array}{l}\text { D..3628.1.1.S1_at } \\
\text { Dr.1619.11.1619.1.A1_at,Dr.1619.1.A1_at }\end{array}$ & & RAS related protein $1 \mathrm{~b}$ [Source:RefSeq_peptide;ACc:NP__955827] & $\begin{array}{r}-234 \\
-1617\end{array}$ & $\begin{array}{r}-1612 \\
-269\end{array}$ & $\begin{array}{c}-1 \\
1\end{array}$ & $\begin{array}{l}\text { agcgge } \\
\text { cgcotg }\end{array}$ \\
\hline ENSDARG00000008969 & ENSDART00000011701 & Dr.5462.1.S1_at,Dr.5462.1.S1_at & fgb & fibrinogen, B beta polypeptide [Source:RefSeq_peptide;Acc:NP 997939$]$ & -3590 & -3585 & 1 & a actg \\
\hline ENSDARG00000008969 & ENSDART00000011701 & Dr.5462.1.S1__t,Dr.5462.1.S1_at & fgb & fibrinogen, B beta polypeptide [Source:RefSeg peptide:Acc:NP 997939 & -3263 & -3258 & 1 & gacta \\
\hline ENSDARG00000008969 & ENSDART00000011701 & Dr.5462.1.S1_at,Dr.5462.1.S1_at & fab & 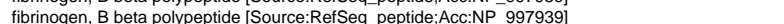 & -2312 & -2307 & -1 & $\begin{array}{l}\text { tgcotg } \\
\text { tgctg }\end{array}$ \\
\hline ENSDARG00000008969 & ENSDART00000011701 & Dr.5462.1.S1_at,Dr.5462.1.S1_at & fgb & fibrinogen, B beta polypeptide [Source:RefSeq_peptide;ACc:NP_-997939] & -2219 & -2214 & -1 & cgcgtg \\
\hline ENSDARG00000008969 & ENSDART00000041987 & Dr.5462.1.S1_at,Dr.5462.1.S1_at & fgb & & -311 & -306 & -1 & tgcgtg \\
\hline ENSDARG00000008969 & ENSDART00000041987 & Dr.5462.1.S1_at,Dr.5462.1.S1_at & fgb & fibrinogen, B beta polypeptide [Source:RefSeq_peptide;Acc:NP_-997939] & -178 & -173 & 1 & tgcgtg \\
\hline ENSDARG00000009483 & ENSDART00000009889 & Dr.25378.1.A1__at,Dr.24858.1. $\overline{\mathrm{S}} 1$ _at & zgc:110540 & deoxycytidine kinase [Source:RefSeq_peptide;Acc:NP_001014374] & -1352 & -1347 & -1 & cgcgtg \\
\hline ENSDARG00000009483 & ENSDART00000009889 & Dr.25378.1.A1_at,Dr.24858.1.S1_at & zgc:110540 & deoxycytidine kinase [Source:RefSeq_peptide;Acc:NP_001014374] & -1350 & -1345 & 1 & cgcgtg \\
\hline ENSDARG00000009483 & ENSDART00000009889 & Dr.25378.1.A1_at,Dr.24858.1.S1_at & zgc: 110540 & deoxycytidine kinase [Source:RefSeq_peptide;Acc:NP_001014374] & -1280 & -1275 & 1 & agcgtg \\
\hline $\begin{array}{l}\text { ENSDARG000000009483 } \\
\text { ENSDARG0000000983 }\end{array}$ & $\begin{array}{l}\text { ENSDART000000009889 } \\
\text { ENSDART0000000989 }\end{array}$ & $\begin{array}{l}\text { Dr.25378.1.A1_at,Dr.24858.1.S1_at } \\
\text { Dr 253781A1 atDr24858. S1 at }\end{array}$ & $\begin{array}{l}\text { zgc:110550 } \\
\text { zgc: } 110540\end{array}$ & 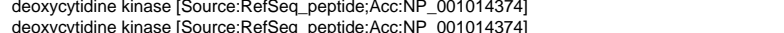 & -1352 & -1347 & -1 & cgcgtg \\
\hline $\begin{array}{l}\text { ENSDARG000000009483 } \\
\text { ENSDARG000000983 }\end{array}$ & $\begin{array}{l}\text { ENSDART000000009889 } \\
\text { ENSDART000000989 }\end{array}$ & $\begin{array}{l}\text { D.r.25378.1.A1_at,Dr.24858.1.S1_at } \\
\text { D.25378.1.A1 at.D.24858.1.S1 at }\end{array}$ & $\begin{array}{l}\text { zgc:110040 } \\
\text { zgc: } 110540\end{array}$ & 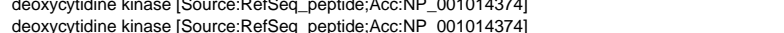 & $\begin{array}{l}-1350 \\
-1580\end{array}$ & $\begin{array}{l}-1325 \\
-1275\end{array}$ & 1 & cgctg \\
\hline ENSDARG00000010312 & ENSDARTO00000010537 & $\begin{array}{l}\text { D..2538.8.1.A1_at,Dr.24858.1.S1_at } \\
\text { Dr.3613.1.S1 at }\end{array}$ & & & -3990 & $\begin{array}{l}-1<5 \\
-3985\end{array}$ & -1 & $\begin{array}{l}\text { agcgeg } \\
\text { tgcotg }\end{array}$ \\
\hline ENSARG00000010312 & ENSDART00000010537 & 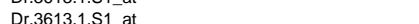 & & & -1870 & -1065 & -1 & gacta \\
\hline ENSDARG00000010312 & ENSDART00000010537 & Dr.3613.1.S1 at & & & -1737 & -1732 & 1 & $\begin{array}{l}\text { goclg } \\
\text { gactg }\end{array}$ \\
\hline ENSDARG00000010312 & ENSDART00000010537 & Dr.3613.1.S1_at & & & -125 & -120 & 1 & tgcgtg \\
\hline ENSDARG00000010420 & ENSDART00000049420 & Dr.8090.1.A1_at & ndrg11 & N-myc downstream regulated gene 1, like [Source:RefSeq_peptide;Acc:NP_956986] & -3038 & -3033 & 1 & agcgtg \\
\hline ENSDARG00000010420 & ENSDART00000049420 & Dr.8090.1.A1_at & ndrg11 & N-myc downstream regulated gene 1, like [Source:RefSeq_peptide;Acc:NP_956986] & -2860 & -2855 & -1 & agctgtg \\
\hline ENSDARG00000010481 & ENSDART00000013575 & Dr.25498.1.S1_at & NP_956002.1 & & -2137 & -2132 & -1 & ggcgtg \\
\hline ENSDARG00000010481 & ENSDART00000013575 & Dr.25498.1.S1_at & NP_956002.1 & basic leucine zipper and W2 domains 1 [Source:RefSeq_peptide;Acc:NP_956002] & -1647 & -1642 & 1 & $\operatorname{tgcgtg}$ \\
\hline ENSDARG00000011312 & ENSDART00000008698 & Dr.24253.1.S1_at & 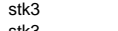 & serine/threonine kinase 3 (STE20 homolog, yeast) [Source:RefSeq_peptide;ACc:NP_-955966] & -3610 & -3605 & -1 & agcgtg \\
\hline $\begin{array}{l}\text { ENSDARG000000011312 } \\
\text { ENSDARG00000011312 }\end{array}$ & 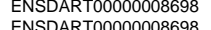 & Dr.24253.1.S1_at & stk3 & serine/threonine kinase 3 (STE20 homolog, yeast) [Source:RefSeq_peptide;AAc:NP_-9555966] & $\begin{array}{l}-3392 \\
-3025\end{array}$ & -3387 & -1 & agcgtg \\
\hline $\begin{array}{l}\text { ENSDARG00000011312 } \\
\text { ENSDARG00000011312 }\end{array}$ & $\begin{array}{l}\text { ENSDART000000008698 } \\
\text { ENSDART0000008698 }\end{array}$ & $\begin{array}{l}\text { DD.242433.1.S1_at } \\
\text { Dr.24531.S1 at }\end{array}$ & $\begin{array}{l}\text { Stt3 } 3 \\
\text { stk3 }\end{array}$ & 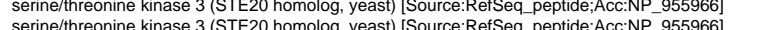 & $\begin{array}{l}-32651 \\
-2651\end{array}$ & $\begin{array}{l}-3020 \\
-2646\end{array}$ & -1 & $\begin{array}{l}\text { agcgtg } \\
\text { agctag }\end{array}$ \\
\hline ENSDARG00000011312 & ENSDART000000008698 & $\begin{array}{l}\text { Di.r.4253.1.S1_at } \\
\text { Dr.24253.S1 at }\end{array}$ & stk3 & 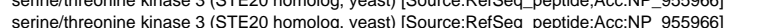 & $\begin{array}{l}-2051 \\
-1477\end{array}$ & $\begin{array}{l}-2440 \\
-1472\end{array}$ & -1 & agcotg \\
\hline ENSARG00000011683 & ENSDART00000013454 & $\begin{array}{l}\text { Dr.8045.1.S1 at } \\
\text { Dr. at }\end{array}$ & NP 955812.2 & HHGP protein [Source:RefSeq peptide:Acc:NP 955812] & 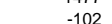 & 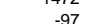 & -1 & 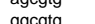 \\
\hline ENSDARG00000012395 & ENSDART00000005436 & Dr.10314.1.S1 a & mmp13 & matrix metalloproteinase 13 [Source:RefSeq_peptide;Acc:NP -958911] & -2663 & -2658 & : & $\begin{array}{l}\text { tgcotg } \\
\text { tcctg }\end{array}$ \\
\hline ENSDARG00000012395 & ENSDART00000005436 & Dr.10314.1.S1 a at & mmp13 & matrix metalloproteinase 13 [Source:RefSeq_peptide;Acc:NP_-958911] & -2528 & -2523 & - & ggcgtg \\
\hline ENSDARG00000012395 & ENSDART00000005436 & Dr.10314.1.51_a at & mmp13 & matrix metalloproteinase 13 [Source:RefSeq- peptide;Acc:NP-958911] & -2276 & -2271 & -1 & ggcgtg \\
\hline ENSDARG00000012395 & ENSDART00000005436 & Dr.10314.1.S1_a_at & mmp13 & matrix metalloproteinase 13 [Source:RefSeq_peptide;Acc:NP_-958911] & -2020 & -2015 & 1 & cgctgtg \\
\hline ENSDARG00000012936 & ENSDART00000012450 & Dr.24920.1.S1_at,Dr.3137.1.A1_at,Dr.5398.1.A1_at & NP_997813.1 & dishevelled 2, dsh homolog [Source:RefSeq_peptide;Acc:NP_-997813] & -3664 & -3659 & 1 & agcgtg \\
\hline ENSDARG00000012936 & ENSDART00000012450 & Dr.24920.1.S1_at,Dr.3137.1.A1_at,Dr.5398.1.A1_at & NP_997813.1 & dishevelled 2, dsh homolog [Source:RefSeq_peptide;Acc:NP_997813] & -4986 & -4981 & 1 & cgcgtg \\
\hline ENSDARG00000012936 & ENSDART00000012450 & Dr.24920.1.S1_at,Dr.3137.1.A1__at,Dr.53988.1.A1_at & NP_-997813.1 & dishevelled 2, dsh homolog [Source:RefSeq_peptide;Acc:NP_997813] & -1028 & -1023 & -1 & agctg \\
\hline ENSDARG00000012936 & ENSDART00000012450 & Dr.24920.1.S1_at,Dr.3137.1.A1_at,Dr.5398.1.A1_at & NP_997813.1 & dishevelled 2, dsh homolog [Source:RefSeq_peptide;Acc:NP_-997813] & -901 & -896 & -1 & ggcgtg \\
\hline $\begin{array}{l}\text { ENSDARG000000012936 } \\
\text { ENSDARG0000012936 }\end{array}$ & $\begin{array}{l}\text { ENSDART000000012450 } \\
\text { ENSPART000001250 }\end{array}$ & 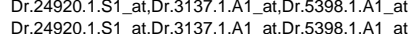 & NP_-99/813.1 & 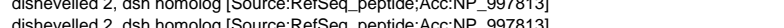 & $\begin{array}{r}-856 \\
-4576\end{array}$ & -8571 & & tgctg \\
\hline ENSDARG00000012936 & ENSDARTO0000012450 & Dr.24920.1.S1_at,Dr.3137.1.A1_at,Dr.5398.1.A1_at & NP_997813.1 & $\begin{array}{l}\text { dishevelled 2, dsh homolog [Source:RefSeq_peptide;Acc:NP-997813] }\end{array}$ & $\begin{array}{l}-476 \\
-748\end{array}$ & $\begin{array}{l}-451 \\
-743\end{array}$ & ${ }_{1}^{-1}$ & cgcgtg \\
\hline ENSARG00000012036 & ENSPART0000001250 & $\begin{array}{ll} & \end{array}$ & NP & 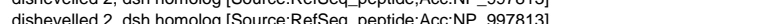 & -664 & -659 & & 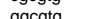 \\
\hline 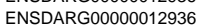 & 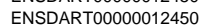 & 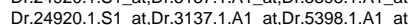 & NP- 997813.1 & 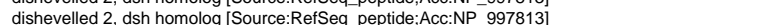 & -276 & -271 & -1 & 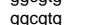 \\
\hline ENSDARG00000013454 & ENSDARTO00000019328 & Dr.16524.1.S1 at & wu:ff12b04 & protein-glutamine gamma-glutamyltransferase [Source:RefSequpeptide;Acc:NP 9978 & -1511 & -1506 & -1 & agcotg \\
\hline ENSDARG00000013454 & ENSDART00000019328 & Dr.16524.1.S1_at & wu:fcc12b04 & protein-glutamine gamma-glutamyltransferase [Source:RefSeqpeptide;Acc:NP_-997821] & -107 & -102 & -1 & ggcgtg \\
\hline
\end{tabular}




\begin{tabular}{|c|c|c|c|c|c|c|c|c|}
\hline Supplementary Table 2 ( & ti.). Location of putative $A$ & & & & & End & Strand & \\
\hline$\frac{\text { Gene ID }}{\text { ENSDARG00000013481 }}$ & $\begin{array}{l}\text { Transcript ID } \\
\end{array}$ & 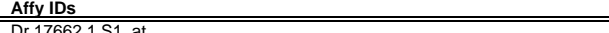 & Symbol & $\begin{array}{l}\text { Description } \\
\end{array}$ & Start $\quad$ it & End & Strand & $\begin{array}{l}\text { Sequence } \\
\text { tacta }\end{array}$ \\
\hline ENSDARG00000013613 & ENSDARTO0000018408 & $\begin{array}{l}\text { D. } \\
\text { Dr.16504.1.A1_at at }\end{array}$ & zgc:112421 & $\begin{array}{l}\text { hypothetical protein LOC554118 [Source:RefSeq_peptide;Acc:NP_OC } \\
\text { hys }\end{array}$ & $\begin{array}{l}-1875 \\
-1875\end{array}$ & -1870 & -1 & 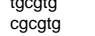 \\
\hline 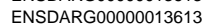 & 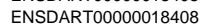 & Dr.16504.1.A1 at & zac:112421 & 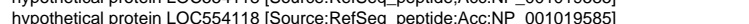 & -1475 & -1470 & -1 & $\begin{array}{l}\text { tgcatg } \\
\text { tgctg }\end{array}$ \\
\hline ENSDARG00000013613 & ENSDART00000018408 & Dr.16504.1.A1_at at & zgc:112421 & 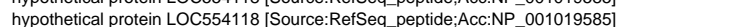 & -1422 & -1417 & -1 & agcgtg \\
\hline & & Dr.1605. S1 at & & serine (or cysteine) proteinase inhibitor, clade A (alpha-1 antiproteinase, antitryp & & & & \\
\hline ENSDARG00000013646 & ENSDART00000019664 & & serpina1 & [Source:RefSeq_peptide;Acc:NP_001013277] & -4808 & -4803 & -1 & agcgtg \\
\hline ENSDARG00000014209 & ENSDART00000023208 & Dr.15930.1.A1_at & NP_001017836.1 & hypothetical protein LOC550534 [Source:RefSeq_peptide;Acc:NP_001017836] & -3248 & -3243 & 1 & agcgtg \\
\hline ENSDARG00000014209 & ENSDART00000023208 & Dr.15930.1.A1_at & $\mathrm{NP}_{-}^{-} 001017836.1$ & hypothetical protein LOC550534 [Source:RefSeq_peptide;Acc:NP_001017836] & -2763 & -2758 & 1 & agcgtg \\
\hline ENSDARG00000014209 & ENSDART00000023208 & Dr.15930.1.A1_at & NP_001017836.1 & hypothetical protein LOC550534 [Source:RefSeq_peptide;Acc:NP_001017836] & -4258 & -4253 & -1 & ggcgtg \\
\hline ENSDARG00000014429 & ENSDART00000017280 & $\begin{array}{l}\text { Dr.4907.1.S1_at } \\
\text { Dr.921.1.A1 at.DrAffx.2.64.S1 at.Dr.921.1.A1 at.DrAffx.2.64.S1 at,Dr.921.1. }\end{array}$ & & fibrinogen gamma polypeptide [Source:RefSeq_peptide;Acc:NP_998219] & -4836 & -4831 & 1 & cgcgtg \\
\hline ENSDARG00000014629 & ENSDART00000020883 & A1_at,DrAffx.2.64.S1_at at & & & -4912 & -4907 & -1 & tgcgtg \\
\hline ENSDARG00000014629 & ENSDART00000020883 & $\begin{array}{l}\text { Dr.921.1.A1_at,DrAffx.2.64.S1_at,Dr.9211.1.A1_at,DrAffx.2.64.S1_at,Dr.921.1. } \\
\text { A1 at.DAAff.2.64.S1 at }\end{array}$ & & & -4071 & -4066 & 1 & agcatg \\
\hline & & $\begin{array}{l}\text { A1 at,DrAAfx.2.644.S1 at } \\
\text { Dr.921.1.A1 at,DrAfx 2.64.S1 at,Dr.921.1.A1 at,DrAffx.2.64.S1 at,Dr.921.1. }\end{array}$ & & & $-40 / 1$ & & -1 & agcgtg \\
\hline ENSDARG00000014629 & ENSDART00000049779 & A1_at,DrAfffx.2.64.S1_at at & & & -4912 & -4907 & -1 & tgcgtg \\
\hline ENSDARG00000014629 & ENSDART00000049779 & $\begin{array}{l}\text { Dr.921.1.A1_at,irrAAxx.2.64.S1_at,Dr.921.1.A1__at,DrAftx.2.64.S1_at,Dr.921.1. } \\
\text { A1_at,DrAffx.2.64.S1_at }\end{array}$ & & & -4071 & -4066 & -1 & agcgtg \\
\hline & & Dr.921.1.A1_at,Draffx.2.64.S1_at,Dr.921.1.A1_at,DrAffx.2.64.S1_at,Dr.921.1. & & & & & & \\
\hline ENSDARG00000014629 & ENSDART00000066174 & A1_at,DrAffx.2.64.S1_at & & & -4912 & -4907 & -1 & tgcgtg \\
\hline ENSDARG00000014629 & ENSDART00000066174 & $\begin{array}{l}\text { Dr.921.1.A1_at,DrAffx.2.64.S1_at,Dr.921.1.A1_at,DrAffx.2.64.S1_at,Dr.921.1. } \\
\text { A1 atDreffx 264.S1 at. }\end{array}$ & & & & & -1 & \\
\hline ENSDARG00000014947 & ENSDART00000026576 & 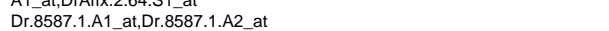 & igftpp1 & insulin-like growth factor binding protein 1 [Source:RefSeq_peptide;Acc:NP_775390] & -3978 & -3973 & -1 & 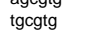 \\
\hline ENSDARG00000014947 & ENSDART00000026576 & Dr.8587.1.A1_at,Dr.8587.1.A2_at & igfbp1 & insulin-like growth factor binding protein 1 [Source:RefSeq_peptide;Acc:NP-775390] & -3265 & -3260 & -1 & tgcgtg \\
\hline ENSDARG00000014947 & ENSDART00000026576 & Dr.8587.1.A1_at,Dr.8587.1.A2_at & igfbp1 & insulin-like growth factor binding protein 1 [Source:RefSeq_peptide;Acc:NP_-775390] & -2732 & -2727 & -1 & tgcgtg \\
\hline ENSDARG00000014947 & ENSDART00000026576 & Dr.8587.1.A1_at,Dr.8587.1.A2_at & igfbp1 & insulin-like growth factor binding protein 1 [Source:RefSeq_peptide;Acc:NP_-775390] & -1510 & -1505 & 1 & cgcgtg \\
\hline ENSDARG00000014947 & ENSDART00000026576 & Dr.8587.1.A1_at,Dr.8587.1.A2_at & igfbp1 & insulin-like growth factor binding protein 1 [Source:RefSeq_peptide;Acc:NP_-775390] & -1468 & -1463 & -1 & cgcgtg \\
\hline ENSDARG00000014947 & ENSDART00000026576 & Dr.8587.1.A1_at,Dr.8587.1.A2_at & igfbp1 & insulin-like growth factor binding protein 1 [Source:RefSeq_peptide;Acc:NP_775390] & -1419 & -1414 & -1 & cgcgtg \\
\hline ENSDARG00000014947 & $\begin{array}{l}\text { ENSDART000000026576 } \\
\text { ENSTR }\end{array}$ & Dr.8587.1.A1_at,Dr.8587.1.A2_at & $\begin{array}{l}\text { igftbp1 } \\
\text { iofthn1 }\end{array}$ & insulin-like growth factor binding protein 1 [Source:RefSeq_peptide;Acc:NP-775390] & -1375 & -1370 & 1 & $\operatorname{tgcgtg}$ \\
\hline $\begin{array}{l}\text { ENSDARG0000000149947 } \\
\text { ENSDARG0000014947 }\end{array}$ & $\begin{array}{l}\text { ENSDART000000026576 } \\
\text { ENDART0000026576 }\end{array}$ & 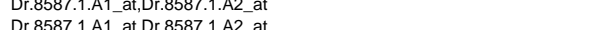 & $\begin{array}{l}\text { igfbpl } 1 \\
\text { igftbp1 }\end{array}$ & 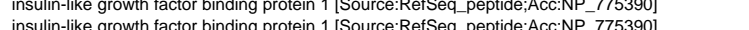 & $\begin{array}{l}-1229 \\
-1076\end{array}$ & $\begin{array}{l}-1224 \\
-1071\end{array}$ & 1 & cgcgtg \\
\hline $\begin{array}{l}\text { ENSARG00000014944 } \\
\text { ENSDARG0000014947 }\end{array}$ & $\begin{array}{l}\text { ENSARI000000265 } \\
\text { ENSDART0000026576 }\end{array}$ & 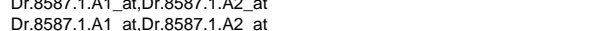 & 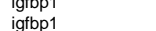 & 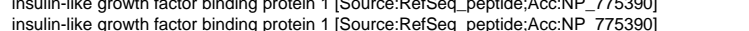 & $\begin{array}{l}-1229 \\
-229\end{array}$ & -224 & -1 & $\begin{array}{l}\text { cgcggtg } \\
\text { agcgtg }\end{array}$ \\
\hline ENSDARG00000014907 & FNSDATOR0000202676 & 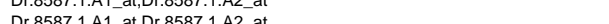 & 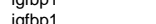 & 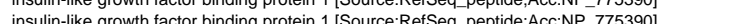 & 年 & (-224 & -1 & 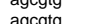 \\
\hline ENSDARG00000014947 & ENSDART00000026576 & Dr.8587.1.A1 at.Dr.8587.1.A2 at & igfbp1 & insulin-like growth factor binding protein 1 [Source:RefSeg peptide:Acc:NP 775390 ] & 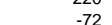 & 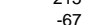 & 1 & acta \\
\hline ENSDARG00000015866 & ENSDART00000019110 & Dr.728.4.S1_at,Dr.728.4.S1_at,Dr.728.4.S1_at & & & -3239 & -3234 & -1 & tgcotg \\
\hline ENSDARG00000015866 & ENSDART00000019110 & Dr.728.4.S1_at,Dr.728.4.S1_at,Dr.728.4.S1_at & & & -401 & -396 & 1 & cgcgtg \\
\hline ENSDARG00000015866 & ENSDART00000058963 & Dr.728.4.S1_at,Dr.728.4.S1__at,Dr.728.4.S1_at & & & -3537 & -3532 & -1 & tgcgtg \\
\hline ENSDARG00000015866 & ENSDART00000058963 & Dr.728.4.S1_at,Dr.728.4.S1_at,Dr.728.4.S1_at & & & -699 & -694 & 1 & cgctgtg \\
\hline ENSDARG00000015866 & ENSDART00000058964 & Dr.728.4.S1_at,Dr.728.4.S1_at,Dr.728.4.S1_at & & & -3239 & -3234 & -1 & tgcgtg \\
\hline ENSDARG00000015866 & ENSDART00000058964 & Dr.728.4.S1_at,Dr.728.4.S1_at,Dr.728.4.S1_at & & & -401 & -396 & 1 & cgcgtg \\
\hline $\begin{array}{l}\text { ENSDARG000000016059 } \\
\text { ENSTA }\end{array}$ & $\begin{array}{l}\text { ENSDART000000021065 } \\
\text { ENSTART00002105 }\end{array}$ & $\begin{array}{ll}\text { Dr.13182.1.Ā1_at } & -\bar{A} \\
\end{array}$ & rab5b & RAB5B, member RAS oncogene family [Source:RetSeq_peptide;Acc:NP_998050] & -3042 & -3037 & -1 & tgcgtg \\
\hline $\begin{array}{l}\text { ENSDARG000000016059 } \\
\text { ENDARG }-3000016059\end{array}$ & $\begin{array}{l}\text { ENDARART000000021065 } \\
\text { ENSPART00000021065 }\end{array}$ & 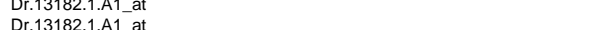 & $\begin{array}{llll}r a b 5 b \\
r a b 5 b\end{array}$ & 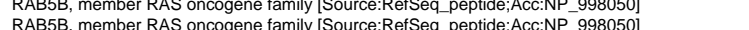 & $\begin{array}{l}-2041 \\
-672\end{array}$ & $\begin{array}{l}-2036 \\
-667\end{array}$ & 1 & agcgtt \\
\hline $\begin{array}{l}\text { ENSARGOU0000016059 } \\
\text { ENSDARG0000016059 }\end{array}$ & $\begin{array}{l}\text { ENSARI000000211065 } \\
\text { ENSDART00000021065 }\end{array}$ & $\begin{array}{l}\text { DD.r.31828.1.A.A1_at } \\
\text { Dr.13182. A1 at }\end{array}$ & $\begin{array}{l}\text { rabsb } \\
\text { rab5b }\end{array}$ & 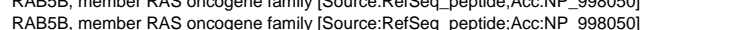 & -6468 & -6663 & 1 & $\begin{array}{l}\text { agcgtg } \\
\text { agctag }\end{array}$ \\
\hline ENSDARG00000016448 & ENSDART00000014979 & $\begin{array}{lll} & \end{array}$ & va3 & Vitellogenin 3 (Fragment) [Source'Uniprot/SPTREMBL-Acc-OOgDFTg] & -1530 & $\begin{array}{l}-4525 \\
-4525\end{array}$ & -1 & 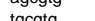 \\
\hline ENSDARG00000016771 & ENSDART00000003845 & Dr.1889.2.A1 a at,Dr.1889.1.S1 at & tta & transferrin-a [Source:RefSeq peptide;Acc:NP 001015057] & 年3533 & 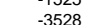 & 1 & $\begin{array}{l}\text { tocta } \\
\text { tocta }\end{array}$ \\
\hline ENSDARG00000016771 & ENSDART00000003845 & Dr.1889.2.A1 a at,Dr.1889.1.S1 at & tfa & transferrin-a [Source:RefSeq peptide;Acc:NP 001015057$]$ & -3351 & -3346 & 1 & agcotg \\
\hline ENSDARG00000016771 & ENSDART00000003845 & Dr.1889.2.A1_a_at,Dr.1889.1.S1_at at & tfa & transferrin-a [Source:RefSeq_peptide;Acc:NP_001015057] & -2761 & -2756 & -1 & cgcgtg \\
\hline ENSDARG00000016771 & ENSDART00000003845 & Dr.1889.2.A1_a_at,Dr.1889.1.S1_at & tfa & transferrin-a [Source:RefSeq_peptide;Acc:NP_001015057] & -2755 & -2750 & -1 & tgcgtg \\
\hline ENSDARG00000016771 & ENSDART00000003845 & Dr.1889.2.A1_a_at,Dr.1889.1.S1_at & tfa & transferrin-a [Source:RefSeq_peptide;Acc:NP_001015057] & -4965 & -4960 & -1 & tgcgtg \\
\hline ENSDARG00000016771 & ENSDART00000003845 & Dr.1889.2.A1_a_at,Dr.1889.1.S1_at & tfa & transferrin-a [Source:RefSeq_peptide;Acc:NP_001015057] & -3533 & -3528 & 1 & tgcgtg \\
\hline ENSDARG00000016771 & ENSDART00000003845 & Dr.1889.2.A1_a_at,Dr.1889.1.S1_at & $\mathrm{tfa}$ & transferrin-a [Source:RefSeq_peptide;Acc:NP_001015057] & -3351 & -3346 & 1 & agcgtg \\
\hline ENSDARG00000016771 & ENSDART00000003845 & Dr.1889.2.A1_a_at,Dr.1889.1.S1_at & tfa & transferrin-a [Source:RefSeq_peptide;Acc:NP_001015057] & -2761 & -2756 & -1 & cgcgtg \\
\hline $\begin{array}{l}\text { ENSDARG0000000016771 } \\
\text { ENDARG }\end{array}$ & $\begin{array}{l}\text { ENSDART000000003885 } \\
\text { ENDART000000385 }\end{array}$ & $\begin{array}{l}\text { Dr.1889.2.A1_a_at,Dr.1889.1.S1_at } \\
\text { D.18892 A1 a a at } 18891 \text { S1 at }\end{array}$ & $\begin{array}{l}\text { tfa } \\
\text { ta }\end{array}$ & 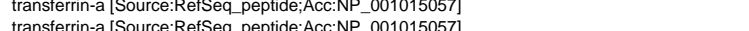 & -2755 & $\begin{array}{c}-2750 \\
-9060\end{array}$ & -1 & tgctg \\
\hline $\begin{array}{l}\text { ENSARGO00000016/1 } \\
\text { ENSDARG0000016825 }\end{array}$ & $\begin{array}{l}\text { ENSARI } \\
\text { ENSDART00000000302355 }\end{array}$ & 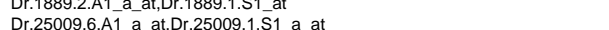 & NP 739573.1 & 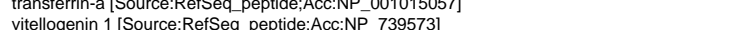 & $\begin{array}{l}-4365 \\
-3552\end{array}$ & $\begin{array}{l}-4560 \\
-3547\end{array}$ & -1 & 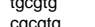 \\
\hline ENSDAGG000001625 & ENSDATOROP0050 & $\begin{array}{lll} & -1\end{array}$ & 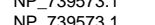 & 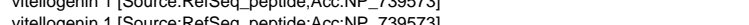 & 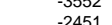 & 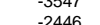 & 1 & 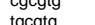 \\
\hline ENSDARG00000016825 & ENSDART00000050238 & Dr.25009.6.A1 a at.Dr.25009.1.S1 a at at & NP 739573.1 & 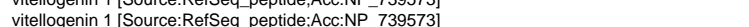 & 醇 & 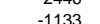 & -1 & 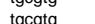 \\
\hline ENSDARG00000016825 & ENSDART00000050238 & Dr.25009.6.A1 a at,Dr.25009.1.S1 a at & NP 739573.1 & 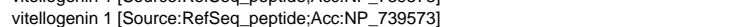 & -3552 & -3547 & 1 & 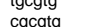 \\
\hline ENSDARG00000016825 & ENSDART00000050238 & Dr.25009.6.A1_a_at,Dr.25009.1.S1_a_at & $\mathrm{NP}_{-}^{-} 739573.1$ & 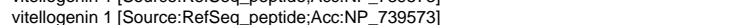 & -2451 & -2446 & -1 & tgcgtg \\
\hline ENSDARG00000016825 & ENSDART00000050238 & Dr.25009.6.A1_a_at,Dr.25009.1.S1_a__at & NP_-739573.1 & vitellogenin 1 [Source:RefSeq_peptide;Acc:NP_739573] & -1138 & -1133 & -1 & tgcgtg \\
\hline ENSDARG00000017619 & ENSDART00000026842 & Dr.25379.1.S1_at,Dr.3025.3.S1_at,Dr.25379.1.S1_at,Dr.3025.3.S1_at & & & -3793 & -3788 & 1 & tgcgtg \\
\hline ENSDARG00000017619 & ENSDART00000026842 & Dr.25379.1.S1_at,Dr.3025.3.S1_at,Dr.25379.1.S1_at,Dr.3025.3.S1_at & & & -3785 & -3780 & 1 & tgcgtg \\
\hline ENSDARG00000017619 & ENSDART00000026842 & Dr.25379.1.S1_at,Dr.3025.3.S1_at,Dr.25379.1.S1_at,Dr.3025.3.S1_at & & & -3587 & -3582 & -1 & tgcgtg \\
\hline $\begin{array}{r}\text { ENSDARG000000017619 } \\
\text { EN }\end{array}$ & ENSDART00000026842 & Dr.25379.1.S1_at,Dr.3025.3.S1_at,Dr.25379.1.S1__at,Dr.3025.3.S1_at & & & -3364 & -3359 & 1 & ggcgtg \\
\hline ENSDARG00000017619 & ENSDART00000026842 & 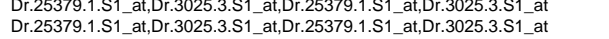 & & & $\begin{array}{l}-2980 \\
-2502\end{array}$ & $\begin{array}{l}-2975 \\
-2497\end{array}$ & $\begin{array}{l}1 \\
1\end{array}$ & $\begin{array}{l}\text { agctg } \\
\text { tgcctg }\end{array}$ \\
\hline ENSDARG00000017619 & ENSDART00000026842 & Dr.25379.1.S1_at,Dr.3025.3.S1_at,Dr.25379.1.S1_at,Dr.3025.3.S1_at & & & -1174 & -1169 & -1 & tgcgtg \\
\hline ENSDARG00000017619 & ENSDART00000038499 & & & & -2596 & -2591 & & $\begin{array}{l}\text { tgcgtg } \\
\text { tgctg }\end{array}$ \\
\hline 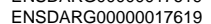 & 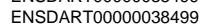 & 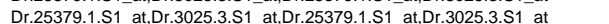 & & & -24243 & - 4238 & -1 & tacato \\
\hline ENSDARG00000017619 & 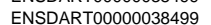 & 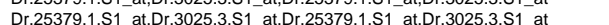 & & & -4339 & -4234 & -1 & $\begin{array}{l}\text { tgcatg } \\
\text { tacta }\end{array}$ \\
\hline ENSDARG00000017619 & ENSDARTO00000038499 & 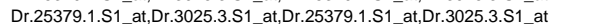 & & & -4053 & -4048 & -1 & tgcgtg \\
\hline ENSDARG00000017619 & ENSDART00000026842 & Dr.25379.1.S1_at,Dr.3025.3.S1_at,Dr.25379.1.S1_at,Dr.3025.3.S1_at & & & -3793 & -3788 & 1 & tgcgtg \\
\hline ENSDARG00000017619 & ENSDART00000026842 & Dr.25379.1.S1_at,Dr.3025.3.S1_at,Dr.25379.1.S1_at,Dr.3025.3.S1__at & & & -3785 & -3780 & 1 & tgcgtg \\
\hline ENSDARG00000017619 & ENSDART00000026842 & Dr.25379.1.S1_at,Dr.3025.3.S1_at,Dr.25379.1.S1_at,Dr.3025.3.S1_at & & & -3587 & -3582 & -1 & $\operatorname{tgcgtg}$ \\
\hline ENSDARG00000017619 & ENSDART00000026842 & Dr.25379.1.S1_at,Dr.3025.3.S1_at,Dr.25379.1.S1_at,Dr.3025.3.S1_at & & & -3364 & -3359 & 1 & ggcgtg \\
\hline ENSDARG00000017619 & ENSDART00000026842 & Dr.25379.1.S1_at,Dr.3025.3.S1_at,Dr.25379.1.S1_at,Dr.3025.3.S1_at & & & -2980 & -2975 & 1 & agcgtg \\
\hline $\begin{array}{l}\text { ENSDARG000000017619 } \\
\text { ENSDARG0000017619 }\end{array}$ & $\begin{array}{l}\text { ENSDART0000000262682 } \\
\text { ENSDART0000026842 }\end{array}$ & 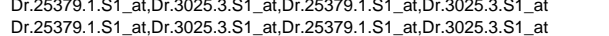 & & & $\begin{array}{l}-2502 \\
-1174\end{array}$ & $\begin{array}{l}-2497 \\
-1169\end{array}$ & $\begin{array}{c}1 \\
-1\end{array}$ & $\begin{array}{l}\text { tggctg } \\
\text { tgcgtg }\end{array}$ \\
\hline $\begin{array}{l}\text { ENSDARG00000001/1619 } \\
\text { ENSDARG0000017619 }\end{array}$ & ENSDARTO0000038499 & & & & -2596 & -2591 & 1 & tgcatg \\
\hline 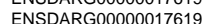 & ENSDARTO0000038499 & Dr.25379.1.S1 at.Dr.3025.3.S1 at.Dr.2537 & & & & 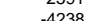 & 1 & toclat \\
\hline ENSPRAG00000017669 & ENSPART00000338999 & & & & $\begin{array}{l}-439 \\
-439\end{array}$ & 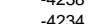 & -1 & 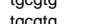 \\
\hline ENSDARG00000017619 & 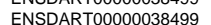 & 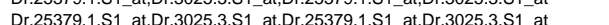 & & & -4053 & -4048 & -1 & tacata \\
\hline ENSDARG00000017624 & ENSDART00000012644 & 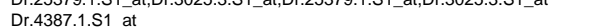 & krt4 & fSeq peptide:Acc:NP 571584] & -1874 & -1869 & 1 & tgcgtg \\
\hline ENSDARG00000017624 & ENSDART00000012644 & Dr.4387.1.S1_at & ktt 4 & keratin 4 [Source:RefSeq_peptide;Acc:NP_571584] & -1435 & -1430 & -1 & tgcgtg \\
\hline ENSDARG00000017624 & ENSDART00000012644 & Dr.4387.1.S1_at & krt4 & keratin 4 [Source:RefSeq_peptide;Acc:NP_571584] & -9 & -4 & -1 & tgcgtg \\
\hline ENSDARG00000018404 & ENSDART00000020750 & Dr.1372.1.S1_at & krt18 & keratin 18 [Source:RefSeq_peptide;Acc:NP__848524] & -2408 & -2403 & 1 & ggcgtg \\
\hline ENSDARG00000018404 & ENSDART00000020750 & Dr.1372.1.S1_at & kr18 & keratin 18 [Source:RefSeq_peptide;Acc:NP_848524] & -4641 & -4636 & -1 & cgcgtg \\
\hline $\begin{array}{l}\text { ENSDARG000000018404 } \\
\text { ENSARG00000018529 }\end{array}$ & $\begin{array}{l}\text { ENSDART00000020750 } \\
\text { ENDART0000020541 }\end{array}$ & $\begin{array}{l}\text { Dr.1372.1.S1_at } \\
\text { Dr. } 42491 \mathrm{~S} 1 \text { at }\end{array}$ & $\begin{array}{l}\text { krt18 } \\
\text { liff }\end{array}$ & 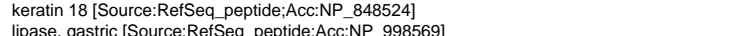 & $\begin{array}{r}-37 \\
-1538\end{array}$ & $\begin{array}{r}-32 \\
-1533\end{array}$ & 1 & $\begin{array}{l}\text { tgcgtg } \\
\text { cocata }\end{array}$ \\
\hline & $\begin{array}{l}\text { ENSDART0000000200451 } \\
\text { ENSDART0000020541 }\end{array}$ & $\begin{array}{l}\text { Dr.4249.1.S1_at } \\
\text { Dr.4249.1.S1 at }\end{array}$ & $\begin{array}{l}\text { lipt } \\
\text { lipf }\end{array}$ & 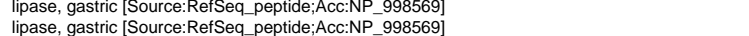 & $\begin{array}{l}-1538 \\
-1050\end{array}$ & $\begin{array}{l}-1533 \\
-1045\end{array}$ & $\begin{array}{l}1 \\
1\end{array}$ & $\begin{array}{l}\text { cgcgtg } \\
\text { gacgta }\end{array}$ \\
\hline ENSDARG00000018529 & ENSDART00000020541 & Dr.4249.1.S1_at & lipf & 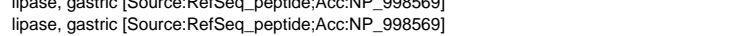 & ${ }_{-858}^{-1050}$ & $\begin{array}{l}-8045 \\
-853\end{array}$ & 1 & $\begin{array}{l}\text { ggcgge } \\
\text { agcotg }\end{array}$ \\
\hline
\end{tabular}




\begin{tabular}{|c|c|c|c|c|c|c|c|c|}
\hline $\begin{array}{l}\text { Supplementary Table } 2 \text { ( } \\
\text { Gene ID }\end{array}$ & 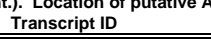 & Affy IDs & Symbol & Description & Start & End & Strand & Sequence \\
\hline 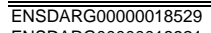 & ENSDART00000020541 & Dr.4249.1.S1_at & & lipase, gastric [Source:RefSeq_peptide;ACc:NP_998569] & 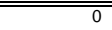 & 25 & $2-1$ & 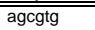 \\
\hline ENSDARG00000018921 & ENSDART00000023006 & Dr.9450.1.A1_at & NP_956912.1 & hypothetical protein LOC393590 [Source:RefSeq_peptide;Acc:NP_956912] & -1589 & -1584 & 1 & tgcgtg \\
\hline ENSDARG00000018921 & ENSDART00000023006 & Dr.9450.1.A1_at & NP_956912.1 & hypothetical protein LOC393590 [Source:RefSeq_peptide;Acc:NP_956912] & -1563 & -1558 & 1 & tgcgtg \\
\hline ENSDARG000000018921 & ENSDART00000023006 & Dr.9450.1.A1_at & NP_956912.1 & hypothetical protein LOC393590 [Source:RefSeq_peptide;Acc:NP_956912] & -1559 & -1554 & 1 & $\operatorname{tgctg}$ \\
\hline ENSDARG00000018921 & ENSDART00000023006 & 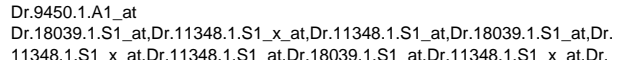 & NP_956912.1 & hypothetical protein LOC393590 [Source:RefSeq_peptide;ACc:NP_956912] & 25 & 30 & -1 & cgcgtg \\
\hline ENSDARG00000019057 & ENSDART00000003224 & 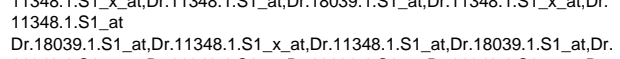 & & & -3377 & -3372 & -1 & $\operatorname{tgctg}$ \\
\hline ENSDARG00000019057 & ENSDART00000048630 & 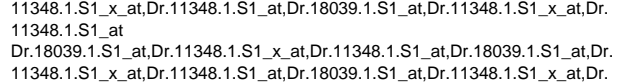 & & & -3377 & -3372 & -1 & $\operatorname{tgcgtg}$ \\
\hline ENSDARG00000019057 & ENSDART00000050015 & 11348.1.S1_at & & & -3377 & -3372 & -1 & tgcgtg \\
\hline ENSDARG00000019302 & ENSDART00000007649 & Dr.25285.1.S1_at,Dr.11211.1.S1_at & zgc:73223 & hypothetical protein MGC73223 [Source:RefSeq_peptide;Acc:NP_957126] & -3810 & -3805 & 1 & agcgtg \\
\hline ENSDARG00000019302 & ENSDART00000007649 & Dr.25285.1.S1_at,Dr.11211.1.S1_at & zgc:73223 & hypothetical protein MGC73223 [Source:RefSeq_peptide;Acc:NP_957126] & -2001 & -1996 & 1 & $\operatorname{tgcgtg}$ \\
\hline & $\begin{array}{l}\text { ENSDART000000007649 } \\
\text { ENSART000000749 }\end{array}$ & Dr.25285.1.S1_at,Dr.11211.1.S1_at & zgc:73223 & hypothetical protein MGC73223 [Source:RefSeq_peptide;Acc:NP_957126] & -348 & -343 & -1 & $\operatorname{tgcgtg}$ \\
\hline $\begin{array}{l}\text { ENSDDRG000000019302 } \\
\text { ENSDARG0000019469 }\end{array}$ & $\begin{array}{l}\text { ENSDART000000007649 } \\
\text { ENDART0000011211 }\end{array}$ & $\begin{array}{l}\text { Dr.25285.1.S1_at,Dr.11211.1.S1_at } \\
\text { Dr242531S1 at }\end{array}$ & zgc:73223 & 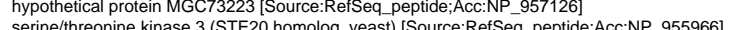 & -4447 & -4442 & 1 & tgcgtg \\
\hline & $\begin{array}{l}\text { ENSDART0000000111211 } \\
\text { ENSDART000001211 }\end{array}$ & $\begin{array}{l}\text { DD.2424233.1.S1__at } \\
\text { Dr.24253.S1_at }\end{array}$ & $\begin{array}{l}\text { SktK3 } \\
\text { stk3 }\end{array}$ & 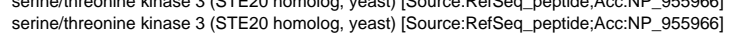 & -2701 & $\begin{array}{l}-3598 \\
-2696\end{array}$ & $\begin{array}{l}-1 \\
-1\end{array}$ & agctg \\
\hline ENSDARG000000019469 & ENSDARTO00000011211 & Dr.24253.1.S1_at at & stk3 & $\begin{array}{l}\text { serine/threonine kinase } 3 \text { (STE20 homolog, yeast) [Source:RefSeq_peptide;Acc:NP_955966] } \\
\text { (ST) }\end{array}$ & -4704 & -4699 & -1 & agcgtg \\
\hline ENSDARG00000019469 & ENSDART00000011211 & Dr.24253.1.S1_at & stk3 & serine/threonine kinase 3 (STE20 homolog, yeast) [Source:RefSeq_peptide;ACC:NP_955966] & -1423 & -1418 & -1 & agcgtg \\
\hline $\begin{array}{l}\text { ENSDARG000000019466 } \\
\text { ENDARG00000019469 }\end{array}$ & ENSDART00000011211 & Dr.24253.1.S1_at & stk3 & serine/threonine kinase 3 (STE20 homolog, yeast) [Source:RefSeq_peptide;Acc:NP_955966] & -1407 & -1402 & 1 & ggcgtg \\
\hline $\begin{array}{l}\text { ENSDDRG000000019649 } \\
\text { ENSDARG0000019469 }\end{array}$ & $\begin{array}{l}\text { ENSDART0000000112111 } \\
\text { ENDDART0000011211 }\end{array}$ & $\begin{array}{l}\text { Dr.24253.1.S1_at } \\
\text { DD.242531. S1 at }\end{array}$ & stk3 & serine/threonine kinase 3 (STE20 homolog, yeast) [Source:RefSeq_peptide;Acc:NP_955966] & -1232 & -1227 & -1 & tgcgtg \\
\hline ENSDARG00000019469 & $\begin{array}{l}\text { ENSDART0000000111211 } \\
\text { ENSDART000001211 }\end{array}$ & $\begin{array}{l}\text { DD.2424233.1.S1__at } \\
\text { Dr.24253.1.S1_at }\end{array}$ & 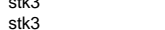 & 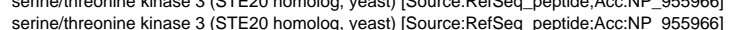 & $\begin{array}{l}-1189 \\
-1139\end{array}$ & $\begin{array}{l}-11134 \\
-134\end{array}$ & $\begin{array}{l}-1 \\
-1\end{array}$ & tgcgitio \\
\hline ENSDARG00000019469 & ENSDART00000011211 & Dr.24253.1.S1_at & stk3 & serine/threonine kinase 3 (STE20 homolog, yeast) [Source:RefSeq_peptide;ACC:NP_955966] & -1084 & -1079 & -1 & cgcgtg \\
\hline ENSDARG00000019469 & ENSDART00000011211 & Dr.24253.1.S1_at & stk3 & serine/threonine kinase 3 (STE20 homolog, yeast) [Source:RefSeq_peptide;ACC:NP_955966] & -1056 & -1051 & -1 & cgcgtg \\
\hline 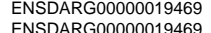 & ENSDART00000011211 & Dr.24253.1.S1_at & stk3 & serine/threonine kinase 3 (STE20 homolog, yeast) [Source:RefSeq_peptide;Acc:NP_955966] & -1016 & -1011 & -1 & cgcgtg \\
\hline $\begin{array}{l}\text { ENSDARG000000019469 } \\
\text { ENSDARG000001969 }\end{array}$ & $\begin{array}{l}\text { ENSDART000000011211 } \\
\text { ENDART0000011211 }\end{array}$ & $\begin{array}{l}\text { Dr.24253.1.S1_at } \\
\text { DP.24531. S1 at }\end{array}$ & stk3 & serine/threonine kinase 3 (STE20 homolog, yeast) [Source:RefSeq_peptide;Acc:NP_955966] & -623 & -618 & 1 & cgcgtg \\
\hline ENSDARG00000019469 & ENSDART00000011211 & $\begin{array}{l}\text { DD.242533.1.S1_at } \\
\text { Dr.24253.1.S1_at }\end{array}$ & $\begin{array}{l}\text { Stk33 } \\
\text { stk3 }\end{array}$ & 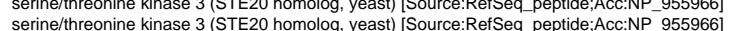 & $\begin{aligned}-159 & -4337\end{aligned}$ & $\begin{array}{l}-1542 \\
-432\end{array}$ & -1 & tgcgtg \\
\hline ENSDARG00000019469 & ENSDART00000011211 & $\begin{array}{l}\text { Dr.24253.1.S1_at } \\
\text { Dr. at }\end{array}$ & stk3 & serine/threonine kinase 3 (STE20 homolog, yeast) [Source:RefSeq_peptide;ACC:NP_955966] & -4922 & -4917 & -1 & 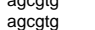 \\
\hline ENSDARG00000019862 & ENSDART00000009054 & Dr.913.1.S1 at at & zgc:63909 & 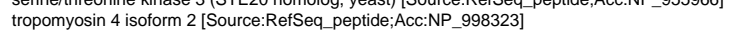 & -3027 & -3022 & 1 & 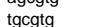 \\
\hline ENSDARG00000019862 & ENSDART00000009054 & Dr.913.1.S1_at & zgc:63909 & tropomyosin 4 isoform 2 [Source:RefSeq peptide;Acc:NP_998323] & -2322 & -2317 & 1 & agcgtg \\
\hline ENSDARG00000019862 & ENSDART00000009054 & Dr.913.1.S1_at & zgc:63909 & tropomyosin 4 isoform 2 [Source:RefSeq__eptide;Acc:NP_998323] & -1865 & -1860 & 1 & agcotg \\
\hline ENSDARG00000020007 & ENSDART00000042990 & Dr.1377.1.A1_at,Dr.23532.1.A1_at,Dr.5521.1.S1_at & & collagen, type I, alpha 2 [Source:RefSeq_peptide;Acc:NP_892013] & -3691 & -3686 & -1 & cgcgtg \\
\hline ENSDARG00000020007 & ENSDART00000042990 & Dr.1377.1.A1_at,Dr.23532.1.A1_at,Dr.5521.1.S1_at & col1a2 & collagen, type I, alpha 2 [Source:RefSeq_peptide;Acc:NP_892013] & -2964 & -2959 & 1 & cgcgtg \\
\hline ENSDARG00000020007 & ENSDART00000042990 & Dr.1377.1.A1_at,Dr.23532.1.A1_at,Dr.5521.1.S1_at & col1a2 & collagen, type I, alpha 2 [Source:RefSeq_peptide;Acc:NP_892013] & -2808 & -2803 & 1 & tgcgtg \\
\hline ENSDARG00000020007 & ENSDART00000042990 & Dr.1377.1.A1_at,Dr.23532.1.A1_at,Dr.5521.1.S1_at & colla2 & collagen, type I, alpha 2 [Source:RefSeq_peptide;Acc:NP_892013] & -909 & -904 & -1 & tgcgtg \\
\hline ENSDARG000000020007 & ENSDART00000042990 & Dr.1377.1.A1_at,Dr.23532.1.A1_at,Dr.5521.1.S1_at & colla2 & collagen, type I, alpha 2 [Source:RefSeq_peptide;Acc:NP_892013] & -234 & -229 & -1 & cgcgtg \\
\hline $\begin{array}{l}\text { ENSDDRG000000020007 } \\
\text { ENSDARG0000020190 }\end{array}$ & $\begin{array}{l}\text { ENSDART000000042990 } \\
\text { ENSART0000003437 }\end{array}$ & Dr.1377.1.A1_at,Dr.23532.1.A1_at,Dr.5521.1.S1_at & col1a2 & collagen, type I, alpha 2 [Source:RefSeq_peptide;Acc:NP_892013] & 6 & 11 & -1 & tgcgtg \\
\hline ENSDARG00000020190 & $\begin{array}{l}\text { ENSDART0000000033347 } \\
\text { ENSDART0000003437 }\end{array}$ & $\begin{array}{l}\text { Dr.250866.1.A1 at } \\
\text { Dr.25086.1.AA_at }\end{array}$ & & & $\begin{array}{l}-240 / 7 \\
-4030\end{array}$ & $\begin{array}{l}-2402 \\
-4025\end{array}$ & $\begin{array}{c}-1 \\
1\end{array}$ & agcgtg \\
\hline 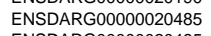 & ENSDART00000011573 & Dr.10314.1.S1_a_at & & & -3871 & -3866 & -1 & tgcgtg \\
\hline ENSDARG00000020485 & ENSDART00000011573 & Dr.10314.1.S1_a_at & & & -3757 & -3752 & 1 & agcgtg \\
\hline ENSDARG00000020485 & ENSDART000000011573 & Dr.10314.1.S1_a_at & & & -4843 & -4838 & -1 & ggcgtg \\
\hline ENSDARG00000020485 & ENSDART00000011573 & Dr.10314.1.S1_a_at & & & -1953 & -1948 & 1 & tgcgtg \\
\hline ENSDARG00000020485 & ENSDART00000011573 & Dr.10314.1.S1_a_at & & & -1091 & -1086 & 1 & ggcgtg \\
\hline ENSDARG00000020893 & ENSDART00000004693 & Dr.17850.1.S1_at & zgc:56592 & zgc:56592 [Source:RefSeq_peptide;Acc:NP_998573] & -1911 & -1906 & 1 & ggctgtg \\
\hline ENSDARG00000020893 & ENSDART00000004693 & Dr.17850.1.S1_at & zgc:56592 & zgc:56592 [Source:RefSeq_peptide;Acc:NP_-998573] & -4527 & -4522 & -1 & cgcgtg \\
\hline ENSDARG00000021443 & ENSDART00000014168 & Dr.24122.1.A1_at & NP_955943.1 & CCCH zinc finger protein C3H-2 [Source:RefSeq_peptide;Acc:NP_955943] & -3319 & -3314 & 1 & cgcgtg \\
\hline ENSDARG00000021443 & ENSDART00000014168 & Dr.24122.1.A1_at & NP_955943.1 & CCCH zinc finger protein C3H-2 [Source:RefSeq_peptide;Acc:NP_955943] & -71 & -66 & 1 & ggcgtg \\
\hline $\begin{array}{l}\text { ENSDARG00000000222295 } \\
\text { ENSDARG0000022295 }\end{array}$ & $\begin{array}{l}\text { ENSDART000000029645 } \\
\text { ENSDART0000029465 }\end{array}$ & $\begin{array}{l}\text { Dr.r.54481.1.S1_at } \\
\text { Dr.15418.1S1 at }\end{array}$ & 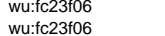 & 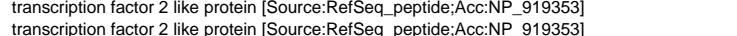 & -4671 & -4666 & 1 & ggcgtg \\
\hline & ENSDART00000029465 & Dr.15418.1.S1_at & wu:fc23306 & 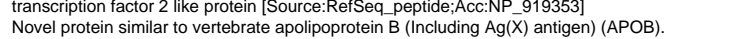 & -152 & -147 & & agcgtg \\
\hline ENSDARG00000022767 & ENSDART00000028709 & Dr.5562.1.S1_at & Q5BLC2_BRARE & [Source:Uniprot/SPTREMBL;Acc:Q5TZ29] & -2584 & -2579 & -1 & $\operatorname{tgcgtg}$ \\
\hline ENSDARG00000022767 & ENSDART00000028709 & Dr.5562.1.S1_at & O5BIC2 BRARE & $\begin{array}{l}\text { Novel protein similar to vertebrate apolipoprotein B (Including Ag( } \mathrm{X} \text { ) antigen) (APOB). } \\
\text {. }\end{array}$ & -780. & -775 & 1 & tacata \\
\hline ENSDARG00000023820 & ENSDART00000038657 & Dr.8390.1.S1 at & zgc:55420 & 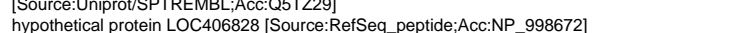 & $\begin{array}{r}-380 \\
-3618\end{array}$ & -3613 & 1 & tagcota \\
\hline ENSDARG00000023820 & ENSDART00000038657 & Dr.8390.1.S1_at & zgc:55420 & $\begin{array}{l}\text { hypothetical protein LOC406828 [Source:RefSeq_peptide;Acc:NP_998672] }\end{array}$ & -3588 & -3583 & -1 & agcgtg \\
\hline ENSDARG00000023820 & ENSDART00000038657 & Dr.8390.1.S1_at & zgc:55420 & hypothetical protein LOC406828 [Source:RefSeq_peptide;ACc:NP_998672] & -2611 & -2606 & -1 & agcgtg \\
\hline ENSDARG00000023820 & ENSDART00000038657 & Dr.8390.1.S1_at & zgc:55420 & hypothetical protein LOC406828 [Source:RefSeq_peptide;Acc:NP_998672] & -2515 & -2510 & -1 & tgcgtg \\
\hline ENSDARG00000023820 & ENSDART00000038657 & Dr.8390.1.S1_at & zgc:55420 & hypothetical protein LOC406828 [Source:RefSeq_peptide;Acc:NP_998672] & -4711 & -4706 & 1 & tgcgtg \\
\hline ENSDARG000000023820 & ENSDART00000038657 & Dr.8390.1.S1_at & zgc:55420 & hypothetical protein LOC406828 [Source:RefSeq_peptide;AAcc:NP_998672] & -1933 & -1928 & 1 & $\operatorname{tgcgtg}$ \\
\hline ENSDARG000000023820 & ENSDART00000038657 & Dr.8390.1.S1_at & zgc:55420 & hypothetical protein LOC406828 [Source:RefSeq_peptide;ACc:NP_998672] & -1521 & -1516 & 1 & cgcgtg \\
\hline $\begin{array}{l}\text { ENSDARG000000003820 } \\
\text { ENDARG0000024017 }\end{array}$ & $\begin{array}{l}\text { ENSDART000000038657 } \\
\text { ENSDART0000033943 }\end{array}$ & $\begin{array}{l}\text { Dr.r390.1.S1.at } \\
\text { DD.16611.A1 at }\end{array}$ & $\begin{array}{l}\text { zgc:55420 } \\
\text { mamd } 1\end{array}$ & 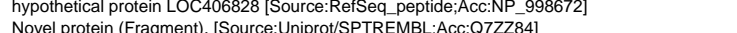 & $\begin{array}{r}-162 \\
-2031\end{array}$ & $\begin{array}{r}-157 \\
-2926\end{array}$ & -1 & tgcgtg \\
\hline $\begin{array}{l}\text { ENSDARG000000024017 } \\
\text { ENSDARG0000024017 }\end{array}$ & $\begin{array}{l}\text { ENSDART000000333943 } \\
\text { ENSDART0000039343 }\end{array}$ & $\begin{array}{l}\text { DD.16681.1.A1_at } \\
\text { Dr.16681.1.A1 at }\end{array}$ & $\begin{array}{l}\text { mamdc1 } \\
\text { mamdc1 }\end{array}$ & 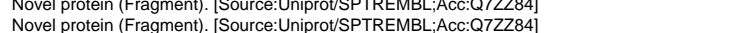 & -2241 & $\begin{array}{l}-2926 \\
-2235\end{array}$ & $\begin{array}{c}1 \\
-1\end{array}$ & $\begin{array}{l}\text { tgcggtg } \\
\text { tocatg }\end{array}$ \\
\hline ENSDARG00000024840 & ENSPART00000034417 & 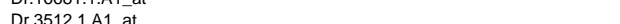 & OONWES BRAR & 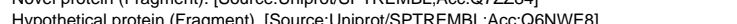 & $\begin{array}{lll} & \\
-12626\end{array}$ & 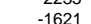 & 1 & 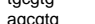 \\
\hline 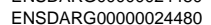 & ENSDART00000034117 & Dr.3512.1.A1 at & Q6NWE8BRARE & Hypothetical protein (Fragment). [Source:Uniprot/SPTREMBL;ACc:Q6NWE8] & -1593 & -1588 & -1 & 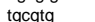 \\
\hline ENSDARG000000024480 & ENSDARTO00000034117 & Dr.3512.1.A1 at & Q6NWE8BRARE & & -1573 & -1568 & -1 & tgcgta \\
\hline ENSDARG00000024480 & ENSDART00000034117 & Dr.3512.1.A1 at & Q6NWEB BRARE & Hypothetical protein (Fragment). [Source:Uniprot/SPTREMBL;ACc:Q6NWE8] & -1569 & -1564 & -1 & tgcotg \\
\hline ENSDARG000000024480 & ENSDART00000034117 & 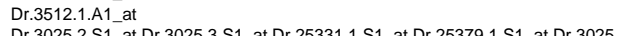 & Q6NWE8_BRARE & Hypothetical protein (Fragment). [Source:Uniprot/SPTREMBL;;Acc:Q6NWEE] & -1565 & -1560 & -1 & 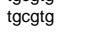 \\
\hline ENSDARG00000024892 & ENSDART00000033228 & $\begin{array}{l}\text { 2.S1_at,Dr.3025.3.S1_at,Dr.25379.1.S1_at,Dr.3025.1.A1_at,Dr.3025.3.S1_at } \\
\text { Dr.3025.2.S1_at,Dr.3025.3.S1_at,Dr.25331.1.S1_at,Dr.25379.1.S1_at,Dr.3025 }\end{array}$ & & & -3817 & -3812 & -1 & agcgtg \\
\hline ENSDARG00000024892 & ENSDART00000033228 & $\begin{array}{l}\text { 2.S1_at,Dr.3025.3.S1_at,Dr.25379.1.S1_at,Dr.3025.1.A1_at,Dr.3025.3.S1_at at } \\
\text { Dr.3025.2.S1_at,Dr.3025.3.S1__at,Dr.25331.1.S1__at,Dr.25379.1.S1_at,Dr.3025 }\end{array}$ & & & -3698 & -3693 & -1 & agcgtg \\
\hline ENSDARG00000024892 & ENSDART00000033228 & $\begin{array}{l}\text {.2.S1_at,Dr.3025.3.S1_at,Dr.25379.1.S1_at,Dr.3025.1.A1_at,Dr.3025.3.S1_at } \\
\text { Dr.3025.2.S1_at,Dr.3025.3.S1_at,Dr.25331.1.S1_at,Dr.25379.1.S1_at,Dr.3025 }\end{array}$ & & & -2476 & -2471 & -1 & cgcgtg \\
\hline ENSDARG00000024892 & ENSDART00000033228 & $\begin{array}{l}\text {.2.S1_at,Dr.3025.3.S1_at,Dr.25379.1.S1_at,Dr.3025.1.A1_at,Dr.3025.3.S1_at } \\
\text { Dr.3025.2.S1_at,Dr.3025.3.S1_at,Dr.25331.1.S1_at,Dr.25379.1.S1_at,Dr.3025 }\end{array}$ & & & -4576 & -4571 & 1 & ggcgtg \\
\hline ENSDARG00000024892 & ENSDART00000033228 & $\begin{array}{l}\text {.2.S1_at,Dr.3025.3.S1_at,Dr.25379.1.S1_at,Dr.3025.1.A1_at,Dr.3025.3.S1_at } \\
\text { Dr.3025.2.S1_at,Dr.3025.3.S1_at,Dr.25331.1.S1_at,Dr.25379.1.S1_at,Dr.3025 }\end{array}$ & & & -4314 & -4309 & 1 & agcgtg \\
\hline ENSDARG00000024892 & ENSDART00000033228 & .2.S1_at,Dr.3025.3.S1_at,Dr.25379.1.S1_at,Dr.3025.1.A1_at,Dr.3025.3.S1_at & & & -4209 & -4204 & 1 & tgcgtg \\
\hline
\end{tabular}


ENSDARG00000024892 ENSDART00000061058

ENSDARG00000024892 ENSDART00000061058

ENSDARG00000024892 ENSDART00000033228

ENSDARG00000024892 ENSDART00000033228

ENSDARG00000024892

ENSDART00000033228

ENSDARG00000024892

ENSDARG00000024892

ENSDARG00000024892

ENSDARG00000024892

ENSDARG00000024892 ENSDARG00000025147
ENSDARG00000025147 ENSDARG00000025147 ENSDARG00000025147 ENSDARG00000025147 ENSDARG00000025147
ENSDARG00000025254 ENSDARG00000025400 ENSDARG00000025400 ENSDARG00000025400 ENSDARG00000025400 ENSDARG00000025400 ENSDARG00000025400 ENSDARG00000025400 ENSDARG00000025400 ENSDARG00000025400 ENSDARG00000025400
ENSDARG00000026039 ENSDARG00000026039
ENSDARG00000026039 ENSDARG00000026039 ENSDARG00000026039
ENSDARG00000026039 ENSDARG00000026039 ENSDARG 00000026039
ENSDARG 00000026039 ENSDARG00000026039 ENSDARG00000026039 ENSDARG00000026039
ENSDARG00000026039 ENSDARG00000026039 ENSDARG 000000026090
ENSDARG00000026090 ENSDARG 000000026090
ENSDARG00000026090 ENSDARG000000026090 ENSDARG000000226090
ENSDARG00000026680 ENSDARG00000026680 ENSDARG00000026680 ENSDARG000000026680 ENSDARG00000026680 ENDDARG 000000026680
ENSDARG00000026880 ENSDARG00000026726
ENSDARG00000026726

ENSDARTO00000031047

ENSDART00000031047

ENSDART0000004486

ENSDART00000044869

ENSDART00000046418

ENSDART0000004641

ENSDART00000061150

ENSDARTO000006115

ENSDART00000061161

ENSDART0000006116

ENSDART00000061165

ENSDARTO000006116

ENSDARTT0000003820

ENSDART0000003820

ENSDARTO0000003820

ENSDART0000003820

ENSDART0000003820

ENSDARTO000003820

ENSDARTO0000003820

ENSDARTO00000038200

ENSDARTO00000035302

ENSDARTO0000035302

ENSDART00000035302

ENSDART00000035302

ENSDART0000003660

ENSDART00000003660

ENSDART0000003660

ENSDARTT00000003660

ENSDARTO000003660

ENSDARTT00000036600

ENSDART00000031665

ENSDART0000003166
ENSDARTO000003166 Symbor Description

2.S1_at,Dr.3025.3.S1_at,Dr.25379.1.S1_at,Dr.3025.1.A1_at,Dr.3025.3.S1_at
Dr.3025.2.S1_at,Dr.3025.3.S1_at,Dr.25331.1.S1_at,Dr.25379.1.S1_at,Dr.3025

\begin{tabular}{|c|c|c|c|}
\hline -1600 & -1595 & -1 & tgcgtg \\
\hline-4093 & -4088 & -1 & ggcgtg \\
\hline-3817 & -3812 & -1 & agcgtg \\
\hline-3698 & -3693 & -1 & agcgtg \\
\hline-2476 & -2471 & -1 & cgcgtg \\
\hline-4576 & -4571 & 1 & ggcgtg \\
\hline-4314 & -4309 & 1 & agctgtg \\
\hline-4209 & -4204 & 1 & tgcgtg \\
\hline -1600 & -1595 & -1 & tgcgtg \\
\hline $\begin{array}{l}-4093 \\
-3469\end{array}$ & $\begin{array}{l}-4088 \\
-3464\end{array}$ & $\begin{array}{l}-1 \\
-1\end{array}$ & $\begin{array}{l}\text { ggcgtg } \\
\text { agcotg }\end{array}$ \\
\hline-3453 & -3448 & 1 & ggctg \\
\hline $\begin{array}{l}-3028 \\
-30000\end{array}$ & $\begin{array}{l}-3023 \\
-29995\end{array}$ & $\begin{array}{l}-1 \\
-1\end{array}$ & $\begin{array}{c}\text { cgctgtg } \\
\text { ccacta }\end{array}$ \\
\hline-3000 & $\begin{array}{r}-2995 \\
-2955\end{array}$ & -1 & cgcgtg \\
\hline-2960 & $\begin{array}{l}-2955 \\
-2658\end{array}$ & -1 & cgcgtg \\
\hline-2645 & -2640 & 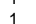 & $\begin{array}{l}\text { tgcgrg } \\
\text { tagcato }\end{array}$ \\
\hline-2555 & $\begin{array}{l}-2040 \\
-2550\end{array}$ & $\begin{array}{l}1 \\
1\end{array}$ & $\begin{array}{l}\text { cgcgtg } \\
\text { col }\end{array}$ \\
\hline-2330 & -2325 & 1 & agcgtg \\
\hline-2534 & -2529 & 1 & tgcgtg \\
\hline-652 & -647 & 1 & tgcgtg \\
\hline-2534 & -2529 & 1 & tgcgtg \\
\hline-652 & -647 & 1 & tgcgtg \\
\hline-2633 & -2628 & 1 & tgcgtg \\
\hline-751 & -746 & 1 & tgcgtg \\
\hline-2534 & -2529 & 1 & tgcgtg \\
\hline-652 & -647 & 1 & tgcgtg \\
\hline-2534 & -2529 & 1 & tgcgtg \\
\hline & -047 & 1 & $\operatorname{tgcg}$ \\
\hline-3607 & -3602 & 1 & ggcgtt \\
\hline-3509 & -3504 & 1 & tgcgtg \\
\hline-2574 & -2569 & -1 & tgcgtg \\
\hline $\begin{array}{l}-2532 \\
-2503\end{array}$ & $\begin{array}{l}-2527 \\
-2498\end{array}$ & -1 & cgcgtg \\
\hline $\begin{array}{l}-2503 \\
-2383\end{array}$ & $\begin{array}{l}-2498 \\
-2378\end{array}$ & -1 & agctgtg \\
\hline-2367 & -2362 & -1 & $\begin{array}{l}\text { cgcgtg } \\
\text { cgats }\end{array}$ \\
\hline-2270 & -2265 & -1 & tgcgtg \\
\hline-2238 & -2233 & -1 & cgcgtg \\
\hline-2201 & -2196 & 1 & cgcgtg \\
\hline $\begin{array}{r}-4632 \\
-461\end{array}$ & $\begin{array}{l}-4627 \\
-456\end{array}$ & $\begin{array}{c}1 \\
-1\end{array}$ & $\begin{array}{c}\text { cgctg tg } \\
\text { tgcctg }\end{array}$ \\
\hline-4546 & -4541 & $\begin{array}{c}-1 \\
1\end{array}$ & $\begin{array}{l}\text { tggerg } \\
\text { tgcgtg }\end{array}$ \\
\hline-4538 & -4533 & , & tgcgtg \\
\hline-364 & -359 & -1 & ggcgtg \\
\hline-222 & -217 & 1 & tgcgtg \\
\hline -3919 & -3914 & -1 & tgcgtg \\
\hline $\begin{array}{l}-4467 \\
-467\end{array}$ & & 1 & cgcgtg \\
\hline $\begin{array}{l}-4403 \\
-4459\end{array}$ & $\begin{array}{l}-4430 \\
-445\end{array}$ & 1 & tgcgtg \\
\hline-4154 & -4149 & 1 & $\begin{array}{l}\text { tgggrg } \\
\text { cgcgtat }\end{array}$ \\
\hline-3320 & -3315 & 1 & ggcgtt \\
\hline-3147 & -3142 & -1 & agcgtg \\
\hline $\begin{array}{l}-1685 \\
-917\end{array}$ & $\begin{array}{l}-1680 \\
-912\end{array}$ & $\begin{array}{c}1 \\
-1\end{array}$ & $\begin{array}{l}\text { agctg } \\
\text { tgcctg }\end{array}$ \\
\hline-401 & -396 & 1 & $\begin{array}{l}\text { tgcgtg } \\
\text { tgeng }\end{array}$ \\
\hline-3320 & -3315 & 1 & ggcgtg \\
\hline-3147 & -3142 & 1 & agctg \\
\hline - 1685 & -1680 & 1 & agcgtg \\
\hline 401 & $\begin{array}{l}-912 \\
-996\end{array}$ & -1 & $\begin{array}{l}\text { tgcgtg } \\
\text { tgcatg }\end{array}$ \\
\hline 117 & -112 & 1 & cgcgtg \\
\hline-4436 & -4431 & -1 & agcgtg \\
\hline
\end{tabular}

Cd63 antigen [Source:RefSeq_peptide;Acc:NP_955837]

Cd63 antigen [Source:RefSeq_peptide;ACAc:NP__955837]

Cd63 antigen [Source:RefSeq_peptide;Acc:NP 955837

Cd63 antigen [Soure.Req_epeptde,Ac.NP_-955837

Cd63 antigen [Source:RefSeq_peptide;Acc:NP ${ }^{-955837}$

Cd63 antigen [Source:RefSeq_peptide;AAcc:NP_95583]

2.S1_at,Dr.3025.3.S1_at,Dr.25379.1.S1_at,Dr.3025.1.A1_at,Dr.3025.3.S1_at
Dr.3025.2.S1_at,Dr.3025.3.S1_at,Dr.25331.1.S1_at,Dr.25379.1.S1_at,Dr.3025

2.S1_at,Dr.3025.3.S1_at,Dr.25379.1.S1_at,Dr.3025.1.A1_at,Dr.3025.3.S1_at
Dr.3025.2.S1_at,Dr.3025.3.S1_at,Dr.25331.1.S1_at,Dr.25379.1.S1_at,Dr.302

2.S1_at,Dr.3025.3.S1_at,Dr.25379.1.S1_at,Dr.3025.1.A1_at,Dr.3025.3.S1_at
Dr.3025.2.S1_at,Dr.3025.3.S1_at,Dr.25331.1.S1_at,Dr.25379.1.S1_at,Dr.3025

.2.S1_at,Dr.3025.3.S1_at,Dr.25379.1.S1_at,Dr.3025.1.A1_at,Dr.3025.3.S1_at
Dr.3025.2.S1_at,Dr.3025.3.S1_at,Dr.25331.1.S1_at,Dr.25379.1.S1_at,Dr.3025

.2.S1_at_Dr.3025.3.S1_at,Dr.25379.1.S1_at_,Dr.3025.1.A1_at,Dr.3025.3.S1_at
Dr.3025.2.S1_at,Dr.3025.3.S1_at,Dr.25331.1.S1_at,Dr.25379.1.S1_at,Dr.3025

2.S1_at,Dr.3025.3.S1_at,Dr.25379.1.S1_at,Dr.3025.1.A1_at,Dr.3025.3.S1_at
Dr.3025.2.S1_at,Dr.3025.3.S1_at,Dr.25331.1.S1_at,Dr.25379.1.S1_at,Dr.3025

Dr.3459.1.S1_at

Dr.3459.1.S1_at

Dr.3459.1.S1_at

Dr.3459.1.S1_at
Dr.1201.1.S1_at

Dr.25009.4.A1_at,Dr.25009.4.A1_at,Dr.25009.4.A1_at,Dr.25009.4.A1_at,Dr.25 009.4.A1_at_.at._25009.4.A1_at,Dr.25009.4.A1_at,Dr.25009.4.A1_at,Dr.25009.4.A1_at,Dr.25 009.4.A1_at
Dr.25009.4.A1_at,Dr.25009.4.A1_at,Dr.25009.4.A1_at,Dr.25009.4.A1_at,Dr.25 009.4.A1_at
Dr.25009.4.A1_at,Dr.25009.4.A1_at,Dr.25009.4.A1_at,Dr.25009.4.A1_at,Dr.25 D09.4.A1_at 25009.4.A1_at,Dr.25009.4.A1_at,Dr.25009.4.A1_at,Dr.25009.4.A1_at,Dr.25 Dr.25009.4.A1_at,Dr.25009.4.A1_at,Dr.25009.4.A1_at,Dr.25009.4.A1_at,Dr.25 Dr.25009.4.A1_at,Dr.25009.4.A1_at,Dr.25009.4.A1_at,Dr.25009.4.A1_at,Dr.25 009.4.A1_at Dr.25009_.4.A1_at,Dr.25009.4.A1_at,Dr.25009.4.A1_at,Dr.25009.4.A1_at,Dr.25 009.4.A1_at_at_Dr.25009.4.A1_at,Dr.25009.4.A1_at,Dr.25009.4.A1_at,Dr.25 Dr.25009.A1_at,Dr.25009.4.A1_at,Dr.25009.4.A1_at,Dr.25009.4.A1_at,Dr. 25 009.4.A1_at

Dr.9478.1.S1_at
Dr.9478. 1 at

D.9478.1.S1 at

Dr.9478.1.S1_at

Dr.9478.1.S1_a

Dr.9478.1.S1_at
Dr.9478.1.S1 at

Dr.9478.1.S1_at

Dr.9478.1.S1_at

Dr.9478.1.S1_at
Dr.9478.1.S1_at

Dr.9478.1.S1_at

Dr.9478.1.S1_at

Dr.9478.1.S1_aa
Dr.9478.1.S1 at
D.

Dr.9478.1.S1-at

Dr.12833.1.A1-at
Dr.128331.A1 at
D.

Dr.12833.1.A1_at
Dr.12833.1.A1_at

Dr.26339.1.A1_at

Dr.26139.1.A1_at,Dr.26139.1.A1_-

Dr.26139.1.A1_at,Dr.26139.1.A1_x a

Dr.26139.1.A1_at,Dr.26139.1.A1_X_al

Dr.26139.1.A1_at,Dr.26139.1.A1_x_a

Dr.26139.1.A1_at,Dr.26139.1.A1_x_at

Dr.269.1.1.1._at
Dr.59.1.S1_at

cyp1a
cyp1a
cyp1a
cyp1a
cyp1a
cyp1a
cyp1a
cyp1a
cyp1a
cyp1a
cyp1a
cyp1a
cyp1a
cyp1a
cyp1a
cyp1a
zgc:64213
zgc:64213
zgc:64213
zgc:64213
zgc:64213
slc25a14
slc25a14
slc25a14
slc25a14
slc25a14
slc25a14
sl22514
slc25a14
slc25a14
slc25a14
anxa1a
anxa1a

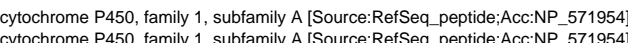
cytochrome P450, family 1, subtamily A A Source-RefSec peptide.Acc:NP 571954 cyttochrome P450, family 1, subfamily A [Source:RefSeq_peptide;ACc:NP_571954 cytochrome P450, family 1, subtamily A Source:RefSeq_peptide;Acc:NP_571954

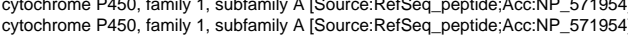
cytochrome P450, family 1, subfamily A [Source:RefSeq_peptide;Acc:NP 571954 cytochrome P450, family 1, subfamily A [Source:RefSeq_peptide;ACc:NP_ 571954 cytochrome P450, family 1, subfamily A Source:RefSeq_peptide;AAcc:NP_571954
cytochrome P450, family 1, subfamily A ISource:RefSeq_ peptide:Acc:NP 571954 cytochrome P450, family 1, subfamily A ISource:RefSeq peptide;Acc:NP 571954 cytochrome P450, family 1, subfamily A [Source:RefSeq_peptide;Acc:NP-571954

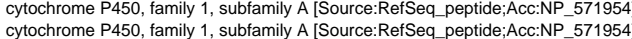
cytochrome P450, family 1 , subfamily A [Source:RefSeq peptide:Acc:NP 571954 hypothetical protein LOC393393 [Source:RefSeq_peptide;ACC:NP_-956715] hypothetical protein LOC 393393 3 Source:RefSeq_peptide;AAC:NPP-95671 hypothetical protein LOC 393393 3 Source:RefSeq peptide:ACC:NP 956719 hypothetical protein LOC393393 [Source:RefSeq_peptide;ACc:NP_956715] solute carrier family 25 (mitochondrial carrier, brain), member 14 [Source.RetSeq_peptide;Acc:NP_95645 solute carrier family 25 (mitochondrial carrier, brain), member 14 [Source:RefSeq_peptidd;AAcc:NP-956458
solute carrier family 25 (mitochondrial carrier, brain), member 14 [Source:RefSeq_peptide;Acc:NP-95645 solute carrier family 25 (mitochondrial carrier, brain), member 14 [Source:RetSeq_peptide,Acc.NPB-95645 solute carrier family 25 (mitochondrial carrier, brain), member 14 [Source:RefSeq_peptide;Acc:NP_95645

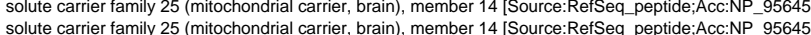
olute carrier family 25 (mitochondrial carrier, brain) member 14 Source RetSeq peptide.Acc.NP 95645 solute carrier family 25 (mitochondrial carrier, brain), member 14 [Source:Reffeq_peptide:Acc:NP__95645

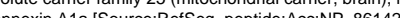
annexin A1a [Source:RefSeq_peptide;Acc:NP_861423]
annexin A1a [Source:RefSeq_peptide;Acc:NP_861423]

Page 4 


\begin{tabular}{|c|c|c|c|c|c|c|c|c|}
\hline $\begin{array}{l}\text { Supplementary Table } 2 \text { (c } \\
\text { Gene ID }\end{array}$ & $\begin{array}{l}\text { tit.). Location of putative } A \\
\text { Transcript ID }\end{array}$ & Affy IDs & Symbol & & Start & End & Strand & Sequence \\
\hline ENSDARG00000027088 & ENSDART00000039267 & Dr.1192.1.S1_at,Dr.1192.1.S1_a_at & $\begin{array}{ll}\text { Symbol } \\
\text { wu:fb11h03 }\end{array}$ & $\begin{array}{l}\text { Description } \\
\text { prostaglandin D2 synthase [Source:RefSeq peptide;ACc:NP 998799] }\end{array}$ & Stat -4658 & End -4653 & Strana & tgcquentge \\
\hline ENSDARG00000027088 & ENSDART00000039267 & Dr.1192.1.S1__t,Dr.1192.1.S1_a_at & wu:fb11ho3 & prostaglandin D2 synthase [Source:RefSeq_peptide;ACC:NP_998799] & -166 & -161 & -1 & tgcgtg \\
\hline ENSDARG00000027088 & ENSDART00000039267 & Dr.1192.1.S1_at,Dr.1192.1.S1_a_at & wu:fb11h03 & prostaglandin D2 synthase [Source:RefSeq_peptide;Acc:NP_-998799] & -136 & -131 & 1 & tgcgtg \\
\hline ENSDARG00000027088 & ENSDART00000039267 & Dr.1192.1.S1_at,Dr.1192.1.S1_a_at & wu:fb11h03 & prostaglandin D2 synthase [Source:RefSeq_peptide;Acc:NP_-998799] & -4658 & -4653 & 1 & tgcgtg \\
\hline ENSDARG00000027088 & ENSDART00000039267 & Dr.1192.1.S1_at,Dr.1192.1.S1_a_at & wu:fb11ho3 & prostaglandin D2 synthase [Source:RefSeq_peptide;Acc:NP_998799] & -166 & -161 & -1 & tgctg \\
\hline $\begin{array}{l}\text { ENSDARG000000027088 } \\
\text { ENDSARG0000 }\end{array}$ & $\begin{array}{l}\text { ENSDART00000039267 } \\
\text { ENDAR }\end{array}$ & 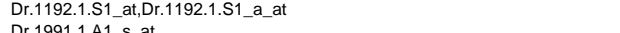 & 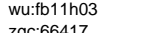 & prostaglandin D2 synthase [Source:RefSeq_peptide;Acc:NP_998799] & -136 & -131 & 1 & $\operatorname{tgcgtg}$ \\
\hline $\begin{array}{l}\text { ENSDARG000000027669 } \\
\text { ENSDARG00000027669 }\end{array}$ & $\begin{array}{l}\text { ENSDART00000035274 } \\
\text { ENSDART00000035274 }\end{array}$ & $\begin{array}{l}\text { Dr.1991.1.A1_s_at } \\
\text { Dr.1991.1.A1_s_at }\end{array}$ & $\begin{array}{l}\text { zgc:66417 } \\
\text { zgc:66417 }\end{array}$ & $\begin{array}{l}\text { hypothetical protein LOC387303 [Source:RefSeq_peptide;Acc:NP_956419] } \\
\text { hypothetical protein LOC387303 [Source:RefSeq_peptide;Acc:NP_956419] }\end{array}$ & $\begin{aligned}-2874 \\
-129\end{aligned}$ & $\begin{array}{r}-2869 \\
-124\end{array}$ & $\begin{array}{c}1 \\
-1\end{array}$ & $\begin{array}{l}\text { tgcgtg } \\
\text { ggcgtg }\end{array}$ \\
\hline & & Dr.25593.2.A1__at,Dr.25593.1.S1_at,Dr.4412.12.A1_at,Dr.25593.2.A1_at,Dr.25 & & & & & & \\
\hline ENSDARG00000027924 & ENSDART00000024900 & 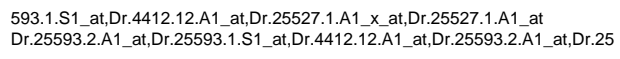 & & & -3372 & -3367 & -1 & agcgtg \\
\hline ENSDARG00000027924 & ENSDART00000024900 & $\begin{array}{l}\text { 593.1.S1_at,Dr.4412.12.A1_at,Dr.25527.1.A1_x_at,Dr.25527.1.A1_at } \\
\text { rr.25593.2.A1_at,Dr.25593.1.S1_at,Dr.4412.12.A1_at,Dr.25593.2.A1_at,Dr.25 }\end{array}$ & & & -2691 & -2686 & -1 & agcgtg \\
\hline ENSDARG00000027924 & ENSDART00000024900 & $\begin{array}{l}\text { 593.1.S1_at,Dr.4412.12.A1_at,Dr.25527.1.A1 x at,Dr.25527.1.A1_at } \\
\text { Dr.25593.2.A1_at,Dr.25593.1.S1_at,Dr.4412.122.A1_at,Dr.25593.2.A1_at,Dr.25 }\end{array}$ & & & -1910 & -1905 & 1 & tgcgtg \\
\hline ENSDARG00000027924 & ENSDART00000024900 & $\begin{array}{l}\text { 593.1.S1_at,Dr.4412.12.A1_at,Dr.25527.1.A1_x_at,Dr.25527.1.A1_at } \\
\text { rr.25593.2.A1_at,Dr.25593.1.S1_at,Dr.4412.12.A1_at,Dr.25593.2.A1_at,Dr.25 }\end{array}$ & & & -4427 & -4422 & 1 & cgcgtg \\
\hline ENSDARG00000027924 & ENSDART00000047994 & $\begin{array}{l}\text { 593.1.S1_at,Dr.4412.12.A1_at,Dr.25527.1.A1_x_at,Dr.25527.1.A1_at } \\
\text { rr.25593.2.A1_at,Dr.25593.1.S1_at,Dr.4412.12.A1_at,Dr.25593.2.A1_at,Dr.25 }\end{array}$ & & & -4195 & -4190 & 1 & tgcgtg \\
\hline ENSDARG00000027924 & ENSDART00000024900 & 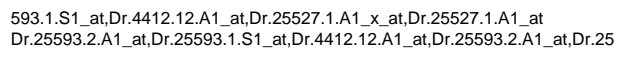 & & & -3372 & -3367 & -1 & agcgtg \\
\hline ENSDARG00000027924 & ENSDART00000024900 & $\begin{array}{l}\text { 593.1.S1_at,Dr.4412.12.A1_at,Dr.25527.1.A1_x_at,Dr.25527.1.A1_at } \\
\text { rr.25593.2.A1_at,Dr.25593.1.S1_at,Dr.4412.12.A1_at,Dr.25593.2.A1_at,Dr.25 }\end{array}$ & & & -2691 & -2686 & -1 & agcgtg \\
\hline ENSDARG00000027924 & ENSDART00000024900 & $\begin{array}{l}\text { 593.1.S1_at,Dr.4412.12.A1_at,Dr.25527.1.A1_x_at,Dr.25527.1.A1_at } \\
\text { rr.25593.2.A1_at,Dr.25593.1.S1_at,Dr.4412.12.A1_at,Dr.25593.2.A1_at,Dr.25 }\end{array}$ & & & -1910 & -1905 & 1 & tgcgtg \\
\hline ENSDARG00000027924 & ENSDART00000024900 & 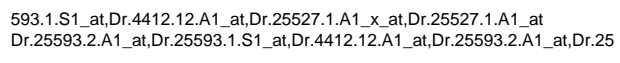 & & & -4427 & -4422 & 1 & cgcgtg \\
\hline ENSDARG00000027924 & ENSDART00000047994 & 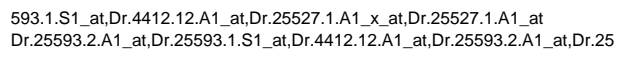 & & & -4195 & -4190 & 1 & tgcgtg \\
\hline ENSDARG00000027924 & ENSDART00000024900 & 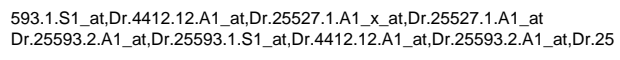 & & & -3372 & -3367 & -1 & agcgtg \\
\hline ENSDARG00000027924 & ENSDART00000024900 & 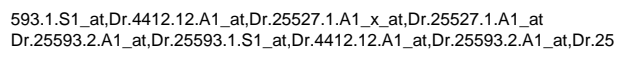 & & & -2691 & -2686 & -1 & agcgtg \\
\hline ENSDARG00000027924 & ENSDART00000024900 & $\begin{array}{l}\text { 593.1.S1_at,Dr.4412.12.A1_at,Dr.25527.1.A1_x_at,Dr.25527.1.A1_at } \\
\text { rr.25593.2.A1_at,_r.25593.1.S1_at,Dr.4412.12.A1_at,Dr.25593.2.A1_at,Dr.25 }\end{array}$ & & & -1910 & -1905 & 1 & tgcgtg \\
\hline ENSDARG00000027924 & ENSDART00000024900 & 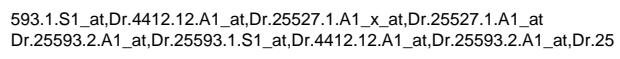 & & & -4427 & -4422 & 1 & cgcgtg \\
\hline ENSDARG00000027924 & ENSDART00000047994 & 593.1.S1_at,Dr.4412.12.A1_at,Dr.25527.1.A1_X_at,Dr.25527.1.A1_at & & & -4195 & -4190 & 1 & tgcgtg \\
\hline ENSDARG000000028618 & ENSDARTO00000028260 & Dr.890.1.s1_at & zgc:77517 & hypothetical protein LOC393540 [Source:RefSeq_peptide;Acc:NP_956862] & -3818 & -3813 & -1 & $\operatorname{tgctg}$ \\
\hline $\begin{array}{l}\text { ENSDARG00000028618 } \\
\text { ENSDARG00000028784 }\end{array}$ & $\begin{array}{l}\text { ENSDART000000028260 } \\
\text { ENSDART00000044410 }\end{array}$ & $\begin{array}{l}\text { Dr.890.1.S1_at } \\
\text { Dr.2714.A1 at }\end{array}$ & zgc:77517 & hypothetical protein LOC393540 [Source:RefSeq_Peptide;ACA:NP_956862] & -102 & -97 & 1 & cgcgtg \\
\hline $\begin{array}{l}\text { ENSDARG000000028284 } \\
\text { ENSDARG0000029086 }\end{array}$ & & $\begin{array}{l}\text { D.r.212714.1.A1_at } \\
\text { Dr.1282.1.11at }\end{array}$ & krt8 & & $\begin{array}{r}-551 \\
-3387\end{array}$ & $\begin{array}{r}-546 \\
-3832\end{array}$ & -1 & agctg \\
\hline ENSDARG00000030236 & ENSDART00000011928 & Dr.1368.7.S1_at & $\begin{array}{l}\text { KII } \\
\text { zgc:91877 }\end{array}$ & 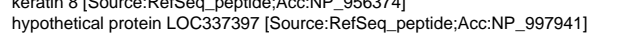 & -1028 & -1023 & -1 & tgcgtg \\
\hline ENSDARG000000030236 & ENSDART00000011928 & Dr.1368.7.S1_at & zgc:91877 & hypothetical protein LOC337397 [Source:RefSeq_peptide;Acc:NP_997941] & 77 & 82 & -1 & agcgtg \\
\hline ENSDARG000000030662 & ENSDART00000048550 & Dr.9682.1.A1_at & & & -3682 & -3677 & 1 & $\operatorname{tgcgtg}$ \\
\hline $\begin{array}{l}\text { ENDARGG00000030062 } \\
\text { ENSDARG00000030662 }\end{array}$ & $\begin{array}{l}\text { ENSDART000000048550 } \\
\text { ENSDART0000048550 }\end{array}$ & Dr.9682.1.A1_at & & & $\begin{array}{l}-1444 \\
-301\end{array}$ & $\begin{array}{l}-1439 \\
-296\end{array}$ & $\begin{array}{c}1 \\
-1\end{array}$ & cgcgtg \\
\hline $\begin{array}{l}\text { ENSSARG000000030662 } \\
\text { ENSDARG00000030662 }\end{array}$ & $\begin{array}{l}\text { ENSDART000000048550 } \\
\text { ENSDART0000048550 }\end{array}$ & $\begin{array}{l}\text { Dr.9682.1.A1_at } \\
\text { D.r.6682.1.A1 at }\end{array}$ & & & $\begin{array}{r}-301 \\
-4904\end{array}$ & $\begin{array}{r}-296 \\
-499\end{array}$ & -1 & tgcgtg \\
\hline ENSDARG00000031664 & ENSDART00000049339 & $\begin{array}{l}\text { Dr.6682.1.A1_at at } \\
\text { Dr.8215.1.A_ at }\end{array}$ & sox21a & SRY-box 21 [Source:RefSeq peptide:Acc:NP 571361] & -2414 & -2409 & 1 & $\begin{array}{l}\text { agcgtg } \\
\text { agcig }-1\end{array}$ \\
\hline ENSDARG00000031664 & ENSDART00000049339 & Dr.8215.1.A1_at & sox21a & SRY-box 21 [Source:RefSeq_peptide;Acc:NP_571361] & -311 & -306 & -1 & ggctg \\
\hline ENSDARG00000031664 & ENSDART00000049339 & Dr.8215.1.A1_at & sox21a & SRY-box 21 [Source:RefSeq_peptide;Acc:NP_571361] & -213 & -208 & 1 & cgcgtg \\
\hline $\begin{array}{l}\text { ENSDARG000000031854 } \\
\text { NSDARG }\end{array}$ & $\begin{array}{l}\text { ENSDART00000043418 } \\
\text { ENDAR }\end{array}$ & Dr.7688.1.A1_at,Dr.7688.1.A1_at & & & -4616 & -4611 & -1 & $\operatorname{tgcgtg}$ \\
\hline $\begin{array}{l}\text { ENDDARG000000031854 } \\
\text { ENSDARG00000031854 }\end{array}$ & $\begin{array}{l}\text { ENSDART000000043418 } \\
\text { ENSDART0000043418 }\end{array}$ & 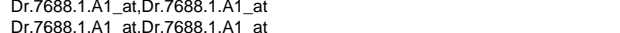 & & & $\begin{array}{l}-769 \\
-545\end{array}$ & $\begin{array}{l}-764 \\
-540\end{array}$ & $\begin{array}{c}1 \\
-1\end{array}$ & cgctg \\
\hline ENSDARG00000031854 & ENSDART00000050805 & 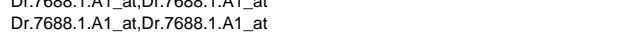 & & & $\begin{array}{l}-545 \\
-4616\end{array}$ & $\begin{array}{l}-540 \\
-4611\end{array}$ & -1 & $\begin{array}{l}\text { ggcigg } \\
\text { tgcgtg }-2\end{array}$ \\
\hline ENSDARG00000031854 & ENSDART00000050805 & Dr.7688.1.A1_at,Dr.7688.1.A1_at & & & -769 & -764 & 1 & cgcgtg \\
\hline ENSDARG00000031854 & ENSDART00000050805 & Dr.7688.1.A1_at,Dr.7688.1.A1_at & & & -545 & -540 & -1 & ggcgtg \\
\hline ENSDARG000000033770 & ENSDART00000011961 & Dr.25009.6.A1_a_at & $\mathrm{vg} 1$ & Vitellogenin 1 (Fragment). [Source:Uniprot/SPTREMBL;ACc:Q8JH36] & -3386 & -3381 & -1 & cgcgtg \\
\hline ENSDARG000000033770 & ENSDART00000011961 & Dr.25009.6.A1_a_at & vg1 & Vitellogenin 1 (Fragment). [Source:Uniprot/SPTREMBL;Acc:Q8JH36] & -2983 & -2978 & 1 & ggcgtg \\
\hline $\begin{array}{l}\text { ENSDARG000000033770 } \\
\text { ENSARG000003370 }\end{array}$ & $\begin{array}{l}\text { ENSDART00000011961 } \\
\text { ENSART0000011961 }\end{array}$ & Dr.25009.6.A1_a_at & vg1 1 & 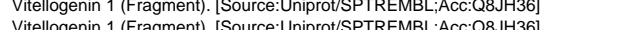 & -2703 & -2698 & -1 & $\operatorname{tgcgtg}$ \\
\hline $\begin{array}{l}\text { ENSDARG000000033770 } \\
\text { ENSARG0000003770 }\end{array}$ & $\begin{array}{l}\text { ENSDART000000011961 } \\
\text { ENDART00000011961 }\end{array}$ & $\begin{array}{l}\text { Dr.2509.6.A1_a a at } \\
\text { Dr25009.6.A1 a at }\end{array}$ & $\begin{array}{l}\text { vg1 } \\
\text { vg1 }\end{array}$ & 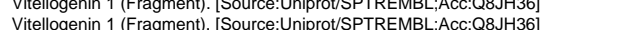 & -2417 & -2412 & -1 & tgcgtg \\
\hline $\begin{array}{l}\text { ENSDARG000000033770 } \\
\text { ENDARG0000033770 }\end{array}$ & $\begin{array}{l}\text { ENSDART000000011961 } \\
\text { ENDDART000001961 }\end{array}$ & $\begin{array}{l}\text { Dr.25090.6.A1_a a at } \\
\text { Dr.25009. A1 a at }\end{array}$ & $\begin{array}{l}\text { vg1 } \\
\text { vg1 }\end{array}$ & 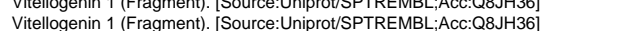 & $\begin{array}{l}-2381 \\
-1870\end{array}$ & $\begin{array}{l}-2376 \\
-1665\end{array}$ & 1 & tgcgttg \\
\hline $\begin{array}{l}\text { ENDSARG000000033770 } \\
\text { ENSDARG00000033770 }\end{array}$ & $\begin{array}{l}\text { ENSAR I0000011961 } \\
\text { ENSDART000001961 }\end{array}$ & Dr.25009.6.A1_a_at at & vg1 & Vitellogenin 1 (Fragment). (Source:Uniprot/SPTREMBL:ACc:OBJH36] & $\begin{array}{l}-1870 \\
-4407\end{array}$ & $\begin{array}{l}-1865 \\
-4402\end{array}$ & -1 & $\begin{array}{l}\text { tgggtg } \\
\text { gagctg }\end{array}$ \\
\hline $\begin{array}{l}\text { ENDDARG00000033770 } \\
\text { ENSDARG00000033770 }\end{array}$ & ENSDART00000011961 & Dr.25009.6.A1 a at & vg1 & Vitellogenin 1 (Fragment). [Source:Uniprot/SPTREMBL;Acc:O8JH36] & -4306 & -4301 & 1 & $\begin{array}{l}\text { cgcglig } \\
\text { cgctg }\end{array}$ \\
\hline ENSDARG00000033949 & ENSDART00000045135 & Dr.18466.1.A1_at & NP_001017680.1 & hypothetical protein LOC550375 [Source:RefSeq_peptide;AcC:NP_001017680] & -3737 & -3732 & 1 & tgcgtg \\
\hline ENSDARG00000033949 & ENSDART00000045135 & Dr.18466.1.A1_at & NP_001017680.1 & hypothetical protein LOC550375 [Source:RefSeq_peptide;ACC:NP_001017680] & -1464 & -1459 & 1 & agcgtg \\
\hline ENSDARG000000034351 & ENSDART00000043771 & Dr.2363.1.S1_at & $\operatorname{tag} \ln 2$ & transgelin 2 [Source:RefSeq_peptide;Acc:NP_963870] & -1492 & -1487 & 1 & agcgtg \\
\hline ENSDARG000000035160 & ENSDART00000050933 & Dr.13775.1.S1_at & & & -40 & -35 & 1 & agcgtg \\
\hline ENSDARG000000035666 & ENSDART00000051733 & Dr.4797.1.S1_at & Q6DGW1_BRARE & Hypothetical protein (Fragment). [Source:Uniprot/SPTREMBL;Acc:Q6DGW1] & -3118 & -3113 & -1 & ggcgtg \\
\hline ENSDARG00000035666 & ENSDART00000051733 & $\begin{array}{l}\text { Dr.4797.1.S1_at } \\
\text { Dr. } 74971 \text { s1 at }\end{array}$ & Q6DGW1_BRARE & Hypothetical protein (Fragment). [Source:Uniprot/SPTREMBL;Acc:Q6DGW1] & -3045 & -3040 & 1 & agcgtg \\
\hline $\begin{array}{l}\text { ENSDARG00000035666 } \\
\text { ENSDARG0000035666 }\end{array}$ & ENSDART00000051733 & $\begin{array}{l}\text { D.r.49.1.S1_at } \\
\text { Dr.4797.1.S1_at }\end{array}$ & $\begin{array}{l}\text { QDOWWI__RARE } \\
\text { Q6DGWIBRARE }\end{array}$ & 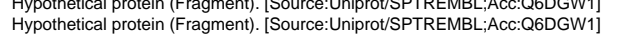 & ${ }_{-859}^{-850}$ & ${ }_{-854}^{-1401}$ & 1 & $\begin{array}{l}\text { ggcgtg } \\
\text { tgcgtg }\end{array}$ \\
\hline ENSDARG000000035743 & & $\begin{array}{l}\text { Dr.14748.1.S1_at } \\
\text { Dis }\end{array}$ & ZgC: 63985 & 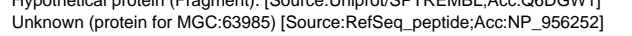 & -1043 & -1038 & 1 & agcgtg \\
\hline ENSDARG00000036082 & ENSDART00000052385 & Dr.15819.1.A1_at,Dr.17776.1.A1_at & NP_001001843.1 & tryptophan hydroxylase 1, like [Source:RefSeq_peptide:Acc:NP_001001843] & -3632 & -3627 & 1 & tgcgtg \\
\hline ENSDARG000000036082 & ENSDART00000052385 & Dr.15819.1.A1_at,Dr.17776.1.A1_at & NP_001001843.1 & tryptophan hydroxylase 1, like [Source:RefSeq_peptide;ACc:NP__001001843] & -1852 & -1847 & 1 & agcgtg \\
\hline ENSDARG00000036082 & ENSDART00000052385 & & NP_-001001843.1 & tryptophan hydroxylase 1, like [Source:RefSeq_peptide;Acc:NP_001001843] & -1701 & -1696 & -1 & agcgtg \\
\hline
\end{tabular}




\begin{tabular}{|c|c|c|c|c|c|c|c|c|}
\hline $\begin{array}{l}\text { Supplementary } \\
\text { Gene ID }\end{array}$ & 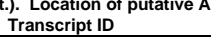 & & Symbol & Description & Start & End & Strand & Sequence \\
\hline $\begin{array}{l}\text { ENSDARG00000036082 } \\
\end{array}$ & ENSDART00000052385 & Dr.15819.1.A1_at,Dr.17776.1.A1_at & NP_001001843.1 & tryptophan hydroxylase 1, like [Source:RefSeq_peptide;Acc:NP_001001843] & & -235 & -1 & \\
\hline ENSDARG00000036082 & ENSDART00000052385 & Dr.15819.1.A1_at,Dr.17776.1.A1_at & NP_-001001843.1 & tryptophan hydroxylase 1, like [Source:RefSeq_peptide;Acc:NP_001001843] & -56 & -51 & 1 & cgcgtg \\
\hline ENSDARG00000036470 & ENSDART00000052991 & Dr.10326.1.S1_at & & jun B proto-oncogene [Source:RefSeq_peptide;Acc:NP_998721] & -66 & -61 & -1 & ggcgtg \\
\hline ENSDARG00000036481 & ENSDART00000053001 & Dr.6550.1.A1_at & Q6PG35_BRARE & LOC407646 protein (Fragment). [Source:Uniprot/SPTREMBL;ACC:Q6PG35] & -4651 & -4646 & -1 & tgcgtg \\
\hline ENSDARG00000036517 & ENSDART00000053070 & Dr.1411.1.S1_at & si:xx-by1文7g17.1 & Novel protein similar to zebrafish hemoglobin alpha-adult 1 (Hbaa1). [Source:Uniprot/SPTREMBL;Acc:Q6ZM17] & -2107 & -2102 & -1 & tgcgtg \\
\hline ENSDARG00000036517 & ENSDART00000053070 & Dr.1411.1.S1_at & si:xx-by187g17.1 & Novel protein similar to zebrafish hemoglobin alpha-adult 1 (Hbaa1). [Source:Uniprot/SPTREMBL;AAcc:Q6ZM17] & -4586 & -4581 & 1 & $\operatorname{tgcgtg}$ \\
\hline $\begin{array}{l}\text { ENSDARG000000036518 } \\
\text { ENSDARG000003518 }\end{array}$ & $\begin{array}{l}\text { ENSDART00000043748 } \\
\text { ENSDART0000043748 }\end{array}$ & Dr.25155.1.S1_s_at & ba2l & Hemoglobin beta-1 chain (BetaA1 globin). [Source:Uniprot/SWISSPROT;Acc:Q90486] & -3361 & -3356 & 1 & $\operatorname{tgcgtg}$ \\
\hline $\begin{array}{l}\text { ENSDARG000000036518 } \\
\text { ENSDARG00000036518 }\end{array}$ & $\begin{array}{l}\text { ENSDART000000043748 } \\
\text { ENSDART00000043748 }\end{array}$ & $\begin{array}{l}\text { Dr.25155.1.S1_s_at } \\
\text { Dr.25155.1.1 } 1 \text { sat }\end{array}$ & $\begin{array}{l}\text { ba21 } \\
\text { ba21 }\end{array}$ & 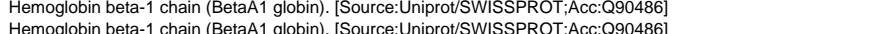 & -1341 & $\begin{array}{l}-1336 \\
-476\end{array}$ & -1 & tgcgtg \\
\hline ENSDARG00000036519 & $\begin{array}{l}\text { ENSDART000000043748 } \\
\text { ENSDART00000053072 }\end{array}$ & $\begin{array}{l}\text { DD.251555.1.S1__s_at } \\
\text { Dr.1411.1.S1_at }\end{array}$ & $\begin{array}{l}\text { ba2l } \\
\text { hbaa1 }\end{array}$ & 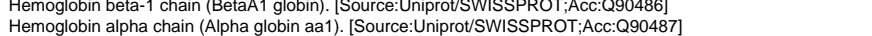 & $\begin{array}{l}-481 \\
-2950\end{array}$ & -2945 & $\begin{array}{c}-1 \\
1\end{array}$ & $\begin{array}{l}\operatorname{tgctg} \\
\text { cgctg }\end{array}$ \\
\hline ENSDARG00000036521 & ENSDART00000053073 & Dr.25155.1.S1_s_at & ba2 & Hemoglobin beta-1 chain (BetaA1 globin). [Source:Uniprot/SWISSPROT;ACc:Q90486] & -3811 & -3806 & 1 & tgcgtg \\
\hline & ENSDART00000053073 & Dr.25155.1.s1_s_at & ba2 & Hemoglobin beta-1 chain (BetaA1 globin). [Source:Uniprot/SWISSPROT;Acc:Q90486] & -1332 & -1327 & -1 & tgcgtg \\
\hline ENSDARG000000036527 & ENSDART00000053078 & Dr.1411.1.S1_at & si:xx-by187g17.1 & hemoglobin alpha adult-1 (hbaa1), mRNA [Source:RefSeq_dna;Acc:NM_131257] & -2104 & -2099 & -1 & tgcgtg \\
\hline ENSDARG000000036527 & ENSDART00000053078 & Dr.1411.1.S1_at & si:xx-by187g17.1 & hemoglobin alpha adult-1 (hbaa1), mRNA [Source:RetSeq_dna;ACc:NM_131257] & -4583 & -4578 & 1 & tgcgtg \\
\hline $\begin{array}{l}\text { ENSDARG000000036528 } \\
\text { ENSDAR00000036528 }\end{array}$ & $\begin{array}{l}\text { ENSDART000000053080 } \\
\text { ENSDARTO0000053080 }\end{array}$ & $\begin{array}{l}\text { DD.8723.1.S1__at } \\
\text { Dr.8723.1.S1 at }\end{array}$ & $\begin{array}{l}\mathrm{NP}-001002461.1 \\
\mathrm{NP} 0000261.1\end{array}$ & zgc:92903 [Source:RefSeq_peptide;Acc:NP_001002461] & -1100 & -1095 & -1 & agcgtg \\
\hline $\begin{array}{l}\text { ENSDARG0000000335528 } \\
\text { ENSDARG00000036529 }\end{array}$ & $\begin{array}{l}\text { ENSDART } \\
\text { ENSDART000000053080 }\end{array}$ & $\begin{array}{l}\text { Dr.8723.1.S1 at } \\
\text { Dr.25155.1.S1 s at }\end{array}$ & $\begin{array}{l}\text { NPa2l } \\
\text { bat1002461.1 }\end{array}$ & 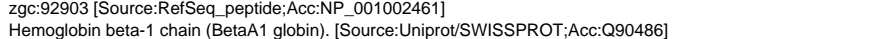 & -48 & $\begin{array}{r}-43 \\
-3353\end{array}$ & 1 & tgcgtg \\
\hline ENSDARG00000036529 & ENSDARTO0000053081 & Dr.25155.1.S1_s_at & ba2l & Hemoglobin beta-1 chain (BetaA1 globin). [Source:Uniprot/SWISSPROT;ACC:Q990486] & $\begin{array}{l}-3538 \\
-341\end{array}$ & -1336 & -1 & tgcgtg \\
\hline ENSDARG00000036529 & ENSDART00000053081 & Dr.25155.1.S1_s_at & ba2l & Hemoglobin beta-1 chain (BetaA1 globin). [Source:Uniprot/SWISSPROT;ACc:Q90486] & -4476 & -4471 & -1 & tgcgtg \\
\hline ENSDARG00000036531 & ENSDART00000053083 & Dr.1411.1.S1_at & hbaa1 & Hemoglobin alpha chain (Alpha globin aa1). [Source:Uniprot/SWISSPROT;ACc:Q90487] & -2943 & -2938 & 1 & cgcgtg \\
\hline ENSDARG00000036533 & ENSDART00000053086 & Dr.10170.1.A1_at,Dr.10170.1.A1_at & & & -3938 & -3933 & -1 & cgcgtg \\
\hline ENSDARG00000036533 & ENSDART00000053086 & Dr.10170.1.A1_at,Dr.10170.1.A1_at & & & -3823 & -3818 & -1 & tgcgtg \\
\hline ENSDARG00000036533 & ENSDART00000053086 & Dr.10170.1.A1_at,Dr.10170.1.A1_at & & & -3765 & -3760 & 1 & ggcgtg \\
\hline ENSDARG00000036533 & ENSDART00000053086 & Dr.10170.1.A1_at,Dr.10170.1.A1_at & & & -3350 & -3345 & 1 & agcgtg \\
\hline ENSDARG00000036533 & ENSDART00000053086 & Dr.10170.1.A1_at,Dr.10170.1.A1_at & & & -4669 & -4664 & 1 & agcgtg \\
\hline $\begin{array}{l}\text { ENSDARG000000036533 } \\
\text { ENSDARG00000036533 }\end{array}$ & $\begin{array}{l}\text { ENSDART000000053086 } \\
\text { ENSDART0000005086 }\end{array}$ & Dr.10170.1.A1_at,Dr.10170.1.A1_at & & & -4994 & -4989 & -1 & ggcgtg \\
\hline ENSDARG00000036533 & ENSDART00000053087 & $\begin{array}{l}\text { Dr.10170.1.A1_at,D.D.r10170.1.AA_at } \\
\text { Dr.10170.1.A1at,Di.10170.1.A1_at }\end{array}$ & & & $\begin{array}{l}-4931 \\
-3902\end{array}$ & $\begin{array}{l}-3932 \\
-3897\end{array}$ & $\begin{array}{l}-1 \\
-1\end{array}$ & $\begin{array}{l}\text { ggcgrg } \\
\text { cactag }\end{array}$ \\
\hline ENSDARG00000036533 & ENSDART00000053087 & Dr.10170.1.A1_at,Dr.10170.1.A1_at & & & -3787 & -3782 & -1 & tgcgtg \\
\hline ENSDARG00000036533 & ENSDART00000053087 & Dr.10170.1.A1_at,Dr.10170.1.A1_at & & & -3729 & -3724 & 1 & ggcgtg \\
\hline 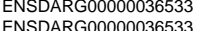 & ENSDART00000053087 & Dr.10170.1.A1_at,Dr.10170.1.A1_at & & & -3314 & -3309 & 1 & agctg \\
\hline $\begin{array}{l}\text { ENSDARG000000036533 } \\
\text { ENSDAG00000036533 }\end{array}$ & $\begin{array}{l}\text { ENSDART000000053387 } \\
\text { ENSDART0000005087 }\end{array}$ & $\begin{array}{l}\text { Dr.10170.1.A1_at,Dr.10170.1.A1_at } \\
\text { DDr10170.A1 at } 101701 \mathrm{A1} \text { at }\end{array}$ & & & -4633 & -4628 & 1 & agcgtg \\
\hline $\begin{array}{l}\text { ENSDARG0000000365533 } \\
\text { ENSDARG0000036533 }\end{array}$ & $\begin{array}{l}\text { ENSDART0000000533087 } \\
\text { ENSDART00000053087 }\end{array}$ & $\begin{array}{l}\text { Dr.10170.1.A1_at,D.D.r10170.1.AA_at } \\
\text { Dr.10170.1.A1 at,D.10170.1.A1 at }\end{array}$ & & & $\begin{array}{l}-4958 \\
-4901\end{array}$ & -48966 & $\begin{array}{l}-1 \\
-1\end{array}$ & $\begin{array}{l}\text { ggcgetg } \\
\text { gactag }\end{array}$ \\
\hline ENSDARG00000036535 & ENSDART00000042396 & $\begin{array}{l}\text { Dr.25155.1.S1_s_at } \\
\text { Dr.1010.1.A1_at }\end{array}$ & ba2 & & -3804 & -3799 & -1 & 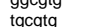 \\
\hline ENSDARG00000036535 & ENSDART00000042396 & Dr.25155.1.S1s_at & ba2 & Hemoglobin beta-1 chain (BetaA1 globin). [Source:Uniprot/SWISSPROT;Acc:Q90486] & -1325 & -1320 & -1 & tgcgtg \\
\hline ENSDARG00000037281 & ENSDART00000054230 & Dr.4907.1.S1_at & NP_998219.1 & fibrinogen gamma polypeptide [Source:RefSeq peptide;ACc:NP 998219 ] & -4801 & -4796 & 1 & cgcgtg \\
\hline ENSDARG00000037618 & ENSDART00000054788 & Dr.13972.1.S1_at & zgc:641144 & hypothetical protein LOC378866 [Source:RefSeq_peptide;ACC:NP_956401] & -3332 & -3327 & 1 & $\begin{array}{l}\text { ggcgtg } \\
\text { gictg }\end{array}$ \\
\hline ENSDARG00000037618 & ENSDART00000054788 & Dr.13972.1.S1_at & zgc:64114 & hypothetical protein LOC378866 [Source:RefSeq_peptide;ACc:NP_956401] & -4700 & -4695 & -1 & cgcgtg \\
\hline ENSDARG00000037618 & ENSDART00000054788 & Dr.13972.1.S1_at & zgc:64114 & & -1223 & -1218 & -1 & tgcgtg \\
\hline ENSDARG00000037618 & ENSDART00000054788 & Dr.13972.1.S1_at & zgc:64114 & hypothetical protein LOC378866 [Source:RefSeq_peptide;Acc:NP_956401] & -1219 & -1214 & -1 & tgcgtg \\
\hline ENSDARG000000037618 & ENSDART00000054788 & Dr.13972.1.S1_at & zgc:64114 & hypothetical protein LOC378866 [Source:RetSeq_peptide;ACc:NP_956401] & -1203 & -1198 & -1 & tgcgtg \\
\hline ENSDARG000000037618 & ENSDART00000054788 & Dr.13972.1.S1_at & zgc:64114 & hypothetical protein LOC378866 [Source:RefSeq_peptide;ACc:NP_956401] & -1199 & -1194 & -1 & tgcgtg \\
\hline $\begin{array}{l}\text { ENSDARG000000037618 } \\
\text { ENSDAG00000037618 }\end{array}$ & ENSDART00000054788 & Dr.13972.1.S1_at & zgc:64114 & hypothetical protein LOC378866 [Source:RefSeq_peptide;Acc:NP_956401] & -578 & -573 & -1 & $\operatorname{tgcgtg}$ \\
\hline $\begin{array}{l}\text { ENSDARG000000037618 } \\
\text { ENSDARG00000037618 }\end{array}$ & $\begin{array}{l}\text { ENSDART000000054788 } \\
\text { ENSDART00000054788 }\end{array}$ & $\begin{array}{l}\text { Dr.13972.1.S1_at } \\
\text { Dr.13972.S1 at }\end{array}$ & $\begin{array}{l}\text { zgc:644114 } \\
\text { zgc:64114 }\end{array}$ & 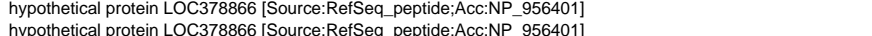 & $\begin{array}{l}-351 \\
-243\end{array}$ & $\begin{array}{l}-346 \\
-238\end{array}$ & $\begin{array}{c}1 \\
-1\end{array}$ & $\begin{array}{l}\text { ggctgtg } \\
\text { ggctg }\end{array}$ \\
\hline ENSDARG00000037618 & $\begin{array}{l}\text { ENSDARTO0000054488 } \\
\text { ENSDART00000054788 }\end{array}$ & $\begin{array}{l}\text { Dr.13992.1.S1_at } \\
\text { Dr.13972.1.S1_at }\end{array}$ & $\begin{array}{l}\text { gac. } 04114 \\
\text { zgc:64114 }\end{array}$ & hypothetical protein LOC378866 [Source:RefSeq_peptide:Acc:NPP 956401] & $\begin{array}{r}-243 \\
-30\end{array}$ & -250 & $\begin{array}{l}-1 \\
-1\end{array}$ & tgcatg \\
\hline ENSDARG00000038321 & ENSDART00000055897 & Dr.9682.1.A1_at & & & -3855 & -3850 & 1 & tgcgtg \\
\hline ENSDARG00000038321 & ENSDART00000055897 & Dr.9682.1.A1_at & & & -1979 & -1974 & -1 & ggcgtg \\
\hline ENSDARG00000038321 & ENSDART00000055897 & Dr.9682.1.A1_at & & & -346 & -341 & -1 & $\begin{array}{l}\text { tgcatg } \\
\text { tgcatg }\end{array}$ \\
\hline ENSDARG00000038321 & ENSDART00000055897 & Dr.9682.1.A1_at & & & -4477 & -4472 & 1 & agcgtg \\
\hline ENSDARG00000038622 & ENSDART00000056382 & Dr.20270.1.S1_at & & & -1704 & -1699 & 1 & agcgtg \\
\hline ENSDARG00000038622 & ENSDART00000056382 & Dr.20270.1.S1_at & & & -4665 & -4660 & 1 & ggcgtg \\
\hline ENSDARG00000038622 & ENSDART00000056382 & Dr.20270.1.S1_at & & & -1420 & -1415 & 1 & ggcgtg \\
\hline 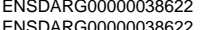 & ENSDART00000056382 & Dr.20270.1.S1_at & & & -1399 & -1394 & -1 & ggcgtg \\
\hline 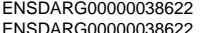 & ENSDART00000056382 & $\begin{array}{l}\text { Dr.20270.1.S1_at } \\
\text { an }\end{array}$ & & & -1221 & -1216 & 1 & $\operatorname{tgcgtg}$ \\
\hline $\begin{array}{l}\text { ENSDARG000000033622 } \\
\text { ENSDARG00000038812 }\end{array}$ & $\begin{array}{l}\text { ENSDART000000056382 } \\
\text { ENSDART00000056679 }\end{array}$ & $\begin{array}{l}\text { Dr.20270.1.S1_at } \\
\text { Dr.20140.A1 at }\end{array}$ & NP 991178.1 & E2F transcription factor 4, p107/p130-binding [Source:RefSeq peptide:Acc:NP 991178] & $\begin{array}{l}-1057 \\
-3847\end{array}$ & $\begin{array}{l}-1052 \\
-3842\end{array}$ & -1 & $\begin{array}{l}\operatorname{tgcgtg} \\
\text { gactag }\end{array}$ \\
\hline & ENSDART00000056680 & $\begin{array}{l}\text { Dr.20140.1.A1_Aa at } \\
\text { Dr.20140. }\end{array}$ & NP 991178.1 & 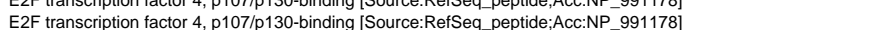 & $\begin{array}{l}-3747 \\
-3732\end{array}$ & 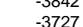 & 1 & ggcgetg \\
\hline ENSDARG00000039027 & ENSDART00000056969 & 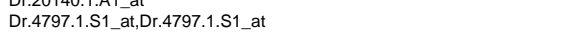 & & & -2978 & -2973 & -1 & agcgtg \\
\hline ENSDARG00000039027 & ENSDART00000056969 & Dr.4797.1.S1_at,Dr.4797.1.S1__at & & & -862 & -857 & -1 & tgcgtg \\
\hline ENSDARG00000039027 & ENSDART00000056969 & Dr.4797.1.S1_at,Dr.4 & & & -540 & -535 & 1 & $\begin{array}{l}\text { gocgtg } \\
\text { gctg }\end{array}$ \\
\hline ENSDARG00000039027 & ENSDART00000056969 & Dr.4797.1.S1_at,Dr.4797.1.S1_at & & & -4068 & -4063 & -1 & agcgtg \\
\hline ENSDARG00000039728 & ENSDART00000037346 & Dr.3581.1.S1_at,Dr.3581.1.S1_a_at & ctrb1 & chymotrypsinogen B1 [Source:RefSeq_peptide;AAcc:NP_997783] & -4839 & -4834 & -1 & ggcgtg \\
\hline ENSDARG00000039747 & ENSDART00000058095 & Dr.4797.1.S1_at & & & -2916 & -2911 & -1 & ggcgtg \\
\hline ENSDARG00000039747 & ENSDART00000058095 & Dr.4797.1.S1_at & & & -2797 & -2792 & 1 & agcgtg \\
\hline ENSDARG00000039747 & ENSDART00000058095 & Dr.4797.1.S1_at & & & -2399 & -2394 & 1 & agcgtg \\
\hline $\begin{array}{l}\text { ENSDARG0000000039747 } \\
\text { ENSDARG0000039841 }\end{array}$ & $\begin{array}{l}\text { INSDART000000050895 } \\
\text { ENSDART00000058272 }\end{array}$ & $\begin{array}{l}\text { D.r.7997.1.S1_at } \\
\text { D.7110.1.S1 at }\end{array}$ & zgc: 77614 & hypothetical protein LOC393868 [Source:RefSeq_peptide;ACc:NP_957188] & $\begin{array}{l}-1967 \\
-2848\end{array}$ & $\begin{array}{l}-1962 \\
-2843\end{array}$ & $\begin{array}{c}-1 \\
1\end{array}$ & $\begin{array}{l}\text { tgcgtg } \\
\text { tgcgtg }\end{array}$ \\
\hline ENSDARG000000039914 & ENSDART00000058384 & $\begin{array}{l}\text { AFFX-Dr-GAPDDH-3_at,AFFX-Dr-GAPDH-M_at,Dr.1194.1.S1_at,AFFX-Dr- } \\
\text { GAPDH-5 at }\end{array}$ & zgc:76908 & glyceraldehyde 3-phosphate dehydrogenase [Source:RefSeq_peptide;Acc:NP_998259] & -3815 & -3810 & 1 & agcata \\
\hline & & AFFX-Dr-GAPDH-3_at,AFFX-Dr-GAPDH-M_at,Dr.1194.1.S1_at,AFFX-Dr- & & glyceraldehyde 3-phosphate dehydrogenase [Source-RefSeq peptide:Acc-NP 998259] & & & & aglegly \\
\hline ENSDARG00000039914 & ENSDART00000058384 & $\begin{array}{l}\text { GAPDH-5_at } \\
\text { AFFX-Dr-GAPDH-3 at.AFFX-Dr-GAPDH-M at,Dr.1194.1 }\end{array}$ & zgc:76908 & glyceraladenyde 3-phosphate denyarogenase [Source:Rerseq_peptide;ACc:NP__98259] & -2730 & -2725 & 1 & agcgtg \\
\hline ENSDARG00000039914 & ENSDART00000058384 & GAPDH-5_at & zgc:76908 & glyceraldehyde 3-phosphate dehydrogenase [Source:RefSeq_peptide;Acc:NP_998259] & -1064 & -1059 & 1 & $\operatorname{tgcgtg}$ \\
\hline ENSDARG00000039914 & ENSDART00000058384 & $\begin{array}{l}\text { AFFX-Dr-GAPDH-3_at,_AFFX-Dr-GAPDH-M_at,Dr.1194.1.S1_at,AFFX-Dr- } \\
\text { GAPDH-5_at }\end{array}$ & zgc:76908 & glyceraldehyde 3-phosphate dehydrogenase [Source:RefSeq_peptide;Acc:NP_998259] & -3815 & -3810 & 1 & agcgtg \\
\hline ENSDARG00000039914 & ENSDART00000058384 & 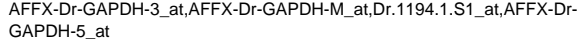 & zgc:76908 & glyceraldehyde 3-phosphate dehydrogenase [Source:RefSeq_peptide;Acc:NP_998259] & -2730 & -2725 & 1 & agcgtg \\
\hline 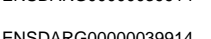 & & AFFX-Dr-GAPDH-3_a & & glyceraldehyde 3-phosphate dehydrogenase [Source:RefSeq peeptide;Acc:NP 998259] & & & & \\
\hline ENSDARG00000039914 & ENSDART00000058384 & GAPDH-5_at & zgc:76908 & & -1064 & -1059 & 1 & $\operatorname{tgcgtg}$ \\
\hline ENSDARG00000039914 & ENSDART00000058384 & 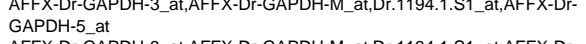 & zgc:76908 & glyceraldehyde 3-phosphate dehydrogenase [Source:RefSeq_peptide;Acc:NP_998259] & -3815 & -3810 & 1 & agcgtg \\
\hline ENSDARG00000039914 & ENSDART00000058384 & $\begin{array}{l}\text { AFFX-Dr-GAPDH-3_at,AFFX-Dr-GAPDH-M_at,Dr.1194.1.S1_at,AFFX-Dr- } \\
\text { GAPDH-5_at }\end{array}$ & zgc:76908 & glyceraldehyde 3-phosphate dehydrogenase [Source:RefSeq_peptide;Acc:NP_998259] & -2730 & -2725 & 1 & agcgtg \\
\hline ENSDARG0000003914 & ENSDART00000058384 & $\begin{array}{l}\text { AFFX-Dr-GAPDH-3_at,AFFX-Dr-GAPDH-M_at,Dr.1194.1.S1_at,AFFX-Dr- } \\
\text { GAPDH-5 at }\end{array}$ & zgc:76908 & glyceraldehyde 3-phosphate dehydrogenase [Source:RefSeq_peptide;Acc:NP_998259] & -1064 & -1059 & 1 & tgcgtg \\
\hline ENSDARG00000039914 & ENSDART00000058384 & $\begin{array}{l}\text { AFFX-Dr-G̈APDH-3_at,AFFX-Dr-GAPDH-M_at,Dr.1194.1.S1_at,AFFX-Dr- } \\
\text { GAPDD-5 at at }\end{array}$ & 908 & glyceraldehyde 3-phosphate dehydrogenase [Source:RefSeq_peptide;Acc:NP_998259] & -3815 & -3810 & + & (9) \\
\hline & & AFFX-Dr-GAPDH-3_at,AFFX-Dr-GAPDH-M_at,Dr.1194.1.S1_at,AFFX-Dr- & 20.10000 & glyceraldehyde 3-phosphate dehydrogenase [Source:RefSeq_peptide;Acc:NP 998259$]$ & 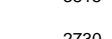 & (2010 & & ( \\
\hline ENSDARG000000039914 & ENSDART00000058384 & GAPDH-5_at & zgc:76908 & & -2730 & -2725 & 1 & agcgtg \\
\hline
\end{tabular}



作X-Dr-GAPDH-3_at,AFFX-Dr-GAPDH-M_at,Dr.1194.1.S1_at,AFFX-DrGAPDH-5_at ENSARTO00000

ENSDARG00000041384

ENSDARG00000041384

ENSDARG00000041384

ENSDARG00000041384

ENSDARG00000041384

ENSDARG00000041384

ENSDARG00000041384 ENSDARG00000041633 NSDARG00000041633 ENSDARG00000041633
ENSDARG00000041633

ENSDARG00000041639 ENSDARG00000041639

ENSDARG00000041639 ENSDARG00000041639 ENSDARG00000041639 ENSDARG00000041639 ENSDARG00000041639 ENSDARG00000041639 ENSDARG00000041639 ENSDARG00000041639 ENSDARG00000041639 ENSDARG00000041639 ENSDARG00000041645 ENSDARG00000041645 ENSDARG00000041653 ENSDARG00000041685 ENSDARG00000041685 ENSDARG00000041685 ENSDARG00000041685 ENSDARG00000041685 FFX-Dr-GAPDH-3_at,AFFX-Dr-GAPDH-M_at,Dr.1194.1.S1_at,AFFX-DrAFFX-Dr-GAPDH-3_at,AFFX-Dr-GAPDH-M_at,Dr.1194.1.S1_at,_AFFX-D

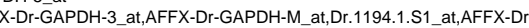
zgc:76908 ENSDART00000060668 ENSDART00000060668 ENSDART00000060668 ENSDART0000006066 ENSDART00000060668 ENSDARTT0000041093 ENSDARTO0000041093 ENSDARTO0000041093 ENSDART00000044076 ENSDART00000044076 ENSDART00000046454 ENSDART00000046454 ENSDART00000047098 ENSDART00000047098 ENSDART0000004407 ENSDART0000004407 ENSDART00000046454 ENSDART0000004645 ENSDART00000047098 ENSDART00000047098 ENSDART00000046443 ENSDARTO0000046443 ENSDART00000061046 ENSDART0000006104 ENSDART00000006984 ENSDART00000006984 ENSDART00000006984 ENSDART00000061093 ENSDART00000061093

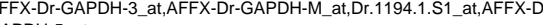
AFFX-Dr-GAPDH-3 at,AFFX-Dr-GAPDH-M_at,Dr.1194.1.S1_at,AFFX-DrAFFX-Dr-GAPDH-3_at,AFFX-Dr-GAPDH-M_at,Dr.1194.1.S1_at,_AFFX-DrGAPDH-5_-at GAPDH-5.at at
Dr.23812.1.A1_at,Dr.25379.1.S1_at,Dr.3025.3.S1_at
Dr.23812.A1_atDr.25379.1S1_at_Dr.30253.S1_at Dr.23812.1.A1_at,Dr.25379.1.S1_at,Dr.3025.3.S1_a Dr.238121.A1_at,D.25379.1.S1_at,Dr.3025.3.S1_. Dr.23812.1.A1_at,Dr.25379.1.S1_at,Dr.3025.3.S1_at Dr.23812.1.A1_at,Dr.25379.1.S1_at,Dr.3025.3.S1 Dr.25379.1.S1_at,Dr.23812.1.A1_at,Dr.25379.1.S1_at,Dr.3025.3.S1_at,Dr.238 Dr.25379.1.S1 at.Dr.23812.1.A1 at.Dr.25379.1 2.1.A1_at,Dr.25379.1.S1_at,Dr.3025.3.S1_at $a 1$ _. at,Dr.3025.3.S1_at,Dr.238 Dr.25379.1.S1_at,Dr.23812.1.A1_at,Dr.25379.1.S1_at,Dr.3025.3.S1_at,Dr.238 Dr.25379.1.S1 at,Dr.23812.1.A1 at,Dr.25379.1.S1_at,Dr.3025.3.S1_at,Dr.238

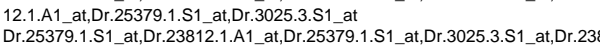
Dr.25379.1.S1_at,Dr.23812.1.A1_at,Dr.25379.1.S1_at,Dr.3025.3.S1_at,Dr.23
12.1.A1_at,Dr.25379.1.S1_at,Dr.3025.3.S1_at Dr.25379.1.S1_at,Dr.23812.1.A1_at,Dr.25379.1.S1_at,Dr.3025.3.S1_at,Dr.238 12.1.A1_at,Dr.25379.1.S1_at,Dr.3025.3.31_at _.251_at.Dr.3025.3.S1_at,Dr.238

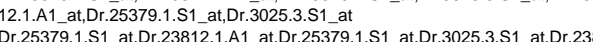
12.1.A1_at,Dr.25379.1.S1_at,Dr.3025.3.S1_at at_at,Dr.3025.S1_at,Dr.238
Dr.25379.1.S1_at,Dr.23812.1.A1_at,Dr.25379.1.S1_at,Dr.3025.3.S1_at,Dr.238 12.1.A1_at,Dr.25379.1.S1_at,Dr.3025.3.S1_at

at,Dr.3025.3.S1_at,Dr.238 12.1.A1_at,Dr.25379.1.S1_at,Dr.3025.3.S1_at 01 _.

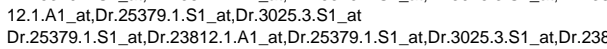
12.1.A1_at,Dr.25379.1.S1_at,Dr.3025.3.S1_at Dr.25379.1.S1_at,Dr.3025_.2.S1_at,Dr.3025.3.S1_at

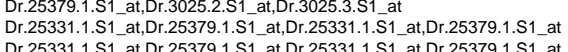
Dr.25331.1.S1_at,Dr.25379.1.S1_at,Dr.25331.1.S1_at,Dr.25379.1.S1_at Dr.25379.1.S1_at,Dr.3025.3.S1_at,Dr.16397.1.A1_at,Dr.25379.1.S1_at,Dr.302

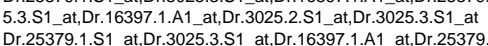

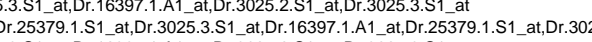
5.3.S1_at,Dr.16397.1.A1 at,Dr.3025.2.S1_at,Dr.3025.3.S1_at Dr.25379.1.S1_at,Dr.3025.3.S1_at,Dr.26397.1.A1_at,Dr.25379.1.S1_at,Dr.302

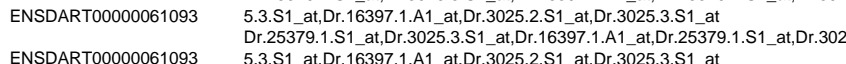

zgc: 76908

NP_-999949.1

NP 999994919
zgc: 92317
zgc:92317

apoes

apoeb

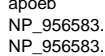

NP_956583.1

zgc: 77059

Idha

Idha
Idha
Idha

Tosphate dehydrogenase [Source:RefSeq_peptide;Acc:NP_998259]

actin, alpha, cardiac muscle [Source:RefSeq_peptidd;AACc:NP_999949
actin, alpha, cardiac muscle [Source:RefSeq peptide:Acc:NP-999949

actin, alpha, cardiac muscle [Source:RefSeq_peptide;AAcc:NP_999949]
hypothetical protein LOC449545 [Source:RefSeq peptide:Acc:NP 001005587

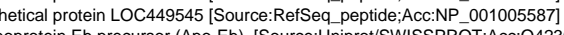
Apolipoprotein Eb precursor (Apo-Eb). [Source:UniprotsSWISSPROT:Acc:042364]
Apolipoprotein Eb precursor (Apo-Eb). [Source:UniprotSSWISSPROT;ACc:O42364] Apolipoprotein Eb precursor (Apo-Eb). [Source:UniprotsWISSPROT:AAc:O42364
Apolipoprotein Eb precursor (Apo-Eb). [Source:UniprotsWWISSPROT:Acc:O42364 similar to yolk sac gene 2 [Source:RefSeq_peptide;Acc:NP_956583]
similar to yolk sac gene 2 [Source:RefSeq_peptide;Acc:NP_-956583] similar to yolk sac gene 2 [Source:RefSeq_peptide;Acc:NP_-956583 similar to yolk sac gene 2 [Source:RefSeq_peptide;Acc:NP_-956583]
hypothetical protein LOC405809 [Source:RefSeq_peptide;Acc:NP_998039 hypothetical protein LOC405809 [Source:RefSeq_peptide;ACc:NP_998038]

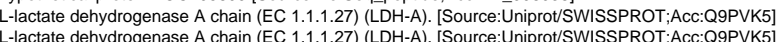
-lactate dehydrogenase $A$ chain (EC 1.1.1.27) (LDH-A). [Source:Uniprot/SWISSPROT;ACc:Q9PVK5

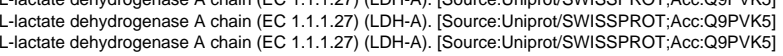
glycraldehyde 3-phosphate dehydrogenase [Source:RefSeq_peptide;Acc:NP_998259] glyceraldehyde 3-phosphate dehydrogenase [Source:RefSeq_peptide;Acc:NP_998259] glyceraldehyde 3-phosphate dehydrogenase [Source:RefSeq_peptide;Acc:NP_998259] glyceraldehyde 3-phosphate dehydrogenase [Source:RefSeq_peptide;Acc:NP_998259] glyceraldehyde 3-phosphate dehydrogenase [Source:RefSeq_peptide;Acc:NP_998259] glyceraldehyde 3-phosphate dehydrogenase [Source:RefSeq_peptide;Acc:NP_998259] glyceraldehyde 3-phosphate dehydrogenase [Source:RefSeq_peptide;Acc:NP_998259] glyceraldehyde 3-phosphate dehydrogenase [Source:RefSeq_peptide;Acc:NP_998259] glyceraldehyde 3-phosphate dehydrogenase [Source:RefSeq_peptide;Acc:NP_998259] glyceraldehyde 3-phosphate dehydrogenase [Source:RefSeq_peptide;Acc:NP_998259] glyceraldehyde 3-phosphate dehydrogenase [Source:RefSeq_peptide;Acc:NP_998259] glyceraldehyde 3-phosphate dehydrogenase [Source:RefSeq_peptide;ACc:NP_998259] 
ENSDARG00000041685 ENSDARG00000041685 ENSDARG00000041685 ENSDARG00000041685 ENSDARG00000041685 ENSDARG00000041685 ENSDARG00000041685 ENSDARG00000041685 ENSDARG00000041685 ENSDARG00000041685 ENSDARG00000041685 ENSDARG00000041685
ENSDARG00000041769 ENSDARG00000041769 ENSDARG00000041774 ENSDARG00000041813 ENSDARG00000041813 ENSDARG00000041997 NSDARG00000041997 ENSDARG000000041997 ENSDARG000000041997 ENSDARG00000041997 ENSDARG00000041997 ENSDARG00000041997 ENSDARG00000041997 ENSDARG000000042035 ENSDARG000000042036 ENSDARG000000042036 NSDARG00000042036 ENSDARG00000042040 NSDARG00000042040 NSDARG00000042040 ENSDARG00000042040 ENSDARG00000042040 ENSDARG000000042046

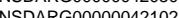
ENSDARG00000042391 ENSDARG000000042391 ENSDARG000000043218 ENSDARG00000043218 ENSDARG00000043320 ENSDARG000000043320 ENSDARG00000043592 ENSDARG00000043592 ENSDARG00000043592 ENSDARG00000043592 ENSDARG00000043592 ENSDARG00000043592 ENSDARG00000043618

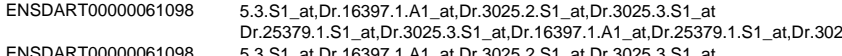

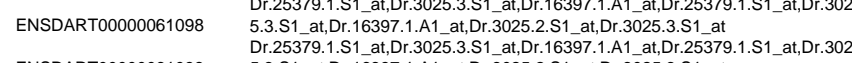
ENSDART00000061098 ENSDART00000006984 ENSDART00000006984 ENSDART00000006984 ENSDART00000061093 ENSDART00000061093 ENSDART00000061093 ENSDART00000061098 ENSDART00000061098 ENSDART00000061098 ENSDARTO0000061219 ENSDART0000006122 ENSDART00000061223 ENSDART00000061223 ENSDART000000061279 ENSDARTO000006127 ENSDARTT00000061544 ENSDARTO0000006154 ENSDARTT0000006154 ENSDARTO0000006154 ENSDART0000006154 ENSDARTO0000006154 ENSDART0000006154 ENSDART00000061544 ENSDARTO0000061544 ENSDARTO0000006154 ENSDART00000061544 ENSDARTO000006160 ENSDART00000061605 ENSDARTO0000061605 ENSDARTO00000061615 ENSDARTO0000061605 ENSDARTO0000061613 ENSDARTO0000061613 ENSDART00000061613 ENSDART00000061613 ENSDARTO0000061613 ENSDARTO0000061618 ENSDART00000061623 ENSDARTO000006170 ENSDARTO0000006215 ENSDARTO000006345 ENSDART0000006345 ENSDART00000063453 ENSDART0000006359 ENSDARTO0000006359 ENSDART00000064008 ENSDART00000064008 ENSDART00000064008 ENSDART00000064010 ENSDART00000064010 ENSDART00000064010 ENSDART0000006404

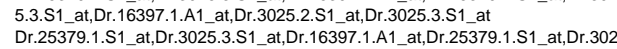

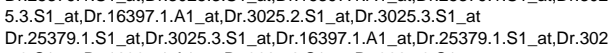

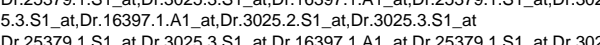
5.3.S1_at,Dr.16397.1.A1_at,Dr.3025.2.2S1_at,Dr._3025.3.3.S1_at at.S1_at,Dr.302 Dr.25379.1.S1_at,DPr.3025.3.S1_at,Dr.16397.1.A1_at,Dr.25379.1.S1_at,Dr.302

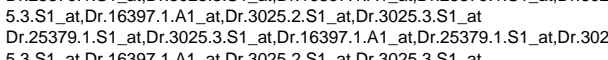

5.3.S1_at,Dr.16397.1.A1_at,Dr.3025.2.S1_at,DPr.3025.3.S1_at 1 at.25379.1.S1 at.Dr.3025.3.S1 at.Dr.16397.1.A1 at.Dr.25379.1.S1_at,Dr.302

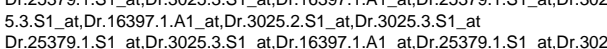
Dr.25379.1.S1_at,Dr.3025.3.S1_att,Dr.16397.1.A1_at,Dr.25379.1.S1_at,Dr.302
5.3.31_at,Dr.16397.1.A1_at,Dr.3025.2.S1_at,Dr.3025.3.S1_at

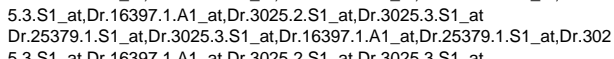
Dr.7806.1.A1_at
Dr.7806.1.A1_at
Dr.7806.1.A1 at

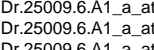
Dr.25009.6.A1_a_a Dr.7806.1.A1_at Dr.7806.1.A1_at Dr.12106.1.S1_at Dr.12106.1.S1_at Dr.12106.1.S1_at Dr.12106.1.S1_at Dr.12106.1.S1_at Dr.12106.1.S1_at Dr.12106.1.S1_at Dr.12106.1.S1_at Dr.12106.1.S1 at Dr.12106.1.S1_at Dr.12106.1.S1_at Dr.12106.1.S1_at
Dr.12106.1.S1_at Dr.12106.1.S1_at Dr.7110.1.S1_at
Dr.7110.1S1 at Dr.7110.1.s1_a D.7110.1.S1_at Dr.7110.1.S1_at

Dr.7110.1.S1_at
Dr.7110.1.S1 at

Dr.7110.1.S1_at
Dr.7110.1.S1_at

Dr.7110.1.S1 at

Dr.7110.1.S1_a

Dr.7110.1.s1_at

Dr.7110.1.S1_at

Dr.7110.1.S1_at
Dr.7110.1.S1_at

Dr.7110.1.S1_at
Dr.7110.1.S1_at

Dr.7110.1.S1_at

Dr.14748.1.S1_at

Dr.14748.1.S1_at

Dr.15054.1.S1_at
Dr.15054.1.S1_at

Dr.15054.1.s1_at
Dr.15054.1.S1_at

Dr.14268.1.A1-at,Dr.18893.1.S1-at
Dr.14268.1.A1_at,Dr.18893.1.S1_at Dr.7236.1.A1_at,Dr.7236.1.A1_at
Dr.14268.1.AA_at,Dr.18893.1.S1_a

Dr.17113.1.A1_a_at,Dr.3889.1.A1_at,Dr.17113.1.A1_a_at,Dr.3889.1.A1_at ptena phosphatase and tensin-like protein A [Source:RefSeq_peptide,Acc:NP_957002] Dr.17113.1.A1_a_at,Dr.3889.1.A1_at,Dr.17113.1.A1_a_at,Dr.3889.1.A1_at ptena Dr.17113.1.A1_a_at,Dr.3889.1.A1_at,Dr.17113.1.A1_a_at,Dr.3889.1.A1_at ptena Dr.17113.1.A1_a_at,Dr.3889.1.A1_at,Dr.17113.1.A1_a_at,Dr.3889.1.A1_at Dr.17113.1.A1_a_at,Dr.3889.1.A1_at,Dr.17113.1.A1_a_at,Dr.3889.1.A1_at ptena Dr.17113.1.A1_a_at,Dr.3889.1.A1_at,Dr.17113.1.A1_aat,Dr.3889.1.A1_at ptena
NP_001014360.1

NP-001014360.1

ypothetical protein LOC541525 [Source:RefSeq_peptide;Acc:NP_001014360]

NP_001014360.1 hypothetical protein LOC541525 [Source:RefSeq_peptide;Acc:NP_001014360

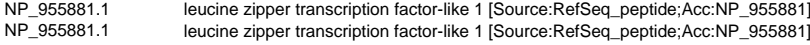

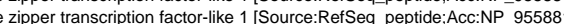
NP_9558811

NP_955881.1

NP_-955881.

NP-955881.

NP_-955881.1.

NP_955881.

NP_955881.

zgc: 77614

zgc: 77614

zgc.:77614
zgc: $: 77614$
zgc: 77614

zgc:77614

zgc: 77614

zgc: 77614

zgc:63985

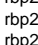

-

hypothetical protein LOC393868 [Source:RefSeq_peptide;ACc:NP_957188] hypothetical protein LOC3933868 [Source:RefSeq_peptide;AAcc:NP_-957188] W (protein for MGC:66449) [Source:RefSeq_peptide;Acc:NP_956095]

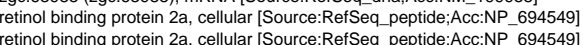
retinol binding protein 2a, cellular [Source:RefSeq_peptide:Acc:NP_694549]
retinol binding protein 2a, cellular [Source:RefSeq_peptide:Acc:NP_694549] phosphatase and tensin-like protein A [Source:RefSeq_peptide;ACc:NP_957002] phosphatase and tensin-like protein A [Source:RefSeq_peptide;Acc:NP_957002] phosphatase and tensin-like protein A [Source:RefSeq_peptide;Acc:NP_957002] phosphatase and tensin-like protein A [Source:RefSeq_peptide;Acc:NP_957002] phosphatase and tensin-like protein A [Source:RefSeq_peptide;Acc:NP_957002]

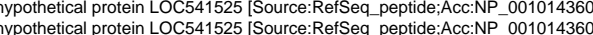
NP_001014360.1 hypothetical protein LOC541525 [Source:RefSeq_Peptide;ACc:NP_001014360 zgc:63985 (zgc:63985), mRNA [Source:RefSeq_dna;AAcc:NM_199958]
zgc:63985 (zgc:63985), mRNA [Source:RefSea_dna:Acc:NM_199958 


\begin{tabular}{|c|c|c|c|c|c|c|c|c|}
\hline sene ID & Iranscript id & IDs & Symbol & & Start & End & Strand & Sequence \\
\hline ENSDARG0 & $\overline{00064041}$ & Dr.7236.1.A1_at,Dr.7236.1.A1_at & & & $\overline{-2732}$ & & e & cegcgtg \\
\hline $\begin{array}{l}\text { ENSDARG000000043618 } \\
\text { ENSDARG00000043618 }\end{array}$ & ENSDARTO0000064044 & Dr.7236.1.A1_at,Dr.7236.1.A1_at & & & -2734 & -2 & -1 & cgcgtg \\
\hline ENSDARG00000043618 & & Dr.7236.1.A1_at,Dr.7236.1.A1_at & & serine (or cysteine) proteinase inhibitor, clade A (alpha-1 antiproteinase, antitypsin), member 1 (serpina1), mRNA & -2732 & -2727 & 1 & cgcgtg \\
\hline ENSDARG00000043670 & ENSDART00000024462 & Dr.1605.1.S1_at & NM_001013259.1 & [Source:RefSeq_dna;ACc:NM_001013259] & -4819 & -4814 & 1 & agcgtg \\
\hline ENSDARG00000043670 & & Dr.1605.1.S1_at & NM 0010132591 & $\begin{array}{l}\text { serine (or cysteine) proteinase inhibitor, clade A (alpha-1 antiproteinase, antitypsin), member } \\
\text { STource- }\end{array}$ & & & & \\
\hline ENSDARG00000043692 & ENSDART00000064157 & Dr.19877.1.S1 at & zgc:66133 & $\begin{array}{l}\text { hypothetical protein LOC } 10393468 \text { [Source:RefSeg pepti } \\
\text { hypons }\end{array}$ & $\begin{array}{l}-4071 \\
-3071\end{array}$ & $\begin{array}{l}-4100 \\
-3066\end{array}$ & $\begin{array}{c}1 \\
-1\end{array}$ & $\begin{array}{l}\text { agcgig } \\
\text { tgcatg }\end{array}$ \\
\hline ENSDARG00000043692 & ENSDART00000064157 & Dr.19877.1.S1_at & zgc:66133 & hypothetical protein LOC393468 [Source:RefSeq_peptide;ACC:NP 956790$]$ & -2789 & -2784 & 1 & cgcgtg \\
\hline ENSDARG00000043692 & ENSDART00000064157 & Dr.19877.1.S1_at & zgc:66133 & hypothetical protein LOC393468 [Source:RefSeq_peptide;ACc:NP_-956790] & -2454 & -2449 & -1 & ggcgtg \\
\hline ENSDARG00000043692 & ENSDARTO00000064157 & Dr.19877.1.S1_at & zgc:66133 & hypothetical protein LOC393468 [Source:RefSeq_peptide;ACc:NP_-956790] & -1360 & -1355 & 1 & ggcgtg \\
\hline ENSDARG00000043692 & ENSDART00000064157 & Dr.19877.1.S1_at & zgc:66133 & hypothetical protein LOC393468 [Source:RefSeq_peptide;ACC:NP_956790] & -942 & -937 & -1 & cgcgtg \\
\hline ENSDARG000000043692 & ENSDART00000064157 & Dr.19877.1.S1_at & zgc:66133 & hypothetical protein LOC393468 [Source:RefSeq_peptide;ACc:NP_956790] & -105 & -100 & -1 & cgcgtg \\
\hline $\begin{array}{l}\text { ENSDARG000000043692 } \\
\text { ENDARG00000043960 }\end{array}$ & & Dr.19877.1.S1_at & zgc:66133 & hypothetical protein LOC393468 [Source:RefSeq_peptide;Acc:NP_956790] & -16 & -11 & -1 & cgcgtg \\
\hline $\begin{array}{l}\text { ENSDARG000000003960 } \\
\text { ENSDARG00000043960 }\end{array}$ & $\begin{array}{l}\text { ENSDARTO00000064542 } \\
\text { ENST }\end{array}$ & Dr.6147.1.A1_at & zgc:77150 & hypothetical protein LOC406587 [Source:RefSeq_peptide;Acc:NP_9999913] & -3939 & -3934 & -1 & agcgtg \\
\hline $\begin{array}{l}\text { ENDSARG0000000033960 } \\
\text { ENSDARG00000043960 }\end{array}$ & $\begin{array}{l}\text { ENSDARTO00000006542 } \\
\text { ENSDATT0000064542 }\end{array}$ & $\begin{array}{l}\text { Dr.61477.1.A1_at } \\
\text { Dr.6147.1.A1 at }\end{array}$ & $\begin{array}{l}\text { zgc:777150 } \\
\text { zac:77150 }\end{array}$ & 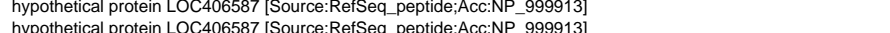 & $\begin{array}{l}-4689 \\
-552\end{array}$ & -4684 & 1 & cgcgtg \\
\hline ENSDARG00000043960 & ENSDART00000064542 & $\begin{array}{l}\text { Dr.6147.1.A1_at at } \\
\text { Dr. Al }\end{array}$ & $\begin{array}{l}\text { gaci.71750 } \\
\text { zgc:77150 }\end{array}$ & 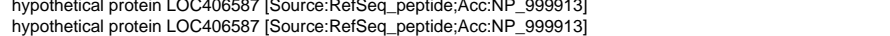 & $\begin{array}{l}-452 \\
-4539\end{array}$ & $\begin{array}{l}-5534 \\
-454\end{array}$ & $\begin{array}{l}1 \\
1\end{array}$ & $\begin{array}{l}\operatorname{lgctg} \\
\operatorname{tgctg}\end{array}$ \\
\hline ENSDARG00000043990 & ENSDART00000064579 & Dr.7806.1.A1_at & & & -138 & -133 & & tgcgtg \\
\hline ENSDARG00000043997 & ENSDART00000064586 & Dr.11609.1.S1_at & Q7SZD0 BRARE & LOC402847 protein (Fragment). [Source:Uniprot/SPTREMBL:ACC:07SZDO] & -2929 - & -2924 & 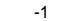 & aacotg \\
\hline ENSDARG00000043997 & ENSDART00000064586 & Dr.11609.1.S1_at & Q7SZDO_BRARE & LOC402847 protein (Fragment). [Source:Uniprot/SPTREMBL:ACC:Q7SZDO] & $=600$ & -595 & -1 - & $\begin{array}{l}\text { tocgtg } \\
\text { tcotg }\end{array}$ \\
\hline ENSDARG00000043997 & ENSDART00000064586 & Dr.11609.1.S1_at & Q7SZDO_BRARE & LOC402847 protein (Fragment). [Source:Uniprot/SPTREMBL:ACC:Q7SZDO] & -552 & -547 & -1 & ${ }_{\text {tgcotg }}$ \\
\hline ENSDARG00000043997 & ENSDART00000064586 & Dr.11609.1.S1_at & Q7SZDO_BRARE & LOC402847 protein (Fragment). [Source:Uniprot/SPTREMBL;ACC:Q7SZDO] & -67 & -62 & 1 & ggcgtg \\
\hline ENSDARG00000044001 & ENSDART00000064597 & Dr.20815.3.A1_at & NP_999858.1 & chimera galectin Gal3 [Source:RefSeq_peptide;Acc:NP_999858] & -3720 & -3715 & -1 & tgcgtg \\
\hline ENSDARG000000044001 & ENSDART00000064597 & Dr.20815.3.A1_at & NP_999858.1 & chimera galectin Gal3 [Source:RefSeq_peptide;Acc:NP_-999858] & -371 & -366 & -1 & tgcgtg \\
\hline ENSDARG000000044002 & ENSDART00000064591 & Dr.11609.1.S1_at,Dr.11609.1.S1_at & NP_001002187.1 & hypothetical protein LOC431734 [Source:RefSeq_peptide;Acc:NP_001002187] & -3995 & -3990 & -1 & tgcgtg \\
\hline $\begin{array}{l}\text { ENSDARG000000044002 } \\
\text { ENDARG000004402 }\end{array}$ & $\begin{array}{l}\text { ENSDARTO00000064591 } \\
\text { ENSDART0000004591 }\end{array}$ & Dr.11609.1.S1_at,Dr.11609.1.S1_at & NP_001002187.1 & & -4812 & -4807 & -1 & agcgtg \\
\hline $\begin{array}{l}\text { ENDSARG0000000044002 } \\
\text { ENSDARG00000044002 }\end{array}$ & $\begin{array}{l}\text { ENSDARTO000000064591 } \\
\text { ENSDART0000064591 }\end{array}$ & $\begin{array}{l}\text { Dr.11609.1.S1__at,Dr.116099.1.S1_at } \\
\text { Dr.11609.1S1 at.Dr.1160.1.S1 at }\end{array}$ & $\begin{array}{l}\text { NP_-001002187.1 } \\
\text { NP } 001002187.1\end{array}$ & 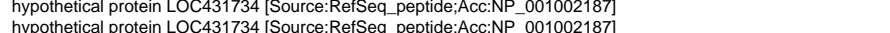 & -4517 & $\begin{array}{l}-4967 \\
-1512\end{array}$ & ${ }_{1}^{-1}$ & $\begin{array}{l}\operatorname{lgctg} \\
\text { tgctgatg }\end{array}$ \\
\hline ENSDARG000000044002 & ENSDART00000064591 & $\begin{array}{l}\text { Dr.116099.1.S1_at,Dr.116099.1.S1_at } \\
\text { Dr.11609.1. at,Dr.11609.S1_at }\end{array}$ & NP 001002187.1 & 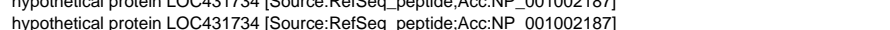 & $\begin{array}{l}-111 / \\
-439\end{array}$ & $\begin{array}{l}-1512 \\
-434\end{array}$ & $\begin{array}{c}1 \\
-1\end{array}$ & $\begin{array}{l}r g c c 19 \\
\text { tgcotg }\end{array}$ \\
\hline ENSDARG00000044002 & ENSDART00000064596 & Dr.11609.1.S1 at,Dr.11609.1.S1_at & NP-001002187.1 & hypothetical protein LOC431734 [Source:RefSeq peptide:Acc:NP 001002187 ] & $\begin{array}{l}-4653 \\
-1653\end{array}$ & $\begin{array}{c}-4648 \\
-1648\end{array}$ & $\begin{array}{l}-1 \\
-1\end{array}$ & ggcotg \\
\hline ENSDARG00000044002 & ENSDART00000064596 & Dr.11609.1.S1 at,Dr.11609.1.S1 at & NP 001002187.1 & 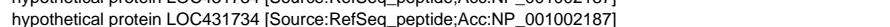 & -1300 - & -1295 & -1 & 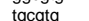 \\
\hline ENSDARG00000044002 & ENSDART00000064596 & Dr.11609.1.S1_at,Dr.11609.1.S1_at & NP_001002187.1 & hypothetical protein LOC431734 [Source:RefSeq_peptide;ACc:NP_001002187] & -1140 & -1135 & -1 & agcgtg \\
\hline ENSDARG00000044002 & ENSDART00000064596 & Dr.11609.1.S1_at,Dr.11609.1.S1_at & NP- 001002187.1 & hypothetical protein LOC431734 [Source:RefSeq_peptide;ACc:NP_001002187] & -323 & -318 & -1 & tgcotg \\
\hline ENSDARG00000044101 & ENSDART00000064753 & Dr.18135.1.A1_at & zgc: 92568 & zgc:92568 [Source:RefSeq_peptide;Acc:NP_001005937] & -4881 & -4876 & 1 & ggcgtg \\
\hline ENSDARG00000044101 & ENSDART00000064753 & Dr.18135.1.A1_at & zgc:92568 & zgc:92568 [Source:RefSeq_peptide;Acc:NP_001005937] & -475 & -470 & 1 & agcgtg \\
\hline ENSDARG00000044125 & ENSDART00000064789 & Dr.8723.1.S1_at & zgc:92903 & zgc:92903 [Source:RefSeq_peptide;Acc:NP_001002461] & -1108 & -1103 & -1 & agcgtg \\
\hline ENSDARG000000044125 & ENSDART00000064789 & Dr.8723.1.S1_at & zgc:92903 & zgc:92903 [Source:RefSeq_peptide;ACc:NP_001002461] & -48 & -43 & 1 & tgctg \\
\hline ENSDARG000000044176 & ENSDART00000064858 & Dr.23470.1.S1_s_at,Dr.8198.1.A1_at & $\mathrm{krml2.2}$ & Kreisler (mouse) maf-related leucine zipper homolog 2.2 [Source:RefSeq_peptide;Acc:NP_571917] & -3256 & -3251 & -1 & agcgtg \\
\hline $\begin{array}{l}\text { ENSDARG0000000441776 } \\
\text { ENDARG000004476 }\end{array}$ & $\begin{array}{l}\text { ENSDARTT00000064858 } \\
\text { ENSDATO00000064858 }\end{array}$ & $\begin{array}{l}\text { Dr.23470.1.S1_s_at,Dr.8198.1.A1_at } \\
\text { Dr.23470. S1 s atDr at }\end{array}$ & $\begin{array}{ll}\mathrm{kmm} / 2.2 \\
\mathrm{~km} / 2.2\end{array}$ & Kreister (mouse) maf-related leucine zipper homolog 2.2 [Source:RefSeq_peptide;Acc:NP_571917] & -1753 & -1748 & -1 & tgcgtg \\
\hline $\begin{array}{l}\text { ENSDARG00000000441176 } \\
\text { ENSDARG0000044176 }\end{array}$ & $\begin{array}{l}\text { ENSDARTO000000048858 } \\
\text { ENSDART0000064858 }\end{array}$ & $\begin{array}{l}\text { D.r.23477.1.S1_s__at,Dr.8198.1.A1_at } \\
\text { D.23470.1.S1 s at.Dr.8198.A1 at }\end{array}$ & $\begin{array}{l}\mathrm{kmm} / 2.2 \\
\mathrm{krm} / 2.2\end{array}$ & 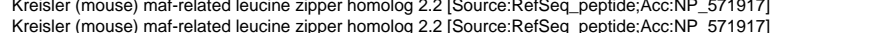 & $\begin{array}{l}-76 \\
-43\end{array}$ & $\begin{array}{l}-71 \\
-38\end{array}$ & 1 & $\begin{array}{l}\text { ggcgtg } \\
\text { acgat }\end{array}$ \\
\hline ENSDARG00000044176 & ENSDART00000064858 & $\begin{array}{l}\text { Dr.23470.1.S1 s at,Dr.8198.1.A1_at } \\
\text { Dre at }\end{array}$ & krml2.2. & Kreisler (mouse) mat-related leucine zipper homolog 2.2 [Source:RefSeq peptide;Acc:NP 571917 ] & -3256 & -3251 & $\begin{array}{c}1 \\
-1\end{array}$ & $\begin{array}{l}\text { aggegi } \\
\text { agcatg }\end{array}$ \\
\hline ENSDARG00000044176 & ENSDART00000064858 & Dr.23470.1.S1 s at,Dr.8198.1.A1 at & $\mathrm{krm} / 2.2 \mathrm{r}$ & Kreisler (mouse) maf-related leucine zipper homolog 2.2 [Source:RefSeq peptide:Acc:NP 571917 ] & -1753 & -1748 & -1 & 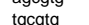 \\
\hline ENSDARG00000044176 & ENSDART00000064858 & Dr.23470.1.S1_s at,Dr.8198.1.A1_at & $\mathrm{krm} / 2.2$ & Kreisler (mouse) mat-related leucine zipper homolog 2.2 [Source:RefSeq_peptide & -76 & & 1 & ggcgtg \\
\hline ENSDARG00000044176 & ENSDART00000064858 & Dr.23470.1.S1 sat,Dr.8198.1.A1 at & $\mathrm{k} \mathrm{km} / 2.2$ & Kreisler (mouse) maf-related leucine zipper homolog 2.2 [Source:RefSeq peptide:Acc:NP 571917 ] & -43 - & -38 & 1 & $\begin{array}{l}\text { agctg } \\
\text { acgtg }\end{array}$ \\
\hline ENSDARG00000044613 & ENSDART00000014927 & Dr.14041.1.S1-at & NP_001005976.1 & hypothetical protein LOC449803 [Source:RefSeq_peptide;ACC:NP_001005976] & -3600 & -3595 & 1 & ggcgtg \\
\hline ENSDARG00000044613 & ENSDART00000014927 & Dr.14041.1.S1_at & NP_-001005976.1 & hypothetical protein LOC449803 [Source:RefSeq_peptide;ACc:NP_001005976] & -3377 & -3372 & 1 & cgcgtg \\
\hline ENSDARG00000044613 & ENSDART00000014927 & Dr.14041.1.S1_at & NP_-001005976.1 & hypothetical protein LOC449803 [Source:RefSeq_peptide;Acc:NP_001005976] & -58 & -53 & -1 & tgcgtg \\
\hline ENSDARG000000044613 & ENSDART00000014927 & Dr.14041.1.S1_at & NP_001005976.1 & hypothetical protein LOC449803 [Source:RefSeq_peptide;Acc:NP_001005976] & -39 & -34 & 1 & agcgtg \\
\hline ENSDARG000000044625 & ENSDART00000065562 & Dr.14268.1.A1_at,Dr.18893.1.S1_at & zgc:92226 & hypothetical protein LOC492341 [Source:RefSeq_peptide;Acc:NP_001007308] & -4982 & -4977 & 1 & tgcgtg \\
\hline $\begin{array}{l}\text { ENSDARG0000000046625 } \\
\text { ENSDARG00000044936 }\end{array}$ & $\begin{array}{l}\text { ENSDARTO00000006562 } \\
\text { ENSDATT00000066051 }\end{array}$ & $\begin{array}{l}\text { Dr.14268.1.A1_at,Dr.18893.1.S1_at } \\
\text { Dr 71101S1_at }\end{array}$ & $\begin{array}{l}\text { zgc::92226 } \\
\text { zac } 77614\end{array}$ & 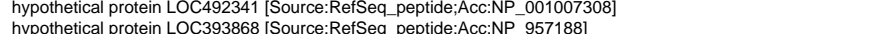 & $\begin{array}{r}-251 \\
-3792\end{array}$ & $\begin{array}{l}-246 \\
-3787\end{array}$ & 1 & agcgtg \\
\hline ENSDARG000000044936 & $\begin{array}{l}\text { ENSDARTO00000066051 } \\
\text { ENSDART0000066051 }\end{array}$ & $\begin{array}{l}\text { Di.r.110.1.S1_at } \\
\text { Dr.7110.1.S1 at }\end{array}$ & $\begin{array}{l}\text { zact:7/1014 } \\
\text { zgc:77614 }\end{array}$ & 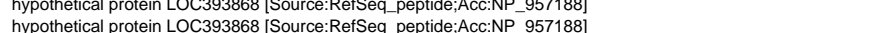 & 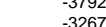 & -3262 & & tgctg \\
\hline ENSDARG00000044936 & ENSDART00000066051 & $\begin{array}{l}\text { Dr.7110.1.S1_at at } \\
\text { Dr.s. }\end{array}$ & zgc: 77614 & hypothetical protein LOC393868 [Source:RefSeq_peptide;AcC:NP_957188] & -4066 & -4061 & 1 & 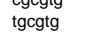 \\
\hline ENSDARG00000044975 & ENSDART00000030103 & Dr.1361.1.S1_at & & & -3650 & -3645 & 1 & cgcgtg \\
\hline ENSDARG00000044975 & ENSDART00000030103 & Dr.1361.1.S1_at & & & -2276 & -2271 & 1 & agcgtg \\
\hline ENSDARG00000044975 & ENSDART00000030103 & Dr.1361.1.S1_at & & & -1621 & -1616 & -1 & \\
\hline ENSDARG00000044975 & ENSDART00000030103 & Dr.1361.1.S1_at & & & -4656 & -4651 & -1 & tgcgtg \\
\hline ENSDARG00000044975 & ENSDART00000030103 & Dr.1361.1.S1_at & & & -50 & -45 & 1 & agcgtg \\
\hline ENSDARG00000044983 & ENSDART00000066126 & Dr.7110.1.S1_at & & & -3372 & -3367 & 1 & agcgtg \\
\hline ENSDARG000000045180 & ENSDART00000066430 & Dr.20277.1.A1_at & NP_997785.1 & actin, alpha 2, smooth muscle, aorta [Source:RefSeq_peptide;Acc:NP_997785] & -2999 & -2994 & 1 & agcgtg \\
\hline ENSDARG000000045230 & ENSDART00000066506 & Dr.956.1.S1_at & NP_001002695.1 & zgc:92631 [Source:RefSeq_peptide;ACc:NP__001002695] & -3672 & -3667 & 1 & tgcgtg \\
\hline ENSDARG000000045230 & 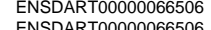 & Dr.956.1.S1_at & NP_001002695.1 & zgc:92631 [Source:RefSeq_peptide;Acc:NP_001002695] & -3639 & -3634 & $-{ }^{-1}$ & cgcgtg \\
\hline $\begin{array}{l}\text { ENSDARG0000000052330 } \\
\text { ENSDARG0000004530 }\end{array}$ & $\begin{array}{l}\text { ENSDARTT00000066506 } \\
\text { ENSDAT0000006506 }\end{array}$ & $\begin{array}{l}\text { Dr.955.1.S1_at } \\
\text { Dr.956.1S1 at }\end{array}$ & NP_001002695.1 & zgc:92631 [Source:Reefeq_pepptide;Acc:NP_001002695] & $\begin{array}{r}-3033 \\
-1880\end{array}$ & $\begin{array}{r}-3028 \\
-1875\end{array}$ & -1 & $\begin{array}{l}\text { agcgtg } \\
\text { gacotga }\end{array}$ \\
\hline ENSDARG00000045230 & ENSDART00000066506 & $\begin{array}{l}\text { Dr.956.1.S1_at } \\
\text { Dr.st }\end{array}$ & NP_001002695.1 & 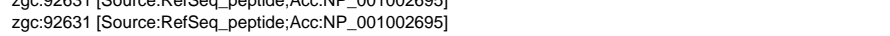 & -294 & $\begin{array}{l}-1889 \\
-289\end{array}$ & -1 & $\begin{array}{l}\text { geglig } \\
\text { tgctg }\end{array}$ \\
\hline 0000045230 & ENSDART00000066506 & Dr.956.1.S1-at at & $N P=001002695.1$ & zac:92631 [Source:RefSeg peptide:Acc:NP 001002695 ] & $-22>>-20$ & -17 & -1 & gacota \\
\hline ENSDARGC & ENSDA & Dr.956 & $N P_{0010026951}$ & Acc:NP- 001002695 & -4498 > & -4493 & -1 & tacatg \\
\hline ENSDARG00000045230 & 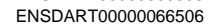 & Dr.956.1.151 at & NP 001002695.1 & 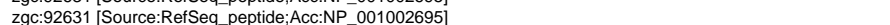 & -4452 & -4447 & -1 & $\begin{array}{l}\text { tgcctg } \\
\text { tgctg }\end{array}$ \\
\hline ENSDARG00000045407 & ENSDART00000066773 & Dr.10879.1.Ā1_at & $\mathrm{NP}^{-0} 001018414.1$ & hypothetical protein LOC553602 [Source:RêfSeq_peptide:Acc:NP_001018414] & -3212 & -3207 & -1 & tgcgtg \\
\hline ENSDARG00000045407 & ENSDART00000066773 & Dr.10879.1.A1_at & NP_-001018414.1 & hypothetical protein LOC553602 [Source:RefSeq_peptide;ACc:NP_-001018414] & -877 & -872 & 1 & agcgtg \\
\hline ENSDARG00000045407 & ENSDART00000066773 & Dr.10879.1.A1_at & NP_-001018414.1 & hypothetical protein LOC553602 [Source:RefSeq_peptide;ACc:NP_001018414] & -100 & -95 & -1 & agcgtg \\
\hline ENSDARG00000045567 & ENSDART00000067004 & $\begin{array}{l}\text { Dr.13994.1.S1_X_at,Dr.13994.2.A1_a_at,Dr.13994.1.S1_at,Dr.13994.2.A1_x_- } \\
\text { at,Dr.2819.1.A1_at }\end{array}$ & zgc:101086 & hypothetical protein LOC445172 [Source:RefSeq_peptide;Acc:NP_001003566] & -2856 & -2851 & -1 & $\operatorname{tgcgtg}$ \\
\hline ENSDARG00000045567 & ENSDART00000067004 & 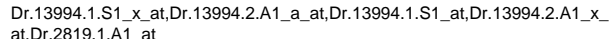 & $z a c \cdot 101086$ & hypothetical protein LOC445172 [Source:RefSeq_peptide;ACc:NP_001003566] & & & 1 & anc \\
\hline ENSDARG00000045567 & ENSDART00000067004 & at,Dr.2819.1.A1_at & zgc:101086 & & -2844 & -2839 & -1 & agcgtg \\
\hline ENSDARG00000045567 & ENSDART00000067004 & 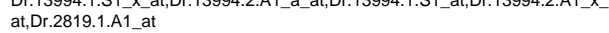 & zgc:101086 & hypothetical protein LOC445172 [Source:RefSeq_peptide;Acc:NP_001003566] & & -2 - & -1 & \\
\hline ENSDARG00000045585 & ENSDART00000067027 & Dr.13689.1.S1_at & zgc:66449 & Unkn & & & & \\
\hline ENSDARG00000045585 & ENSDART00000067027 & Dr.13689.1.S1_at & zgc:66449 & Unkr & -4153 & & -1 & \\
\hline 45678 & ENSDAR & Dr.15819.1.A1 ${ }^{-a t . D r}$ & & tryptophan hydroxylase 1 , like [Source:RefSeq peptide; Acc:NP & -3633 & -3628 & 1 & $\begin{array}{l}\text { tgcatg } \\
\text { tgcta }\end{array}$ \\
\hline ENSD & ENSDART00000067159 & Dr.15819.1.A1 at,Dr.17776.1.A1 at & tph11 & tryptophan hydroxylase 1, like [Source:RefSeq peptide;Acc:NP 001001843 ] & -1855 & -1850 & 1 & agcgtg \\
\hline ENSDARG00000045678 & ENSDART00000067159 & Dr.15819.1.A1_at,Dr.17776.1.A1_at & tph11 & tryptophan hydroxylase 1 , like [Source:RefSeq_peptide;Acc:NP_001001843] & -1704 & -1699 & -1 & agcgtg \\
\hline ENSDARG00000045678 & ENSDART00000067159 & Dr.15819.1.A1_at,Dr.17776.1.A1_at & tph11 & tryptophan hydroxylase 1, like [Source:RefSeq_peptide;Acc:NP_001001843] & -240 & -235 & -1 & agcgtg \\
\hline ENSDARG00000045678 & ENSDART00000067159 & Dr.15819.1.A1_at,Dr.17776.1.A1_at & tph11 & tryptophan hydroxylase 1, like [Source:RefSeq_peptide;Acc:NP_001001843] & -56 & -51 & 1 & cgcgtg \\
\hline
\end{tabular}




Transcript ID

$\begin{array}{ll}\text { ENSDARG000000001953 } & \text { ENSDART00000001053 } \\ \text { ENSDARTO000000324 }\end{array}$

ENSDARG00000002667 ENSDART00000003004

ENSDARG00000002667 ENSDART00000003004 $\begin{array}{ll}\text { ENSDARG000000003323 } & \text { ENSDARTO0000001756 } \\ \text { ENSDARG00000003323 } & \text { ENSDART000000175 }\end{array}$

ENSDARG00000004296 ENSDART0000002702 ENSDARTT00000017312 ENSDARG00000008835 ENSDART0000002128 ENSDARG00000008835 ENSDART0000002128 ENSDART0000004472

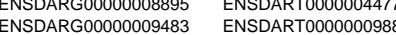
ENSDARG00000009483 ENSDARTO0000000988 ENSDARG000000013613 $\quad$ ENSDARTT00000001840

$\begin{array}{ll}\text { ENSDARG00000013646 } & \text { ENSDART0000001966 } \\ \text { ENSDARG00000014209 } & \text { ENSDART0000002320 }\end{array}$ $\begin{array}{ll}\text { ENSDARG00000014209 } & \text { ENSDART00000023208 } \\ \text { ENSDARG00000015866 } & \text { ENSDART00000019110 }\end{array}$ ENSDARG00000015866 ENSDART00000058963 ENSDARG000000015866 $\quad$ ENSDARTO0000005896 $\begin{array}{ll}\text { ENSDARG00000016448 } & \text { ENSDART00000014979 } \\ \text { ENSDARG00000016448 } & \text { ENSDART00000014979 }\end{array}$ ENSDARG00000016448 ENSDART00000014979 ENSDARG00000016825 ENSDART0000005023 ENSDARG00000016825 ENSDART00000050238 ENSDARG00000016825 ENSDART0000005023 ENSDART0000005023 ENSDARO00000016825 ENSDART0000005023 ENSDARTO000005023

ENSDARG00000017289 ENSDART00000047865 ENSDARG00000017289 ENSDART00000050670 ENSDARG00000017289 ENSDART00000047865 ENSDARG00000017289 ENSDART00000050670 ENSDARG00000017619 ENSDARG00000017624 ENSDART00000012644 ENSDARG000000018529 ENSDART0000002054 ENSDARG00000020485 ENSDARTO0000011573 ENSDARG00000021443 ENSDART0000001415 ENSDARG000000023963 ENSDART00000036075 ENSDARG00000024892 ENSDART00000033228 ENSDARG00000024892 ENSDART00000033228 ENSDARG00000024892 ENSDART0000003322 ENSDARG00000024892 ENSDART00000033228 ENSDARG00000025400 ENSDART00000044869 ENSDARG00000025400 ENSDART00000046418 ENSDARG00000025400 ENSDART00000061150 ENSDARG00000025400 ENSDART00000061161 ENSDARG000000025400 $\quad$ ENSDARTO00000061165 $\begin{array}{ll} & \text { ENSDARTO000003820 } \\ \text { ENSDARG000000026090 } & \text { ENSDARTO000003530 }\end{array}$ ENSDARG00000026680 ENSDART00000036600 ENSDARG00000026680 ENSDART0000003660 ENSDARG00000027088 ENSDART00000039267

ENSDARG00000027924 ENSDART00000051147 ENSDARG00000027924 ENSDART00000051147 ENSDARG00000027924 ENSDART00000051147
Affy IDs

Dr.7626.1.A1_at,Dr.7626.2.S1_at
Dr.23470.1.S1_s_at,Dr.8198.1.A1_at

Dr.23470.1.S1_s_at,Dr.8198.1.A1_at

Dr.12575.1.S1_at

Dr.12575.1.S1_at
Dr.2426.1.S1_at

Dr.26469.1.S1_at
D.24233.S1_at
D.24.1. at

Dr.4412.13.A1_x_at

Dr.4412.13.A1_- $\mathrm{x}$-at

Dr.1619.1.A1 at,Dr.1619.1.A1 at,Dr.1619.1.A1_at

Dr.25378.1.A1_at,Dr.24858.1.S1_at

Dr.16554.1.S1 at

Dr.16504.1.A1_at

Dr.15930.1.A1_at

Dr.728.4.S1_at,Dr.728.4.S1_at,Dr.728.4.S1_at

Dr.728.4.S1_at,Dr.728.4.S1_at,Dr.728.4.S1_at

Dr.2978.1.S1_at
Dr.2978.1.S1_at

Dr.25009.6.A1_a_at,Dr.25009.1.S1_a_at

Dr.25009.6.A1_a_at,Dr.25009.1.S1_a_a

Dr.25009.6.A1 a atD. Dr.25009.1.S1 a-

Dr.25009.6.A1_a_at,Dr.25009.1.S1_a_a

Dr.25009.6.A1_a_aa,Dr.25009.1.S1_a_ad D.,Dr.3025.3.31_at 2531.1. 1__t,Dr.25379.1.S1_at,Dr.3025.3.S1_at,Dr.25331.1.S1_at,Dr.25379.1.S1_a t,Dr.3025.3.s. at at

_25379.1S1_at.Dr.3025.3.S1_at,Dr.25331.1.S1_at,Dr.25379.1.S1

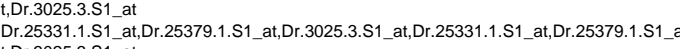
t.,Dr.3025.3.S1 ${ }_{1}$ at

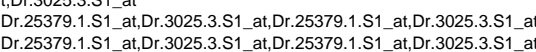

Dr.4387.1.S1_at
Dr.4249.1.S1_at

Dr.25285.1.11_at,Dr.11211.1.S1_at

Dr.10314.1.S1_a_at

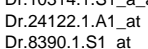

Dr.8390.1.S1_at
Dr.913.1.S1_at

Dr.3025.2.S1_at,Dr.3025.3.S1_at,Dr.25331.1.S1_at,Dr.25379.1.S1_at,Dr.3025.2.S1_at,D r.3025.3.S1_at,Dr.25379.1.S1_at,Dr.3025.1.A1_at,Dr.3025.3.S1_at at
Dr.3025.2.S1_at,Dr.3025.3.S1_at,Dr.25331.1.S1_at.Dr.25379.1.S1_at,Dr.3025.2.S1_at,D

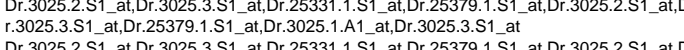
Dr.3025.2.S1_at,Dr.3025.3.S1_at,Dr.25331.1.S1_at,Dr.25379.1.S1_at,Dr.3025.2.S1_at,D r.3025.3.S1_at,Dr.25379.1.S1_at,Dr.3025.1.A1_at,Dr.3025.3.S1_at
Dr.3025.2.S1_at,Dr.3025.3.S1_at,Dr.25331.1.S1__t,Dr.25379.1.S1_at,Dr.3025.2.S1_at,D

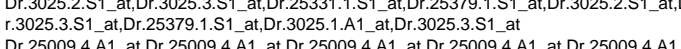
Dr.25009.4.A1__at,Dr.25009.4.A1_at,Dr.25009.4.A1_at,Dr.25009.4.A1_at,Dr.25009.4.A1
at Dr.25009.4.A1_at,Dr.25009.4.A1_at,Dr.25009.4.A1_at,Dr.25009.4.A1_at,Dr.25009.4.A1_ Dr.25009.4.A1_at,Dr.25009.4.A1_at,Dr.25009.4.A1_at,Dr.25009.4.A1_at_Dr.25009.4.A1
at Dr.25009.4.A1_at,Dr.25009.4.A1_at,Dr.25009.4.A1_at,Dr.25009.4.A1_at,Dr.25009.4.A1 Dr.25009.4.A1_at,Dr.25009.4.A1_at,Dr.25009.4.A1_at,Dr.25009.4.A1_at,Dr.25009.4.A1 Dr.9478.1.S1_at

Dr.26139.1.A1_at,Dr.26139.1.A1 x a

Dr.26139.1.A1_at,Dr.26139.1.A1_x_at

Dr.1192.1.S1_at,Dr.1192.1.S1_a_at

Dr.1192.1.S1_at,Dr.1192.1.S1_a_at
Dr.1192.1.S1_at,Dr.1192.1.S1_a_at

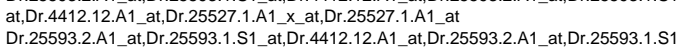
t,Dr.4412.12.A1__at,Dr.25527.1.A1____at,Dr.25527.1.A1_at

D.25593.2.A1_at,Dr.25593.1.S1_at,Dr.4412.12.A1_at,Dr.25593.2.A1_at,Dr.25593.1.S1

vg

lipt

zgc:73223
Description

Znc finger CCCH type domain containing 5 [Source:RetSeq_peptide;Acc:NP_956530]

2gc: 73257 [Source:RefSeq__eptide;ACc:NP_998562]
Kreisler (mouse) mat-related leucine zipper homolog

Kreisler (mouse) maf-related leucine zipper homolog 2.2
[Source:RefSeq_peptide;Acc:NP_571917]

$\begin{array}{ll}\text { dcp1a } & \text { decapping enzyme [Source:RefSeq_peptide:Acc:NP_878313] } \\ \text { dcp1a } & \text { decapping enzyme [Source:RefSea_peptide:Acc:NP 878313] }\end{array}$

similar to alpha-1-microglobulin/bikunin precursor [Source-RefSea_peptide:Acc:NP _957412]

2gc:66321 5 sp4 transcription factor [Source:RefSeq_peptide;Acc:NP_956418]

$\begin{array}{ll}\text { zgc:110540 } & \text { deoxycytidine kinase [Source:RefSeq_peptide:Acc:NP_001014374] } \\ \text { zgc:110540 } & \text { deoxycytidine kinase [Source:RetSeq_peptide:Acc:NP_001014374] }\end{array}$

wu.tc12604 protein-glutamine gamma-glutamyltransferase [Source:RefSeq_peptide;ACc:NP_99782

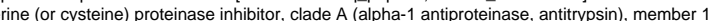

$\begin{array}{ll}\text { serpina1 } & \text { [Source:RefSeq_peptide;Acc:NP_001013277] } \\ \text { NP_001017836.1 hypothetical protein LOC550534 [Source:RefSeq_peptide;AAcc:NP_001017836] }\end{array}$

NP_739573.1

NP_-739573.

$\mathrm{NP}_{-739573.1}$

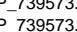

NP_-955943.1

Vitellogenin 3 (Fragment). [Source:Uniprot/SPTREMBL;Acc:Q9DFT9]

Vitellogenin 3 (Fragment). [Source:Uniprot/SPTREMBL;ACc:Q9DFT9]
Vitellogenin 3 (Fragment). [Source:Uniprot/SPTREMBL:ACC:QDDFT9]

vitellogenin 1 [Source:RefSeq_peptide;Acc:NP_739573]
vitellogenin 1 [Source:RefSeq_peptide;Acc:NP_739573]

vitellogenin 1 [Source:RefSeq_peptide; Acc:NP- 739573

vitellogenin 1 [Source:RefSeq peptide;Acc:NP 739573

tellogenin 1 [Source:RefSeq_peptide;Acc:NP_739573

vitellogenin 1 (Source:RerSeq_peptide;Acc:NP_-739573

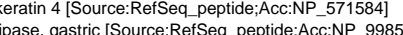

hypothetical protein MGC73223 [Source:RefSeq_peptide;ACc:NP 957126

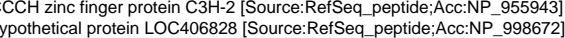

cytochrome P450, family 1, subfamily A [Source:RefSeq_peptide:Acc:NP_57195

solute carrier family 25 (mitochondrial carrier, brain), member 14

Source:RefSeq_peptide;Acc:NP_956458]

solute carrier family 25 (mitochondrial car
[Source:RefSeq_peptide;Acc:NP__556458]

prostaglandin D2 synthase [Source:RefSeq_peptide;ACc:NP 9998799$]$

prostaglandin D2 synthase [Source:RefSeq_peptide;AAcc:NP_998799]

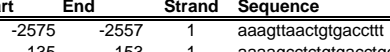

aaagttactgtgaccttr
aaaagactctgtgaaccto

$\begin{array}{lllll}-1337 & -1319 & -1 & \text { tccagcaactgtgaccaac }\end{array}$

$\begin{array}{llll}-1337 & -1319 & -1 & \text { tccagcaactgtgaccaac } \\ -1949 & -1931 & 1 & -1\end{array}$

$\begin{array}{llll}-1949 & -1931 & 1 & \text { tcaaggacccatgaccc9 } \\ -381 & -363 & 1 & \text { gtgagtaaacatgactga }\end{array}$

$\begin{array}{llll}-4061 & -4043 & -1 & \text { atagtgtaatatgacccta } \\ -1496 & -1478 & 1 & \text { togatcactgtgaccos }\end{array}$

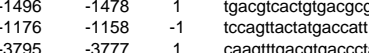

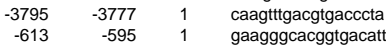

$\begin{array}{llll}-3866 & -3848 & 1 & \text { caacatcactctgaccaga } \\ -3872 & -3854 & 1 & \text { caacatcactctgaccaga } \\ -2635 & -2617 & 1 & \text { tcagttcattrgatctag }\end{array}$

tcagttcatttgatctag

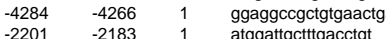

$\begin{array}{llll}-267 & -249 & -1 & \text { ttatgggacttgaccttt }\end{array}$

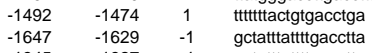

$\begin{array}{llll}-1945 & -1927 & -1 & \text { gctatttatttgacctta } \\ -1647 & -1629 & -1 & \text { gctatttattlgacctta }\end{array}$

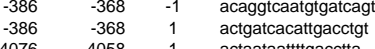

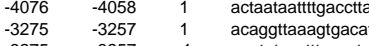

$-1057$ 
ENSDARG00000027924 ENSDART00000051147 ENSDARG00000027924 ENSDART00000051147 ENSDARG00000027924 ENSDART00000051147 $\begin{array}{ll}\text { ENSDARG00000029086 } & \text { ENSDART0000004192 } \\ \text { ENSDARG00000030236 } & \text { ENSDART00000011928 }\end{array}$ $\begin{array}{ll}\text { ENSDARG00000030236 } & \text { ENSDART00000011928 } \\ \text { ENSDARG00000030662 } & \text { ENSDART00000048550 }\end{array}$ ENSDARG00000031664 ENSDART0000004933 $\begin{array}{ll}\text { ENSDARG00000033735 } & \text { ENSDART00000049874 } \\ \text { ENSDARG00000033735 } & \text { ENSDART0000004987 }\end{array}$ ENSDARTO00000049874 ENSDARG00000033735 ENSDARG00000033735 ENSDART000000508 ENSDARTO000000119 $\begin{array}{ll}\text { ENSDARG000000035743 } & \text { ENSDARTO0000005185 } \\ \text { ENSDARG00000036481 } & \text { ENSDART0000005300 }\end{array}$

ENSDARG00000036517 ENSDART00000053070 ENSDARG00000036519 ENSDARG00000036527 ENSDARG00000036531 ENDARG00003551

ENSDARG00000036776 ENSDARG0000003677 ENSDARG0000003689 ENSDARG000000368 ENSDARG0000003972 ENSDARG0000003927 ENSDARG00000040251 ENSDARG0000004025 ENSDARG00000040295

ENSDARG00000040050 ENSDART00000044076 ENSDARG00000041639 ENSDART00000044076 ENSDARG00000041639 ENSDART00000044076 ENSDARG00000041639 ENSDART00000044076 ENSDARG00000041653 ENSDARTO00000061043 ENSDARG00000041774 ENSDART00000061223 ENSDARG000000042035 ENSDART00000061601 ENSDART00000061618 ENSDARG00000042391 ENSDART0000006215 ENSDARG00000043618 ENSDART0000006404

ENSDARG00000043670 ENSDART00000024462 $\begin{array}{ll}\text { ENSDARG000000043960 } & \text { ENSDARTO00000064542 } \\ \text { ENSDARG00000043990 } & \text { ENSDARTO0000064579 }\end{array}$ $\begin{array}{ll}\text { ENSDARG00000043990 } & \text { ENSDARTT000000645 } \\ \text { ENSDARG00000044002 } & \text { ENSDARTO00000645 }\end{array}$ $\begin{array}{ll}\text { ENSDARG00000044002 } & \text { ENSDART00000064591 } \\ \text { ENSDARG00000044125 } & \text { ENSDART0000006478 }\end{array}$ ENSDARG00000044176 ENSDART00000064858 ENSDARG00000044176 ENSDART00000064858 ENSDARG00000044613 ENSDART00000014927 $\begin{array}{ll}\text { ENSDARG00000044936 } & \text { ENSDART0000006605 } \\ \text { ENSDARG00000044936 } & \text { ENSDART0000006605 }\end{array}$ ENSDARG00000045230 ENSDART00000066506
at,Dr.4412.12.A1_at,Dr.25527.1.A1_x at,Dr.25527.1.A1_at

Dr.25593.2.A1_at,Dr.25593.1.S1_at,Dr.4412.12.A1_at,Dr.25593.2.A1_at,Dr.25593.1.S1 at,Dr.4412.12.A1_at,Dr.25527.1.A1_X_at,Dr.25527.1.A1_at at 0 Dr.25593.2.A1_at,Dr.25593.1.S1 at,DPr.4412.12.A1 at,Dr.25593.2.A1_at,Dr.25593.1.S1

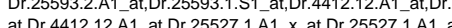

Dr.1282.1.S1_at
Dr.1368.7.S1_at Dr.1368.7.S1_at Dr.2973.1.A1_at,Dr.2973.1.A1_at
Dr.2973.1.A1_at,Dr.2973..A1 a $\begin{array}{ll} & \\ & \end{array}$ Dr.2973.1.A1_at,DV.2973.1.A1_a Dr.2973.1.A1_at,Dr.2973.1.A1_at Dr.25009.6.A1_a_a_a
Dr.13775.1.S1_at Dr.13775.1.S1_at
Dr.14748.1.S1_at Dr.6550.1.A1_at
Dr.1411.1.S1_at

Dr.1411.1.S1_at Dr.25155.1.S1_s_a Dr.1411.1.S1_a
Dr.8723.1.S1_a Dr.8723.1.S1
Dr.1411.1.S1 Dr.1411.1.S1_at

Dr.16380.1.A1_at

Sox21a SRY-box 21 [Source:RefSeq_peptide:Acc:NP _571361]

Q4Z8P3_BRARE Neutrophil cytosolic factor 1. [Source:Uniprot/SPTREMBL:Acc: Q4Z8P

Q4Z8P3BRARE Neutrophil cytosolic factor 1. SSource:UniprotsPTREMBL;Acc:Q4Z8P

(1.

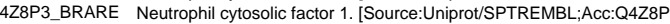

Unknown (protein for MGC:63985) [Source:RefSeq peptide-Acc:NP 956252]

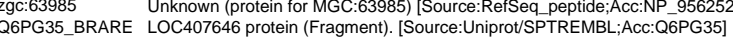

si:xx-by187g17 1 Novel protein similar to zebratish hemoglobin alpha-adult 1 (Hbaa).

hbaa1 Hemoglobin alpha chain (Alpha globin aa1). [Source:Uniprot/SWISSPROT;Acc:Q90487]

hbaa1 Hemoglobin alpha chain (Alpha globin aa1). [Source:Uniprot/SWISSPROT;ACc:Q90487]

Hemoglobin beta-1 chain (BetaA1 globin). [Source:Uniprot/SWISSPROT;AAc:QQ99]
si:xx-by187g17.1 hemoglobin alpha adult-1 (hbaa), mRNA [Source:RefSeq_dna;ACc:NM__31257]

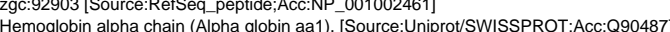

ba2 Hemoglobin beta-1 chain (BetaA1 globin). [Source.Uniprots

NP_001004540.1 aldehyde dehydrogenase 8 family, member A1 [Source:RefSeq_peptide;Acc:NP_001004540

Dr.16206.1.A1_at,Dr.16206.1.A1_s_at

Dr.16206.1.A1_at,Dr.16206.1.A1_s_a
Dr.16206.1.A1_at,Dr.16206.1.A1_s_at
Dr.16206.1.A1_at,D.16206.1.A1s sa

Dr.16206.1.A1_at,Dr.16206.1.A1_s_a

Dr.24341.1.S1_at Dr.3581.1.S1_at,Dr.3581.1.S1_a_at

Dr.4797.1.S1_at

Dr.4048.1.S1_at

Dr.4048.1.S1_at

Dr.4048.1.S1_at

Dr.1246.1.S1_at

Dr.20533.1.A1_at
Dr.4212.1.S1_at

NP_010045401 aldehyde dehydrogenase 8 family, member A1 [Source:RefSeq_peptide;Acc:NP_001004540]

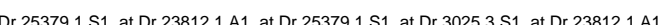

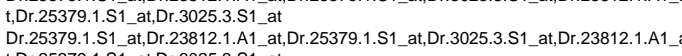
t,Dr.25379.1.S1_at,Dr.3025.3.S1_at

D. 25r.25379.1 s1_at,Dr 3025.1.A1_at,Dr.25379.1.S1_at,Dr.3025.3.S1_at,Dr.23812.1.A1_a Dr.25379.1.S1_at,Dr.23812.1.1.A1__at,Dr.25379.1.S1_at,Dr.3025.3.S1_at,Dr.23812.1.A1_a (D.25379.1.S1_at,Dr.3025.3.S1_ea

25331.1.S1_at,Dr.25379.1.S1_at,Dr.25331.1.S1_at,Dr.25379.1.S1_at

Dr.25009.6.A1_a_a
D.r.7111.1.1_Lat

Dr.7110.1.S1_at
Dr.7110.1.S1 at

Dr.14748.1.S1_at

Dr.7236.1.A1_at,Dr.7236.1.A1_at

Dr.7236.1.A1_at,Dr.7236.1.A1_at

Dr.6147.1.A1_at

Dr.7806.1.A1_at

Dr.11609.1.S1_at,Dr.11609.1.S1_at

Dr.23470.1.S1_s_at,Dr.8198.1.A1_at

Dr.23470.1.S1_S_at,Dr.8198.1.A1_at

Dr.14041.1.S1_at
Dr.7110.1.S1_at

Dr.7110.1.S1_at zgc:776

zgc:77614

作

(sine (or cysteine) proteinase inhibitor clade A (alpha-1 antiproteinase, antitrypsin), member 1

NM_001013259.1_ (serpina1), mRNA [Source:RefSeq dna:Acc:NM _00101325

hypothetical protein LOC406587 [Source:RefSeq_peptide;Acc:NP_999913]

NP_001002187.1 hypothetical protein LOCC431734 [Source:RefSeq_peptide;ACC:NP_001002187]

$\begin{array}{ll}\text { 2gc.92903 } & \text { Kreister (mouse) maf-related leucine zipper homolog } 2.2 \\ \text { krml2.2 } & \text { [Source:RefSeq_peptide;Acc:NP_571917] }\end{array}$

$\begin{array}{ll}\text { Kreisler (mouse) maf-related leucine zipper } \\ \text { krml2.2 } & \text { [Source:RefSeq_peptide;Acc:NP_571917] }\end{array}$

NP_001005976.1 hypothetical protein LOC449803 [Source:RefSeq_peptide;ACc:NP_001005976]

zgc: 777614 hypothetical protein LOC393868 [Source:RefSeq_peptide;ACC:NP_-057188

$\begin{array}{lll}\text { zgc: } 77614 & \text { hypothetical protein LoC } 393868 \text { [Source:RefSeq_peptid } \\ \text { NP _001002695.1 } & \text { zgc:92631 [Source:RefSeq_peptide:ACc:NP_001002695] }\end{array}$ hypothetical protein LOC393868 [Source:RefSeq_peptide:Acc:NP_9957188
zgc:63985 (zgc:63985), mRNA [Source:RefSeq_dna:Acc:NM_199958]

\begin{tabular}{|c|c|c|c|}
\hline \multirow{2}{*}{$\begin{array}{r}-456 \\
-224\end{array}$} & 4543 & -1 & caagtttgacgtgacccta \\
\hline & -2229 & -1 & taaaatcggtttgaccttt \\
\hline-4561 & -4543 & -1 & caagtttgacgtgacccta \\
\hline $\begin{array}{r}-218 \\
-3947 y-2\end{array}$ & -200 & -1 & gtaattcaatatgaccaga \\
\hline $\begin{array}{l}-3947 \\
-8600\end{array}$ & $\begin{array}{l}-3929 \\
-1842\end{array}$ & $\begin{array}{l}1 \\
1\end{array}$ & gccattcacactgacctga \\
\hline-4888 & $\begin{array}{l}-1842 \\
-4870\end{array}$ & $\begin{array}{l}1 \\
1\end{array}$ & $\begin{array}{l}\text { gcacgtgcctgtgacctgg } \\
\text {.tagaagcacttaacctc }\end{array}$ \\
\hline-3790 & -3772 & -1 & aaagcaaactgtgacctag \\
\hline-2927 & -2909 & -1 & tgaggctatagtgacccag \\
\hline-1290 & -1272 & 1 & tgatgctaatgtgacctta \\
\hline $\begin{array}{l}-3845 \\
-2982\end{array}$ & $\begin{array}{l}-3827 \\
-2964\end{array}$ & -1 & aaagcaaactgtgacctag \\
\hline $\begin{array}{l}-2982 \\
-1345\end{array}$ & $\begin{array}{l}-2964 \\
-1327\end{array}$ & $\begin{array}{c}-1 \\
1\end{array}$ & $\begin{array}{l}\text { tgaggctatagtgacccag } \\
\text { tgatctcaatatgacctta }\end{array}$ \\
\hline-179 & -161 & 1 & $\begin{array}{l}\text { tgatgctaaatgigactrat } \\
\text { gcaggccaggttaacctaa }\end{array}$ \\
\hline-2271 & -2253 & -1 & ttggtgaactttgacctgc \\
\hline $\begin{array}{l}-4293 \\
-806\end{array}$ & $\begin{array}{r}-4275 \\
-78\end{array}$ & -1 & acagtgagctctgaccttg \\
\hline & & & aaatggaaaattgaccttc \\
\hline-1664 & -1646 & 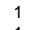 & tccgcgcggtgtgaccttc \\
\hline $\begin{array}{l}-3550 \\
-1642\end{array}$ & $\begin{array}{l}-3232 \\
-1624 \\
\end{array}$ & 1 & acaattcactttgacttta \\
\hline & & & tccgcgcggtgtgaccttc \\
\hline & - -4649 & -1 & tccgcgcggtgtgaccttc \\
\hline & $\begin{array}{l}-1666 \\
-255\end{array}$ & 1 & tccgcgcggtgtgaccttc \\
\hline 3243 & -3225 & 1 & $\begin{array}{l}\text { Iccagagcicatclgactrgc } \\
\text { acaattcacttgacttra }\end{array}$ \\
\hline-1636 & -1618 & 1 & $\begin{array}{l}\text { acdatcadcigacida } \\
\text { tccgcgcggtgtgaccttc }\end{array}$ \\
\hline-4257 & -4239 & -1 & tccgcgcggtgtgaccttc \\
\hline-2837 & -2819 & 1 & acaaacttctttgactgt \\
\hline & -531 & -1 & ataagctacaatgacctaa \\
\hline $\begin{array}{l}-3911 \\
-1045\end{array}$ & -3893 & -1 & taaagttatattgacccca \\
\hline $\begin{array}{l}-3911 \\
-3911\end{array}$ & $\begin{array}{l}-1027 \\
-3893\end{array}$ & $\begin{array}{l}-1 \\
-1\end{array}$ & acagaaagttgtgaccaaa \\
\hline & -1027 & -1 & $\begin{array}{l}\text { taaagagttatattgacccca } \\
\text { acagaaastrtagaccaaa }\end{array}$ \\
\hline-369 & -3673 & -1 & $\begin{array}{l}\text { acagaaaagttgtgaccaaa } \\
\text { ataagtaatcctgacccgt }\end{array}$ \\
\hline 4485 & -4467 & -1 & gcgaataattttgacctta \\
\hline-3957 & -3939 & -1 & tttgttaactttgacctgc \\
\hline$-90,-5$ & -890 & -1 & tcatgtcattttaaccttg \\
\hline-3906 & -3888 & 1 & ttagggtttattgacctta \\
\hline & -3352 & -1 & aggattgacttgacccag \\
\hline $\begin{array}{l}-2569 \\
-1128\end{array}$ & -2551 & ${ }_{-1}^{-1}$ & ccagttaattgtgagcaaa \\
\hline $\begin{array}{l}-1128 \\
-1366\end{array}$ & . & ${ }_{1}^{-1}$ & ctggaacaccttgaccttt \\
\hline $\begin{array}{l}-1500 \\
-3077\end{array}$ & $\begin{array}{l}-1340 \\
-3059\end{array}$ & -1 & $\begin{array}{l}\text { aaaagataagatttgacctat } \\
\text { aaagattaatgtaacccraa }\end{array}$ \\
\hline-4761 & -4743 & -1 & tcatgatcatgtgacctac \\
\hline-404 & -386 & -1 & ataggcagtattgacctta \\
\hline 1784 & -1766 & 1 & agagatcaccatgacacaa \\
\hline-1603 & -1585 & -1 & tcttgccagtttgactaa \\
\hline 1784 & -1766 & 1 & agagatcaccatgacacaa \\
\hline-16 & -1585 & -1 & tcttgccagtttgacctaa \\
\hline-4961 & -4943 & -1 & caaagtgttctctgacccag \\
\hline-1168 & -1150 & -1 & acagtgaattctgaccaga \\
\hline & -172 & 1 & aaaggccagggtaacctaa \\
\hline $\begin{array}{l}-21844 \\
-2186\end{array}$ & -2456 & 1 & aacgcaaactctgacctaa \\
\hline $\begin{array}{l}-2150 \\
-2155\end{array}$ & -2168 & 1 & aacgcaaactctgacctaa \\
\hline & -427 & -1 & aacgcaaacttctgacctaa \\
\hline-2063 & -2045 & & ctggagcaccttgaccttc \\
\hline & -2045 & -1 & ctggagcaccttgaccttc \\
\hline-00 & -670 & 1 & gttaacatga \\
\hline & -813 & -1 & atcccctgtgaccttc \\
\hline-4078 & -4060 & -1 & tgcggtcaacttgaccatt \\
\hline 2977 & -2959 & -1 & caagtttatttgacctag \\
\hline & & 1 & tcaagtcattctgacttgc \\
\hline 1466 & -1448 & -1 & tccagcaactgtgaccaac \\
\hline & & -1 & tccagcaactgte \\
\hline $\begin{array}{c}-474 \\
-231\end{array}$ & $\begin{array}{l}-4729 \\
-2330\end{array}$ & 1 & $\begin{array}{l}\text { aaagacaaautttgaccttt } \\
\text { gccagatactotaagctac }\end{array}$ \\
\hline 2318 & -2300 & & gccagatactgtgagctgc \\
\hline-6 & -4446 & -1 & actggacartttgaccaggg \\
\hline & & & tcgtttcaatgtgacctga \\
\hline
\end{tabular}

